NON-LINEAR EQUATIONS OF KORTEWEG-DE VRIES TYPE, FINITE-ZONE LINEAR OPERATORS, AND ABELIAN VARIETIES

This article has been downloaded from IOPscience. Please scroll down to see the full text article. 1976 Russ. Math. Surv. 3159

(http://iopscience.iop.org/0036-0279/31/1/R03)

View the table of contents for this issue, or go to the journal homepage for more

Download details:

IP Address: 147.122.45.69

The article was downloaded on 31/01/2013 at 15:06

Please note that terms and conditions apply. 
Russian Math. Surveys 31:1 (1976), 59-146

From Uspekhi Mat. Nauk 31:1 (1976), 55-136

\title{
NON-LINEAR EQUATIONS OF KORTEWEG-DE VRIES TYPE, FINITE-ZONE LINEAR OPERATORS, AND ABELIAN VARIETIES
}

\author{
B. A. Dubrovin, V. B. Matveev, and S. P. Novikov
}

The basic content of this survey is an exposition of a recently developed method of constructing a broad class of periodic and almost-periodic solutions of non-linear equations of mathematical physics to which (in the rapidly decreasing case) the method of the inverse scattering problem is applicable. These solutions are such that the spectrum of their associated linear differential operators has a finite-zone structure. The set of linear operators with a given finite-zone spectrum is the Jacobian variety of a Riemann surface, which is determined by the structure of the spectrum. We give an explicit solution of the corresponding non-linear equations in the language of the theory of Abelian functions.

\section{Contents}

Introduction . . . . . . . . . . . . . . . . . . . . . . 60

Chapter 1. Examples of non-linear equations admitting a commutation representation. Methods of finding them . . . . . . . . . . . 66

$\S 1$. The $\mathrm{K}-\mathrm{dV}$ equation and its higher order analogues . . . . . 66

$\S 2$. The non-linear equation of the string and the two-dimensional $\mathrm{K}-\mathrm{dV}$ equation . . . . . . . . . . . . . . . . . . . 68

§3. The non-linear Schrödinger equation. . . . . . . . . . . 68

§4. First order matrix operators . . . . . . . . . . . . . . 69

$\S 5$. Discrete systems. The Toda chain and the "K-dV difference equation" . . . . . . . . . . . . . . . . . . 70

$\S 6$. The method of Zakharov and Shabat of constructing nonlinear equations that have an $L-A$ pair . . . . . . . . . 71

Chapter 2. The Schrödinger operator and the $k-d V$ equation. Finitezone potentials . . . . . . . . . . . . . . . . . 72

$\S 1$. General properties of the Schrödinger operator with a periodic and rapidly decreasing potential

§2. A new commutation representation of the $\mathrm{K}-\mathrm{dV}$ and "higher order $\mathrm{K}-\mathrm{dV}$ " equations. An algorithm for finding finite-zone potentials and their spectra . . . . . . . . . . . . .

$\S 3$. The inverse problem for periodic and almost-periodic (real and complex) finite-zone potentials. The connection with the theory of Abelian varieties. . . . . . . . . . . . . .

§4. Applications. The time dynamics of finite-zone potentials according to $\mathrm{K}-\mathrm{dV}$ equations. The universal fibering of Jacobian varieties (the hyperelliptic case). 
Chapter 3. Generalizations. Discrete systems and first order matrix operators .

$\S 1$. The periodic problem for the Toda chain and the "K- $\mathrm{dV}$ difference" equation . . . . . . . . . . . . . . 106

$\S 2$. First order matrix operators and their associated non-linear systems

Appendix 1. Non-reflective potentials against the background of finitezone potentials. Their algebraic-geometric axiomatics . . .

Appendix 2. Another method of obtaining some theorems in Ch.2, $\S 2$

Appendix 3. On the use of linear and non-linear trace formulae for the integration of equations of $\mathrm{K}-\mathrm{dV}$ type and the expression of the Bloch solution of the Schrödinger equation in terms of a $\theta$-function

Concluding Remarks

References

\section{Introduction}

In 1967 a remarkable mechanism was discovered relating some important non-linear wave equations with the spectral theory of auxiliary linear operators. This connection makes it possible, in a certain sense, to "integrate" these non-linear equations (see [18]). The first such equation, the famous Korteweg-de Vries (K-dV) equation, was reduced in [18] to the inverse scattering problem for the Schrödinger operator $L=-d^{2} / d x^{2}+u(x)$, to solve the Cauchy problem for the K-dV equation in the class of rapidly decreasing functions $u(x)$. Subsequently, this mechanism was perfected and interpreted from different points of view by Lax [19], Zakharov and Faddeev [21], and Gardner [22]; later, other important non-linear equations were found to which a similar mechanism can also be applied. The first after the K-dV equation was the non-linear Schrödinger equation (Zakharov and Shabat [25], [26]) then the standard sine-Gordon equation, the Toda chain, the non-linear equation of the string, and a number of others (see [23]-[35]); for all these equations an analogue of the method of Gardner-Green-Kruskal-Miura makes it possible to "integrate" the Cauchy problem for rapidly decreasing functions of $x$, by means of the scattering theory for an auxiliary linear operator. In particular, this method enables us to investigate the asymptotic behaviour of solutions in time and to obtain some important particular solutions called "multisoliton", which describe the interaction of a finite number of "solitons" (isolated waves of the form $u(x-c t))$.

Up to the end of 1973 there were no papers in which this mechanism was used successfully to study the Cauchy problem for functions periodic in $x$, even for the original $\mathrm{K}-\mathrm{dV}$ equation. This is not surprising: one of 
the reasons is that the inverse problem for the Schrödinger equation with periodic potential was, in essence, unsolved. There were no effective methods whatever of finding the potential from the spectral data. (For almost periodic potentials this problem had not even been raised.) Recently, Novikov, Dubrovin, Matveev and Its have published a number of papers (see [38]-[43], [46], [47]) in which a method is developed that permits us to find a broad set of exact solutions of the K-dV equation, periodic and almost periodic in $x$, which give a natural generalization of the multisoliton solutions. It is fairly clear that this set of solutions is dense among the periodic functions. This was noted in [42] and [43], but has not yet been established rigorously. The method of the present authors, which is expounded in this survey, required a substantial perfection of the algebraic mechanism mentioned above, and also an appeal to ideas of algebraic geometry. This method is applicable not only to the $\mathrm{K}-\mathrm{dV}$ equation, but also to other non-linear equations of this type, for the investigation of the periodic problem, as was pointed out in [42] and [43]. The appropriate modifications of the method will be indicated in the survey.

At the International Congress of Mathematicians in Vancouver it became known that simultaneously with Novikov's first paper [38], the work of Lax [50] appeared, which contains as its principal result part of the main theorem of Novikov's paper [38]; Lax's proof is not constructive and differs from Novikov's method (see Ch. 2, $\$ 2$, p. 84). Furthermore, Marchenko has completed his works [44] and [45] in which he develops a method of successive approximations to solutions of the K-dV equation, which is based on the spectral theory of the Schrödinger operator $L$. Some of his arguments overlap with specific technical arguments of [38].

In studying a periodic or almost-periodic problem by the method of the authors an important part is played by the class of potentials $u(x)$ for which the Schrödinger operator $L=-d^{2} / d x^{2}+u(x)$ has only finitely many forbidden zones (lacunae or zones of instability) in the spectrum on the whole real $x$-axis.

We must bear in mind that the physical interpretation of the K-dV equation in the theory of non-linear waves (see [16]) is such that its natural Cauchy problem must be posed without definite boundary conditions (for example, periodic functions with a given period in $x$ are insufficient). We must find as wide as possible a set of solutions $u(x, t)$ belonging to various classes of functions that are bounded in $x$. The most natural such classes are, apart from the class of rapidly decreasing functions already mentioned, those of periodic functions with arbitrary periods in $x$, and also those of almost periodic functions of $x$ with an arbitrary group of periods (in any case, the dynamics in $t$ turns out to be almost-periodic a posteriori).

The formulation in which we shall solve the inverse problem for the Schrödinger operator $L$ automatically gives not only periodic, but also almost-periodic real and complex potentials and the corresponding solutions 
of the K-dV equation. Our approach, based on the consideration of finitezone potentials, is closely connected both with the theory of the K-dV equation and with the algebraic geometry of Riemann surfaces and Abelian varieties (see Ch. 2). This is a constructive approach and enables us to obtain exact analytic solutions. It is noteworthy that the connection with the $\mathrm{K}-\mathrm{dV}$ equation gives non-trivial new results in the theory of Abelian varieties itself, in particular, it leads to explicit formulae for the universal fibering of the Jacobian varieties of hyperelliptic Riemann surfaces (for example, even the fact that this covering space is unirational was not known hitherto).

Examples of finite-zone potentials (but in a context unrelated to algebraic geometry and the $\mathrm{K}-\mathrm{dV}$ equation) have in the past aroused the interest of various mathematicians in isolation, beginning with Ince who noted in 1940 that the potential of the Lamé problem, which coincides with the doubly elliptic function of Weierstrass, has only one lacuna, but the $n(n+1) / 2-$ fold Lamé potentials have $n$ lacunae (see [7]). In 1961, Akhiezer [10] began to construct examples of finite-zone potentials on the half-line $x \geqslant 0$ without knowing of Ince's work [7]. He proposed an interesting (in essence, algebraicgeometric) method of constructing the eigenfunctions without constructing the potentials themselves. The potentials which result from Akhiezer's construction, turn out under analytic continuation in $x$ to be even almostperiodic functions of $x$; true, he paid no attention to this and considered the whole problem only on the half-line and pursued the analysis to its conclusion only in the single-zone case. The development of Akhiezer's ideas, which was undertaken by Dubrovin [40] and Matveev and Its [41], plays a large part in the method of the present authors (see Ch. 2).

In 1965, Hochstadt [9], without knowing of Akhiezer's work [10], raised the inverse problem for finite-zone periodic potentials and began to solve it. He proved that there are no potentials other than elliptic functions with a single lacuna (for $n=1$ this theorem is the converse of the result of Ince). For a number $n>1$ of lacunae Hochstadt could only prove that a continuous $n$-lacuna potential is an infinitely smooth function. Such is the history of this problem prior to the recent papers covered in this survey.

We are particularly concerned with the properties of the one-dimensional Schrödinger (Sturm-Liouville) equation with an almost-periodic potential $u(x)$. Before the publication of [38] there were in the literature neither serious general results, nor integrable cases of this problem. In fact, the translation matrix $\hat{T}$, the law of dispersion $p(E)$, and the Bloch eigenfunctions $\psi_{ \pm}$are formally meaningless. Although the Bloch eigenfunction $\psi_{ \pm}\left(x, x_{0}, E\right)$ can here also be defined by requiring that the function

$$
\chi(x, E)=\frac{d \ln \dot{\psi}_{ \pm}}{d x}
$$


has the same group of periods as the potential (instead of the condition

$$
\left.\psi_{ \pm}(x+T)=e^{ \pm i p(E)} \psi_{ \pm}(x)\right),
$$

it is no longer clear whether it exists for all real and complex values of the spectral parameter $E, L \psi_{ \pm}=E \psi_{ \pm}$(the strongest results on this problem were obtained quite recently in [59]); the usual spectrum of the operator $L$ in $\mathscr{L}_{2}(-\infty, \infty)$ is obtained when $\psi_{ \pm}$is bounded, but we need it for all complex values of $E$. We shall study the class of real and complex almostperiodic potentials $u(x)$ that possess what we call correct analytic properties: for these an analogue of the Bloch eigenfunction $\psi_{ \pm}\left(x, x_{0}, E\right)$ exists for all complex $E$ and it has the following properties (see Ch. 2, $\S 2)$ :

1) $L \psi_{ \pm}=E \psi_{ \pm}$

2) $\left.\psi_{ \pm}\left(x, x_{0}, E\right)\right|_{x=x_{0}} \equiv 1$

3) $\psi_{ \pm} \sim e^{ \pm i k\left(x-x_{0}\right)}$ as $E \rightarrow \infty, k^{2}=E$;

4) $\psi_{ \pm}=\frac{d \ln \psi_{ \pm}}{d x}$ is almost periodic with the same group of periods as the potential $u(x)$;

5) $\psi_{ \pm}\left(x, x_{0}, E\right)$ is meromorphic on a Riemann surface $\Gamma$ doubly covering the $E$-plane and $\psi_{ \pm}$and $\psi_{-}$are obtained from one another by exchanging the two sheets.

It is permitted here also to consider potentials with poles at certain points; in this case, if the potential is unbounded, we assume the function $u(x)$ to be complex analytic (meromorphic) in a certain strip $x+i y$ around the $x$-axis.

We say that the potential $u(x)$ (or the operator $L$ ) is finite-zoned (finitely lacunary) if the Riemann surface $\Gamma$ has finite genus. $\Gamma$ itself is called the spectrum of the operator $L$, its genus is the number of forbidden zones (or lacunae), and the branch-points are "boundaries of the zones" (or lacunae), although a direct spectral interpretation of these concepts is valid only in the case of bounded real potentials $u(x)$.

Novikov in [38] observed that it is natural to consider periodic potentials in the solution of inverse scattering problems. He proved there that every stationary periodic solution $u(x)$ of the so-called "higher order $\mathrm{K}-\mathrm{dV}$ equations" is a finite-zone potential and that these stationary K-dV equations are themselves totally integrable Hamiltonian systems with $n$ degrees of freedom ( $n$ being the number of zones). Hence their general solution is an almost-periodic function, possibly meromorphic. These equations depend on $n+1$ constants $c_{0}, \ldots, c_{n}$ and have $n$ commuting integrals $J_{1}, \ldots, J_{n}$; furthermore, symmetric functions of the boundaries of the forbidden zones (lacunae) can be expressed in terms of these constants, and the levels of the integrals $\left(c_{0}, \ldots, c_{n}, J_{1}, \ldots, J_{n}\right)$. From this there follows an important result: for any given boundaries of zones (lacunae) we can find a potential 
by solving stationary higher $K-d V$ equations, but, generally speaking, it is almost-periodic with group of periods $T_{1}, \ldots, T_{n}$ and may possibly have poles. All the right-hand sides of the $K-d V$ equations and of the integrals $J_{\alpha}$ are polynomials, and naturally there arise also complex meromorphic almost-periodic potentials.

Soon after the completion of the papers of Dubrovin [40] and Its and Matveev [41], Novikov and Dubrovin showed [43] that the complex level surface of the integrals $J_{1}, \ldots, J_{n}$ is an Abelian variety, explicitly embedded in projective space; all the potentials $u(x)$ have regular analytic properties and are almost-periodic (if $x$ is complex) with a group of $2 n$ "real and imaginary" periods $T_{1}, \ldots, T_{n}, T_{1}^{\prime}, \ldots, T_{n}^{\prime}$, and their time evolution by the $\mathrm{K}-\mathrm{dV}$ equation and all its higher order analogues for these potentials is given by a rectilinear (complex) development of this Abelian variety (the $2 n$-dimensional complex torus). This Abelian variety is called the "Jacobian variety" of $\Gamma$.

These results can also be applied directly to the theory of Abelian varieties (see Ch. 2). To see this it suffices to note that in the solution of the inverse problem of the spectral theory for the Schrödinger operator $L$ by the method of the present authors all reference to results from the theory of the Schrödinger operator with periodic potential is easily eliminated, and all the results are true for the almost-periodic case when the boundaries of the zone (branch-points of $\Gamma$ ) are given arbitrarily. The group of periods of $u(x)$ is determined by the spectrum (by $\Gamma$ ); to specify the potential $u(x)$ we need one arbitrary point on the complex torus - the Jacobian variety $\mathbf{J}(\Gamma)$. Explicit formulae are given for the potential $u(x)$. There are formulae of several types (see Ch. 2); the best of them seems to be that due to Matveev and Its (see [41] and Ch. 2, \$3): it expresses the potential by a simple explicit formula in terms of the Riemann $\theta$-function. A number of useful formulae for the time dynamics according to $\mathrm{K}-\mathrm{dV}$ can be found in [46]. We recall that in the usual statement of the inverse problem for a periodic potential as "scattering data" all the eigenvalues $\left(E_{n}\right)$ are given for which the eigenfunctions are periodic with the same period $T$ as the potential, as well as the residual spectrum of the Sturm-Liouville problem on the half-line (see, for example, [6]; uniqueness theorems were already proved by Borg [1]). In this form even finite-zoned potentials cannot be effectively distinguished.

In our formulation (for example, for the finite-zone case) the boundaries of the zones are specified arbitrarily, then all the potentials with this spectrum which have a common group of periods and form the Jacobian variety $\mathbf{J}(\Gamma)$ are found explicitly. If the branch-points (boundaries of zones) are all real and the poles of the function $\psi_{ \pm}\left(x, x_{0}, E\right)$ lie at points of the "spectrum" $\Gamma$ over finite lacunae, then we obtain a family of bounded almost-periodic real potentials, forming a real torus $T^{n}$, on which the higher equations of $\mathrm{K}-\mathrm{dV}$ type form rectilinear developments in the corresponding 
"angular" variables (see [46]).

If even the potential $u(x)$ obtained is real and periodic in $x$, then under continuation into the complex domain in $x$, it becomes conditionally periodic along the imaginary axis, generally speaking, with a group of $n$ imaginary periods $T_{1}^{\prime}, \ldots, T_{n}^{\prime}$. The only exceptions are the cases when the potential reduces to elliptic functions. Such potentials are given, for example, in Ch. 2 and in [42]. The simplest is the Lamé potential.

It is not difficult to obtain the "closure" of our formulations, as the number of zones tends to infinity. However, in this case the relevant theorems become "existence and uniqueness theorems" and cease to be analytically constructive. It has not yet been proved rigorously that any (periodic) potential can be approximated by finite-zone potentials.

In Ch. 3 we give generalizations of our theorems to some other nonlinear equations. As was pointed out to the authors by A. N. Tyurin, of particular interest from the point of view of algebraic geometry is the translation of the theory to the case of first order $(n \times n)$-matrix operators with $n>2$, in which non-hyperelliptic Riemann surfaces appear. If arbitrary Riemann surfaces can, in fact, occur in the periodic theory of ZakharovShabat operators (see Ch. 3, §2) as "spectra" of a finite-zone linear operator, then this leads to a proof of the celebrated conjecture that the space of moduli of Riemann surfaces of arbitrary genus is unirational. Indeed, according to the scheme of [43] (see $\mathrm{Ch}$. 2, §3) we can show that the space of the universal fibering of Jacobian varieties is unirational. But then the base also (that is, the variety of moduli of the curves themselves) is also unirational. However, the question of whether all Riemann surfaces $\Gamma$ can occur in this way, is not yet settled.

Thus, in naturally occurring totally Liouville integrable Hamiltonian systems connected with the scattering theory for the auxiliary local operator $L$, the level surfaces of the commuting integrals are not simply real tori $T^{n}$, but under continuation into the complex domain, they are Abelian varieties $T^{2 n}$ which are the Jacobian varieties $\mathbf{J}(\Gamma)$ of Riemann surfaces arising as spectra of the operator $L$. An interesting question is whether there are natural classes of totally integrable systems in which there arise similarly Abelian varieties that are not the Jacobian varieties of any Riemann surfaces.

Incidentally, as a concluding remark we point out that in the classical nontrivial integrable cases of Jacobi (geodesics on a 3-dimensional ellipsoid) and of Sonya Kovalevski (the case of the heavy gyroscope) Abelian varieties also occur as level surfaces of commuting integrals: the direct product of onedimensional ones for Jacobi's case and non-trivial two-dimensional Abelian varieties for the Kovalevski case. (This can be guessed from the formulae given, for example, in [57], although the result has not been stated anywhere explicitly.) Here we quote from a letter written by Sonya Kovalevski in December 1886. 
"He (Picard) reacted with great disbelief when I told him that functions of the form

$$
y=\frac{\theta\left(c x+a, c_{1} x+a_{1}\right)}{\theta_{1}\left(c x+a, c_{1} x+a_{1}\right)}
$$

can be useful in the integration of certain differential equations" (quoted in [57]). The analysis of the present authors shows that for 90 years after the work of Sonya Kovalevski, until the 1974 papers on the K-dV equations, Picard's scepticism was justified.

The present survey consists of three chapters and applications. The first chapter is a brief survey of the non-linear equations integrable by the method of the inverse scattering problem that were known up to the end of 1974. The second and third chapters and the Appendices contain an account of the results of the authors, Its and Krichever.

\section{CHAPTER 1 \\ EXAMPLES OF NON-LINEAR EQUATIONS ADMITTING A COMMUTATION REPRESENTATION. METHODS OF FINDING THEM}

$\S 1$. The K-dV equation and its higher order analogues

As Lax pointed out in 1968 (see [19]), the Kruskal-Gardner-GreenMiura method [18] of integrating the Cauchy problem for functions rapidly decreasing in $x$ for the $\mathrm{K}-\mathrm{dV}$ equation $u_{t}=6 u u_{x}-u_{x x x}$ can be obtained, by reduction to the inverse scattering problem for the Schrödinger operator, from the operator representation of this equation

$$
\frac{d L}{d t}=[A, L]
$$

in which $L=-\frac{d^{2}}{d x^{2}}+u(x, t)$ and $A=-4 \frac{d^{3}}{d x^{3}}+3\left(u \frac{d}{d x}+\frac{d}{d x} u\right)$. The operators $L$ and $A$ act on functions on $x, d L / d t$ is the operator of multiplication by the function $u_{t}$, while $[A, L]$ is the operator of multiplication by $6 u u_{x}-u_{x x x}$. It follows from (1.1.1) that the discrete spectrum $\lambda_{i}(L)$ does not change in time. This is true both for functions $u(x, t)$ rapidly decreasing in $x$ and also for periodic functions (although in the latter case this gives a set of integrals equivalent to the Kruskal-Zabusskii integrals [17]. Lax and Gardner (see [19], [22]) indicated a series of evolution equations of the form

$$
u_{t}=Q_{n}\left(u, \frac{\partial u}{\partial x}, \frac{\partial^{2} u}{\partial x^{2}}, \ldots, \frac{\partial^{2 n+1} u}{\partial x^{2 n+1}}\right)
$$

admitting an analogous representation

$$
\frac{d L}{d t}=\left[A_{n}, L\right]
$$


where $A_{n}=\frac{d^{2 n+1}}{d x^{2 n+1}}+\sum_{i=0}^{2 n} P_{i} \frac{d^{i}}{d x^{i}}, P_{i}$ and $Q_{n}$ are polynomials in $u, u_{x}, u_{x x x}, \ldots$ with constant coefficients, $L$ is the Schrödinger operator $L=-\frac{d^{2}}{d x^{2}}+u(x, t)$, $A_{0}=\frac{d}{d x}, A_{1}=A$, and

$$
A_{2}=16 \frac{d^{5}}{d x^{5}}-20\left(u \frac{d^{3}}{d x^{3}}+\frac{d^{3}}{d x^{3}} u\right)+30 u \frac{d}{d x} u+5\left(u^{\prime \prime} \frac{d}{d x}+\frac{d}{d x} u^{\prime \prime}\right) .
$$

All these are called "higher order K-dV equations" and have the form $Q_{n}=\frac{\partial}{\partial x} \frac{\delta I_{n}}{\delta u(x)}$, where $I_{n-1}=\int \chi_{2 n+1}\left(u, u_{x}, \ldots, u^{(n-1)} d x\right.$ and the $\chi_{2 n+1}$ are polynomials to be specified below; for example,

$$
\left\{\begin{array}{l}
I_{-1}=\int u d x, \quad I_{0}=\int u^{2} d x, \quad I_{1}=\int\left(\frac{u_{x}^{2}}{2}+u^{3}\right) d x, \\
I_{2}=\int\left(\frac{1}{2} u_{x x}^{2}-\frac{5}{2} u^{2} u_{x x}+\frac{5}{2} u^{4}\right) d x .
\end{array}\right.
$$

As was shown first in [17], the quantities $I_{n}$ are conserved in time by the K-dV equation. From the representation $Q_{n}=\frac{\partial}{\partial x} \frac{\delta I_{n}}{\delta u(x)}$ it follows, as Gardner noted, that all the "higher order K-dV equations" are Hamiltonian systems with infinitely many degrees of freedom, since $\frac{\partial}{\partial x}$ is a skewsymmetric operator. Faddeev and Zakharov [21] have shown that all the $\mathrm{K}-\mathrm{dV}$ equations are totally integrable Hamiltonian systems in which the scattering data of $L$ (see Ch. $2, \S 1$ ) are "action-angle" canonical variables, and the eigenvalues of the discrete spectrum $\lambda_{i}(L)$ commute (have vanishing Poisson brackets). From this it follows that the integrals $I_{n}$ also commute. This last fact was proved independently by Gardner [22] by another method. An algorithm for discovering the integrals $I_{n}$ is as follows (these integrals were first found in 1965). Let $L \psi=E \psi$ and $i \chi(x, k)=\frac{d \ln \psi}{d x}$, where $k^{2}=E$. Then the quantity $\chi$ satisfies the Riccati equation

$$
-i \chi^{\prime}+\chi^{2}+u-E=0
$$

and admits, by (1.1.4), the formal expansion as $E \rightarrow \infty$ :

$$
\chi(x, k) \sim k+\sum_{n=1}^{\infty} \frac{\chi_{n}(x)}{(2 k)^{n}}, \quad k^{2}=E .
$$

All the polynomials $\chi_{2 n}(x)$ are purely imaginary and are total derivatives, while the polynomials $\chi_{2 n+1}(x)$ are real and depend on $u, u_{x}, u_{x x}, \ldots, u^{(n-1)}$. 
For all $k$, the integral $I_{k}=\int_{-\infty}^{\infty} \chi(x, k) d x$ is time-invariant under all "higher order K-dV equations", hence, all the quantities $I_{n}$ are also conserved. The scattering data and also the method of integrating the periodic problem will be explained later (see $\mathrm{Ch}$. 2). We know that the modified $\mathrm{K}-\mathrm{dV}$ equation $u_{t}=6|u|^{2} u_{x}-u_{x x x}$ can be reduced to the $\mathrm{K}-\mathrm{dV}$ equation, hence is also totally integrable.

\section{§2. The nom-linear equation of the string and the two dimensional K-dV equation}

Closest to the $\mathrm{K}-\mathrm{dV}$ equation from the present point of view are the nonlinear equations of the string (see [23])

$$
\frac{3}{4} \beta \frac{\partial u}{\partial y}=-\frac{\partial w}{\partial x}, \quad \beta \frac{\partial w}{\partial y}=\frac{1}{4}\left(6 u u_{x}+u_{x x x}\right)+\lambda u_{x}
$$

and the "two-dimensional K-dV equation" (see [24], [37])

$$
\left\{\begin{aligned}
\frac{3}{4} \beta \frac{\partial u}{\partial y} & =-\frac{\partial w}{\partial x} \\
\beta \frac{\partial w}{\partial y} & =\alpha \frac{\partial u}{\partial t}+\lambda u_{x}+\frac{1}{4}\left(6 u u_{x}+u_{x x x}\right)
\end{aligned}\right.
$$

which occurred earlier in the study of transverse perturbations of long waves in non-linear media with dispersion. Here the operators $L$ and $A$ have the form

$$
L=\frac{d^{2}}{d x^{2}}+u, \quad A=4 \frac{d^{3}}{d x^{3}}+3\left(u \frac{d}{d x}+\frac{d}{d x} u\right)+\lambda \frac{d}{d x}+w .
$$

The equation (1.2.1) is equivalent to the following:

$$
\beta \frac{\partial A}{\partial y}=[L, A] .
$$

and (1.2.2) to the relation

$$
\beta \frac{\partial A}{\partial y}+\alpha \frac{\partial L}{\partial t}=[L, A] .
$$

In fact, in (1.2.1) and (1.2.3) the operators $L$ and $A$ have exchanged their roles in comparison with the K-dV equation, and the two-dimensional $\mathrm{K}-\mathrm{dV}$ equation ((1.2.2) and (1.2.4)) unites them.

\section{§3. The non-linear Schrödinger equation}

This equation has the form

$$
\alpha^{\prime} \frac{\partial u}{\partial t}=\frac{\partial^{2} u}{\partial x^{2}} \pm|u|^{2} u
$$

and was after the K-dV equation the first for which Zakharov and Shabat discovered in 1970 a mechanism for the reduction to the scattering problem for the auxiliary linear operator, which is no longer a Schrödinger operator 
(see [25], [26]). Here the operators $L$ and $A$ have the form

$$
\begin{gathered}
L=I \frac{d}{d x}+\left(\begin{array}{ll}
0 & u \\
v & 0
\end{array}\right)\left(l_{1}-l_{2}\right), \quad I=\left(\begin{array}{ll}
l_{1} & 0 \\
0 & l_{2}
\end{array}\right), \\
A=\frac{d^{2}}{d x^{2}}+2\left(\begin{array}{ll}
0 & u_{x} \\
v_{x} & 0
\end{array}\right) .
\end{gathered}
$$

If $u=\vec{v}$, then the equation

$$
\alpha \frac{\tau L}{d t}=[A, L]
$$

is equivalent to $(1.3 .1)$ :

$$
-\alpha \frac{\partial u}{\partial t}=\frac{l_{1}+l_{2}}{l_{2}-l_{1}} u_{x x}-2 \frac{l_{1}+l_{2}}{l_{1} l_{2}}|u|^{2} u
$$

for an appropriate choice of constants $l_{1}$ and $l_{2}$. The integration of (1.3.1) for rapidly decreasing functions can be found in [25] and [26].

\section{§4. First order matrix operators}

Suppose that $L$ and $A$ are first order matrix operators in $x$. We look for them in the form

$$
L=l_{1} \frac{d}{d x}+\left[l_{1}, \xi\right], \quad A=l_{2} \frac{d}{d x}+\left[l_{2}, \xi\right],
$$

where $\left[l_{1}, l_{2}\right]=0, l_{1}, l_{2}$ are constant $(N \times N)$-matrices and $\xi=\left(\xi_{i j}\right)$. The matrices $l_{1}$ and $l_{2}$ can be assumed to be diagonal, $l_{1}=a_{i} \delta_{i j}, l_{2}=b_{i} \delta_{i j}$. The equation

$$
\alpha \frac{\partial L}{\partial t}+\beta \frac{\partial A}{\partial y}=[L, A]
$$

can easily be brought to the form

$$
\left(a_{i}-a_{j}\right) \frac{\partial \xi_{i j}}{\partial t}=\left(b_{i}-b_{j}\right) \frac{\partial \xi_{i j}}{\partial y}+\ldots
$$

In a number of cases, by imposing on $\xi_{i j}$ constraints of the type $\xi^{+}=I \xi I$, where $I^{2}=1$, we can reduce (1.4.2) to a system with fewer unknowns. Some important cases of this kind for $N=3$ were first given in [27], with $I=\left(\begin{array}{lll}1 & 1 & 0 \\ 0 & 1 & 1\end{array}\right)$ or $I=\left(\begin{array}{lll}1 & -1 & 0 \\ 0 & 1 & 1\end{array}\right)$. A general method of obtaining these systems was developed in [37].

The degenerate case in which some of the components of the matrices $l_{1}, l_{2}$ vanish, $a_{i}=b_{i}=0$, is very interesting. For fourth order matrices we have here systems for which the corresponding non-linear equation reduces to the standard "sine-Gordon" equation (see [30]):

$$
u_{t t}-u_{x x}=\sin u \text {. }
$$

As a consequence of the degeneracy $a_{i}=b_{i}$ mentioned above, the spectral problem for the corresponding operator $L$ turns out to be nontrivial; various difficulties are overcome in [30]. More straightforward is the 
variant of the "sine-Gordon" equation

$$
u_{\xi \eta}=-\sin u \text {, }
$$

in which the initial data are placed on a single characteristic. This case is simpler. It was studied previously in [29] and [28]. Here the spectral problem is not degenerate, and the operators $L, A$ for $(1.4 .4)$ have the form

$$
L=\left(\begin{array}{rr}
0 & -1 \\
1 & 0
\end{array}\right) \frac{d}{d x}+\frac{i}{2} u_{\xi}\left(\begin{array}{ll}
0 & 1 \\
1 & 0
\end{array}\right)
$$

$A \psi(x)=$

$$
=\frac{1}{4} \int_{-\infty}^{x}\left(\begin{array}{cc}
\exp \left(-\frac{i}{2}\left(u(x, t)+u\left(x^{\prime}, t\right)\right)\right) & 0 \\
0 & \exp \left(\frac{i}{2}\left(u(x, t)+u\left(x^{\prime}, t\right)\right)\right)
\end{array}\right) \psi\left(x^{\prime}\right) d x^{\prime} .
$$

\section{$\S 5$. Discrete systems}

The Toda chain and the "K-dV equation difference"

We consider a chain of particles in a straight line with coordinates $x_{n}$ and the interaction Hamiltonian $H=\sum_{n}\left(e^{x_{n}-x_{n-1}}+\dot{x}_{n}^{2} / 2\right)$, which was first discussed in [31]. The integrals found by Henon in [32] for the Toda chain explicitly showed its integrability. In [33]-[35], the $L-A$ pair for this chain was found and the commutativity of the integrals previously found by Henon was proved. For the "rapidly decreasing" case, where $c_{n} \rightarrow 1, v_{n} \rightarrow 0\left(c_{n}=e^{x_{n}-x_{n-1}}, v_{n}=\dot{x}_{n}\right)$, the Toda chain was integrated by the scattering theory method. Here the equations have the form

$$
\dot{v}_{n}=c_{n+1}-c_{n}, \quad \dot{c}_{n}=c_{n}\left(v_{n}-v_{n-1}\right)
$$

and the operators $L$ and $A$ are as follows:

$$
\left\{\begin{array}{l}
L_{m n}=i \sqrt{c_{n}} \delta_{n, m_{+1}}-i \sqrt{c_{m}} \delta_{n+1}, m+v_{n} \delta_{m n}, \\
A_{m n}=\frac{i}{2}\left(\sqrt{c_{n}} \delta_{n, m_{+1}}+\sqrt{c_{m}} \delta_{n+1}, m\right) .
\end{array}\right.
$$

(1.5.1) is equivalent to the equation $d L / d t=[A, L]$.

In addition to the Toda chain (1.5.1), in [35] another " $\mathrm{K}-\mathrm{dV}$ difference equation" is considered:

$$
\dot{c}_{n}=c_{n}\left(c_{n+1}-c_{n-1}\right) \text {, }
$$

which can be obtained from the $L-A$ pair (1.5.2) with the same operator $L$ under the condition $v_{n} \equiv 0$ and the new operator $A \rightarrow \bar{A}$, where

$$
\bar{A}=-\frac{1}{2}\left(\sqrt{c_{n} c_{n-1}} \delta_{n, m_{+2}}-\sqrt{c_{m} c_{m-1}} \delta_{n+2, m}\right)
$$

then $(1.5 .3)$ is equivalent to the equation $d L / d t=[\bar{A}, L], v_{n} \equiv 0$. The 
operator $L$ itself, given by (1.5.2), is a difference analogue to a SturmLiouville operator and was considered previously for other purposes [12], where the inverse problem on the half-line $n \geqslant 0$ was solved for it.

\section{$\S 6$. The method of Zakharov and Shabat of constructing non-linear equations that have an $L-A$ pair}

Let $\hat{F}$ be a linear integral operator acting on the vector function $\left(\psi_{1}, \ldots, \psi_{N}\right)=\psi$ of the variable $x(-\infty<x<\infty)$ :

$$
\hat{F} \psi=\int_{-\infty}^{\infty} F(x, z) \psi(z) d z
$$

where $\psi$ and $\hat{F}$ depend in addition on two parameters $t$ and $y$. Let us assume that the operator $\hat{F}$ admits the following representation:

$$
1+\hat{F}=\left(1+K_{+}\right)^{-1}\left(1+K_{-}\right),
$$

in which $K_{+}$and $K_{-}$are Volterra integral operators with

$$
\left\{\begin{array}{l}
K_{+}(x, z)=0, \quad z<x \\
K_{-}(x, z)=0, \quad z>x
\end{array}\right.
$$

From (1.6.1) and (1.6.2) there follows the (Gel'fand-Levitan) equation for the kernel $K_{+}$:

$$
F(x, z)+K_{+}(x, z)+\int_{x}^{\infty} K_{+}(x, s) F(s, z) d s=0,
$$

and the kernel $K_{-}$can be found from the formula

$$
K_{-}(x, z)=F(x, z)+\int_{x}^{\infty} K_{+}(x, s) F(s, z) d s .
$$

We consider the operator $M_{0}=\alpha \frac{\partial}{\partial t}+\beta \frac{\partial}{\partial y}+L_{0}$, acting on $\psi(t, y, x)$, where $L_{0}=\sum_{n} l_{n} \partial^{n} / \partial x^{n}$, and the $l_{n}$ are constant $(N \times N)$-matrices. By analogy with the theory of the inverse scattering problems we can establish the following fact: if the operators $M_{0}$ and $\hat{F}$ commute, $\left[M_{0}, \hat{F}\right]=0$, then the transformed operator $M=\left(1+K_{+}\right) M_{0}\left(1+K_{+}\right)^{-1}$ is also a differential operator with variable coefficients

$$
M=\left(1+K_{+}\right) M_{0}\left(1+K_{+}\right)^{-1}=\alpha \frac{\partial}{\partial t}+\beta \frac{\partial}{\partial y}+L,
$$

where $L$ is an operator involving only differentials in $x$. In scattering theory this fact was known for the Shrödinger operator and was used for the operator $L_{0}=\frac{d^{2}}{d x^{2}}$.

We assume now that $M_{\delta}^{(1)}$ and $M_{0}^{(2)}$ are two operators such that 


$$
\begin{cases}M_{0}^{(1)}=\alpha \frac{\partial}{\partial t}+L_{0}^{(1)}, & L_{0}^{(1)}=\sum l_{n}^{(1)} \frac{\partial^{n}}{\partial x^{n}}, \\ M_{0}^{(2)}=\beta \frac{\partial}{\partial y}+L_{0}^{(2)}, & L_{0}^{(2)}=\sum l_{n}^{(2)} \frac{\partial^{n}}{\partial x^{n}}\end{cases}
$$

and $\left[M_{0}^{(1)}, M_{0}^{(2)}\right]=0$.

If $\hat{F}$ commutes with both of these, $\left[\hat{F}, M_{0}^{(1)}\right]=\left[\hat{F}, M_{0}^{(2)}\right]=0$, then we obtain the differential operators

$$
\left\{\begin{array}{c}
M^{(1)}=\left(1+K_{+}\right) M_{0}^{(1)}\left(1+K_{+}\right)^{-1}=\alpha \frac{\partial}{\partial t}+L^{(1)}, \\
M^{(2)}=\left(1+K_{+}\right) M_{0}^{(2)}\left(1+K_{+}\right)^{-1}=\beta \frac{\partial}{\partial y}+L^{(2)}, \\
{\left[M^{(1)}, M^{(2)}\right]=0 .}
\end{array}\right.
$$

The relations (1.6.7) lead to an equation for the operators $L^{(1)}$ and $L^{(2)}$ :

$$
\alpha \frac{\partial L^{(2)}}{\partial t}-\beta \frac{\partial L^{(1)}}{\partial y}=\left[L^{(2)}, L^{(1)}\right],
$$

which is equivalent to a system of non-linear equations for the coefficients of $L^{(1)}$ and $L^{(2)}$. Here the kernel $F(x, z, t, y)$ of $\hat{F}$ satisfies a system of linear equations with constant coefficients, which follow from the identities

$$
\left[\hat{F}, M_{0}^{(1)}\right]=\left[\hat{F}, M_{0}^{(2)}\right]=0 .
$$

We can easily solve the Cauchy problem in time $t$ for the equations (1.6.9) in the usual way, and then, having solved the Gel'fand-Levitan equation (1.6.4) define the coefficients of the operators $L^{(1)}$ and $L^{(2)}$ at any time $t$. In principle, this procedure can lead to the integration of the Cauchy problem for (1.6.8) only for functions rapidly decreasing in $x$ as coefficients of $L^{(1)}$ and $L^{(2)}$. We note that the method of ZakharovShabat permits us to construct a number of new systems with an $L-A$ pair, among them some of physical interest (see [371); this method both constructs the systems and produces a way of solving the inverse problem the Gel'fand-Levitan equation. Some of the above examples of $L-A$-pairs were first found in this manner.

\section{CHAPTER 2}

\section{THE SCHRÖDINGER OPERATOR AND THE K-dV EQUATION. FINITE-ZONE POTENTIALS}

\section{§1. General properties of the Schrödinger operator with a} periodic and rapidly decreasing potential

We first consider the usual Schrödinger operator $L=-\frac{d^{2}}{d x^{2}}+u$ from the requisite point of view. It is convenient to fix a basis in the space of solu- 
tions of the equation $L \varphi=E \varphi$. Let $x_{0}$ be a given point. We specify a solution $\varphi\left(x, x_{0}, \pm k\right)$ by setting

$$
\left\{\begin{array}{l}
\text { a) } \quad L \varphi\left(x, x_{0}, k\right)=k^{2} \varphi\left(x, x_{0}, k\right) \\
\text { b) } \varphi\left(x_{0}, x_{0}, k\right)=1, \\
\text { c) } \quad \varphi^{\prime}\left(x, x_{0}, k\right)=i k \text { when } x=x_{0}\left(k^{2}=E\right) .
\end{array}\right.
$$

Then we have a basis $\varphi(k), \varphi(-k)$ for all $k \neq 0$; for real $k$ (or $E>0$ ) we have $\varphi(-k)=\varphi(k)$.

We obtain another basis $c\left(x, x_{0}, E\right), s\left(x, x_{0}, E\right)$ as follows:

$$
\left\{\begin{array}{ll}
c=1, & c^{\prime}=0, \\
s=0, & s^{\prime}=1
\end{array} \text { when } x=x_{0} .\right.
$$

If the potential $u(x)$ is periodic with period $T$, then the operator of translation (or "monodromy") is defined by

$$
(\hat{T} \psi)(x)=\psi(x+T) .
$$

The translation operator becomes a matrix of the second order in the bases (2.1.1) and (2.1.2)

$$
\left\{\begin{array}{l}
\hat{T} \varphi=a \varphi+b \bar{\varphi}, \quad \hat{T} \bar{\varphi}=\bar{b} \varphi+\bar{a} \bar{\varphi} \\
\hat{T} c=\alpha_{11} c+\alpha_{12} s, \quad \hat{T} s=\alpha_{21} c+\alpha_{22} s .
\end{array}\right.
$$

From the invariance of the Wronskian it follows that det $\hat{T}=1$, or $|a|^{2}-|b|^{2}=1$ for real $k$, and $\alpha_{11} \alpha_{22}-\alpha_{21} \alpha_{12}=1$ for all $E$. The matrix $\hat{T}$ depends on $x_{0}$ and $E$ (or $k$ ). In the basis (2.1.2), $\hat{T}$ is an integral function of $E$. Under a change of the parameter $x_{0}$ the matrix $\hat{T}\left(x_{0}, k\right)$ changes to a similar matrix. Hence the dependence on $x_{0}$ is governed by the very useful equation

$$
\frac{d \widehat{T}}{d x_{0}}=[Q, \hat{T}],
$$

in which the matrix $Q$ is easily evaluated and in the bases (2.1.1) and (2.1.2) has the form

$$
\begin{aligned}
& \left.Q=i k\left(\begin{array}{rr}
1 & 0 \\
0 & -1
\end{array}\right)-\frac{i u}{2 k}\left(\begin{array}{ll}
1 & -1 \\
1 & -1
\end{array}\right) \quad \text { (basis }(2.1 .1)\right) \text {, } \\
& Q=\left(\begin{array}{cc}
0 & E-u \\
-1 & 0
\end{array}\right) \quad \text { (basis (2.1.2)). }
\end{aligned}
$$

The eigenfunctions ("Bloch" functions) are characterized by the requirements

$$
\hat{T} v_{ \pm}=e^{ \pm i p(E)} \psi_{ \pm},
$$

where $p(E)$ is called the quasi-momentum. For the Schrödinger operator it is convenient to normalize them by setting

$$
\psi_{ \pm}\left(x, x_{0}, E\right)=1 \text { for } x=x_{0} .
$$

If the potential $u(x)$ is real, then the solution zones (points of the spectrum) are determined, $p(E)$ being real and the functions $\psi_{ \pm}$almost periodic. The complements of the solution zones are called forbidden zones, 
lacunae, or zones of instability. As a rule, a typical potential has infinitely many lacunae, the lengths of which decrease rapidly as $E \rightarrow \infty$. The rate of decrease depends on the smoothness of the potential. If the potential is analytic, then the rate of decrease of the lengths of the lacunae is exponential.

The eigenvalues of the translation matrix $\hat{T}$ do not depend on the basis or on the choice of $x_{0}$; they determine the quasi-momentum $p(E)$ and the boundaries of the solution zones and forbidden zones. The trace of $\hat{T}$ has the form

$$
\begin{array}{ll}
\mathrm{Sp} \hat{T}=\alpha_{11}+\alpha_{22}=2 a_{\mathrm{Re}} & \text { (basis }(2.1 .1)), \\
\mathrm{Sp} \hat{T}=a+\bar{a}=2 a_{\mathrm{Re}} & \text { (basis (2.1.2)), }
\end{array}
$$

and the eigenvalues $\mu_{ \pm}=e^{ \pm i p(E)}$ are:

$$
\left\{\begin{aligned}
\mu_{ \pm}(E) & =a_{\mathrm{Re}} \pm i \sqrt{1-a_{\mathrm{Re}}^{2}} \\
a_{\mathrm{Re}} & =\cos p(E)=\frac{1}{2} \mathrm{Sp} \hat{T} .
\end{aligned}\right.
$$

It follows, clearly, from (2.1.8) that the periodic levels $\psi_{n}(x+T)=\psi_{n}(x), E=E_{n}$, are determined by

$$
\frac{1}{2} \mathrm{~S} p \hat{T}=a_{\mathrm{Re}}=1 .
$$

The antiperiodic levels $\psi_{m}(x+T)=-\psi(x)$ are determined by

$$
\frac{1}{2} \mathrm{Sp} \hat{T}=a_{\mathrm{Re}}=-1 \text {. }
$$

The periodic and antiperiodic levels can be simple (that is, singly degenerate) or doubly degenerate. In both cases we have $\mu_{ \pm}(E)= \pm 1$, but in the degenerate case $\hat{T}$ is diagonal:

$$
(2.1 .10) \hat{T}= \pm\left(\begin{array}{ll}
1 & 0 \\
0 & 1
\end{array}\right), \quad \text { or } b\left(E_{n}, x_{0}\right) \equiv 0, \quad \alpha_{21}\left(E_{n}, x_{0}\right) \equiv 0
$$

In the non-degenerate case $\hat{T}$ is a Jordan matrix and $\left|b\left(E_{n}, x_{0}\right)\right| \neq 0, \alpha_{21} \neq 0$. From the condition $|a|^{2}-|b|^{2}=1$ we have for $E=E_{n}$

$$
\left(2.1 .10^{\prime}\right) \quad\left|a_{\mathrm{Re}}\right|=1, \quad\left|a_{\mathrm{Im}}\right|=|b|, \quad E=E_{n},
$$

where $|b| \neq 0$. We already know that the boundaries of the forbidden and the solution zones are precisely the non-degenerate periodic and antiperiodic levels for which $|b| \neq 0, \alpha_{21} \neq 0$. Degenerate levels can be visualised as a forbidden zone contracted to a point. For example, if we increase the period to an integer multiple of itself, $T \rightarrow m T$, then we obtain

$$
\hat{T} \rightarrow \hat{T}^{m}, \quad e^{i p(E)} \rightarrow e^{i m p(E)} .
$$

The transformation (2.1.11) conserves the solution zones and forbidden zones, but inside the solution zones new degenerate levels appear when $m p(E)$ is a multiple of $2 \pi$.

As we know, $1-\frac{1}{2} \mathrm{Sp} \hat{T}$ is an entire function of order $\frac{1}{2}$ in the variable 
$E$, whose zeros, by (2.1.9), precisely determine all the periodic levels $E_{n}$. Therefore, the function $1-\frac{1}{2} \mathrm{Sp} \hat{T}$ can be represented as an infinite product, which is completely determined by the zeros. Hence, by $\left(2.1 .9^{\prime}\right)$ the antiperiodic levels are determined by the complete set of periodic levels (including the degenerate ones). However, we use as basic parameters only the boundaries of the zones, or the non-degenerate part of the periodic and antiperiodic levels (that is, the spectrum of the operator $L$ over the whole line).

The following simple proposition is extremely important for our situation.

LEMMA. For any (real or complex) smooth periodic potential $u(x)$, the Bloch eigenfunction $\psi_{ \pm}\left(x, x_{0}, E\right)$ defined by the conditions (2.1.6) and (2.1.7) is meromorphic on a two-sheeted Riemann surface $\Gamma$, covering the E-plane and having branch-points (for a real potential) at the ends of the zones. In general, this Riemann surface has infinite genus; however, if the number of lacunae is finite (this case is especially important for the theory of the $K-d V$ equation), then $\Gamma$ is hyperelliptic and has finite genus equal to the number of lacunae.

This proposition naturally leads to the following definition.

DEFINITION 1. A periodic potential $u(x)$ is said to be finite-zoned if the eigenfunction $\psi_{ \pm}\left(x, x_{0}, E\right)$ defined by the conditions (2.1.6) and (2.1.7) is meromorphic on a hyperelliptic Riemann surface $\Gamma$ of finite genus; the branch-points of the Riemann surface are said to be the "boundaries of the zones".

For later purposes we also need the following definition.

DEFINITION 1'. An almost periodic (real or complex) potential $u(x)$ is said to be finite-zoned if it has for all $E$ an eigenfunction $\psi(x, E)$, meromorphic on a hyperelliptic Riemann surface $\Gamma$ of finite genus, doubly covering the $E$-plane, with (2.1.6) modified as follows: the logarithmic derivative $\frac{d \ln (x)}{d x}$ is an almost-periodic function with the same group of periods as the potential $u(x)$.

It is also required that an $E \rightarrow \infty$ the function $\psi(x, E)$ has the asymptotic form $\psi \sim \exp \left\{ \pm i k\left(x-x_{0}\right)\right\}, k^{2}=E$. Then we denote $\psi$ by $\psi\left(x, x_{0}, E\right)$, where $\left.\psi\right|_{x=x_{0}}=1$, as in the periodic case. The logarithmic derivative $i \chi(x, E)=\frac{d \ln \psi}{d x}$ does not depend on $x_{0}$ and is a quantity of great importance in the theory of the operator $L=-\frac{d^{2}}{d x^{2}}+u$ and in the theory of the K-dV equation, as we have already indicated in Ch. 1 , §1. It satisfies the Riccati equation (1.1.4). If the potential is real, and in the solution zones for $E$ we set $\chi=\chi_{\mathrm{Re}}+i \chi_{\mathrm{Im}}$ then from the Riccati equation we deduce the relation

$$
\chi_{\operatorname{Im}}=\frac{1}{2}\left(\ln \chi_{\mathrm{Re}}\right)^{\prime}
$$


In the solution zones we have for the Wronskian $W$ of the Bloch functions

$$
W(\psi, \bar{\psi})=W\left(\psi_{+}, \psi_{-}\right)=2 i \chi_{\mathrm{Re}}\left(x_{0}, E\right) .
$$

By (2.1.13) the function equal to $\chi_{R}=\frac{1}{2 i} W\left(\psi_{+}, \psi_{-}\right)=\frac{1}{2 i}\left(\psi_{+}^{\prime} \psi_{-}-\psi_{+} \psi_{-}^{\prime}\right)$ is defined for all $E_{i}$ it is as the analogue to $\chi_{\mathrm{Re}}$ for complex potentials. We always denote this function by $\chi_{R}$. By definition, we have from (2.1.12)

(2.1.14) $\psi_{ \pm}\left(x, x_{0}, E\right)=\sqrt{\frac{\chi_{R}\left(x_{0}, E\right)}{\chi_{R}(x, E)}} \exp \left\{ \pm i \int_{x_{0}}^{x} \chi_{R}(x, E) d x\right\}$, from which it follows, by the definition of the quasi-momentum, that

$$
p(E)=\int_{x_{0}}^{x_{0}+T} \chi_{R}(x, E) d x
$$

Since $\cos p(E)=\frac{1}{2} \mathrm{Sp} \hat{T}$ by (2.1.8), and since the function $1-\frac{1}{2} \operatorname{Sp} \hat{T}$ is completely determined by the periodic spectrum, for example, as an infinite product, the integral $\int_{x_{0}}^{x_{0}+T} \chi_{R}(x, k) d x=\int_{x_{0}}^{x_{0}+T} \chi(x, k) d x$ and all the coefficients of its expansion in $\frac{1}{\sqrt{E}}=\frac{1}{k}$ as $E \rightarrow \infty$ (see (2.1.18) and below) are expressible in terms of the spectrum of the periodic problem (including the degenerate levels). These expressions are called "trace identities". In what follows it will become clear that all these quantities can be expressed in terms of only the boundaries of the zones.

It is convenient to express the function $\psi\left(x, x_{0}, E\right)$ in the basis (2.1.2):

(2.1.16) $\quad \psi\left(x, x_{0}, E\right)=c\left(x, x_{\mathrm{c}}, E\right)+i \alpha\left(x_{0}, E\right) s\left(x, x_{0}, E\right)$.

Evaluating the Wronskian $W(\psi, c)$, we obtain

$$
\alpha\left(x_{0}, E\right)=\chi\left(x_{0}, E\right) \text {. }
$$

For $p(E)$ we can obtain the important general relations when the potential varies

$$
\frac{d p(E)}{d E}=\int_{x_{0}}^{x_{0}+T} \frac{d x}{2 \chi_{R}(x, E)}, \quad \frac{\delta p(E)}{\delta u(x)}=-\frac{1}{2 \chi_{R}(x, E)},
$$

where $\delta p / \delta u$ is the variational derivative. A proof of these relations can be found in [40], [46].

As $|E| \rightarrow \infty, \chi(x, E)$ admits the asymptotic expansion

$$
\chi(x, E) \sim k+\sum_{n \geqslant 1} \frac{\chi_{n}(x)}{(2 k)^{n}}
$$

in which the expansion coefficients $\chi_{n}(x)$ can be found by recurrence formulae derived from the Riccati equation (1.1.4); they are polynomials 
in $u, u^{\prime}, u^{\prime \prime}, \ldots$ All the polynomials $\chi_{2 n}(x)$ are purely imaginary and by (2.1.12) they are total derivatives. Hence their integrals over a period vanish. A list of the first few polynomials $\chi_{2 n+1}(x)$ is given in Ch. $1, \S 1$ (see (1.1.3)).

Using the representation of $\psi_{ \pm}\left(x, x_{0}, E\right)$ in the form $(2.1 .16),\left(2.1 .16^{\prime}\right)$ it is not difficult to obtain an expression for $\chi(x, E)$ in terms of the coefficients of the translation matrix $\hat{T}$, since $\psi$ is an eigenvector of this matrix. In the bases (2.1.1) and (2.1.2) we obtain

$$
\left\{\begin{array}{l}
\chi_{R}(x, E)=\frac{k \sqrt{1-a_{\mathrm{Re}}^{2}}}{a_{\mathrm{Im}+b_{1 \mathrm{~m}}}} \quad(\text { basis }(2.1 .1)), k^{2}=E, \\
\chi_{R}(x, E)=\frac{\sqrt{1-\frac{1}{4}\left(\alpha_{11}+\alpha_{22}\right)^{2}}}{\alpha_{21}}
\end{array}\right. \text { (basis (2.1.2)), }
$$

where in the basis (2.1.1) the formula is valid only in a solution zone. The function $\psi_{ \pm}\left(x, x_{0}, E\right)$ can have poles at certain points $P_{1}, P_{2}, \ldots$ on $\Gamma$, depending, in general, on $x_{0}$ and $x$. For a real potential, these poles lie at the points of $\Gamma$ over points of the lacunae (or their boundaries) $\gamma_{1}\left(x_{0}\right), \ldots, \gamma_{m}\left(x_{0}\right), \ldots$, independent of $x$, one each for every lacuna (a pole occurs only on one sheet of $\Gamma$ over the point $\left.\gamma_{j}\left(x_{0}\right)\right)$. As is clear from (2.1.14), the zeros of $\psi_{ \pm}$lie over the points $\gamma_{j}(x)$, independent of $x_{0}$. If the potential is finite-zoned, having $n$ lacunae, then there are altogether $n$ zeros $P_{1}(x), \ldots, P_{n}(x)$ of $\psi_{ \pm}\left(x, x_{0}, E\right)$, lying over the points $\gamma_{1}(x), \ldots, \gamma_{n}(x)$ of the $E$-plane, and $n$ poles $P_{1}\left(x_{0}\right), \ldots, P_{n}\left(x_{0}\right)$ over the points $\gamma_{1}\left(x_{0}\right), \ldots, \gamma_{n}\left(x_{0}\right)$. For complex potentials the zeros and poles can be located arbitrarily on $\Gamma$.

We conclude this section by considering rapidly decreasing potentials for which the analytic properties of the eigenfunctions and the scattering matrices can be found in [5]. From our point of view, rapidly decreasing potentials are a degenerate limiting case of periodic potentials when $T \rightarrow \infty, u(x) \rightarrow 0$, as $|x| \rightarrow \infty$, and $u^{\prime}, u^{\prime \prime}, \ldots \rightarrow 0$ as $|x| \rightarrow \infty$ (more precisely: $\left.\int_{-\infty}^{\infty}(1+|x|)|u(x)| d x<\infty\right)$. Suppose to begin with that the potential $u(x)$ is of compact support (that is, $u$ is a finite function). We consider the bases of solutions analogous to (2.1.1) in which we set $x_{0}= \pm \infty$. We have two bases (the right and the left):

$$
\left\{\begin{aligned}
f_{+}(x, k) \rightarrow & e^{i k x}, \quad f_{-}(x, k) \rightarrow e^{-i k x}, \\
& (x \rightarrow+\infty), \\
g_{+}(x, k) \rightarrow & e^{i k x}, \quad g_{-}(x, k) \rightarrow e^{-i k x}, \\
& (x \rightarrow-\infty) .
\end{aligned}\right.
$$


Here the translation matrix for the period $T=\infty$ is the matrix of the transition from the basis $\left(g_{+}, g_{-}\right)$to the basis $\left(f_{+}, f_{-}\right)$. For real $k$ this matrix has the form

$$
\hat{T}=\cdot\left(\begin{array}{cc}
a & b \\
\bar{b} & \bar{a}
\end{array}\right), \quad|a|^{2}-|b|^{2}=1
$$

and $f_{+}=a g_{+}+b g_{-}, \hat{T}=\hat{T}(k)$.

The scattering matrix $\hat{S}$ is the matrix expressing the basis $\left(f_{-}, g_{+}\right)$in terms of the basis $\left(g_{-}, f_{+}\right)$; it is unitary and has the form

$$
\hat{S}=\left(\begin{array}{rr}
\frac{1}{a} & -\frac{b}{a} \\
\frac{b}{a} & \frac{1}{a}
\end{array}\right) .
$$

The coefficients $s_{11}=1 / a$ and $-s_{12}=b / a$ are called transmission and reflection coefficients (the scattering amplitudes in the directions 0 and $\pi$ ).

We note that for finite potentials $\hat{T}(k)$ can be continued analytically as an entire function of $k$; if $\operatorname{Im} k>0$, then $f_{+}$decreases and $g_{-}$decreases, as $x \rightarrow+\infty$. From the equation $f_{+}=a(k) g_{+}+b(k) g_{-}$it follows that we obtain a solution that decreases in both directions $(|x| \rightarrow \infty)$ when $a\left(k_{n}\right)=0$ (or the amplitude $s_{11}(k)=\frac{1}{a}$ has a pole). There are finitely many discrete levels $E_{n}=k_{n}^{2}$; the functions $f_{+}(x, k)$ and $a(k)$ are analytic for $\operatorname{Im} k>0$, and

$$
\text { (2.1.23) } \quad a(k) \rightarrow 1+O(1 / k), \quad|k| \rightarrow \infty, \quad \operatorname{Im} k>0 .
$$

The eigenfunctions of the discrete spectrum for the levels $E_{n}=k_{n}^{2}$, where $a\left(k_{n}\right)=0$, have the form

$$
\psi_{n}(x)=g_{-}\left(x, k_{n}\right)
$$

the normalization factors are easily evaluated:

$$
\int_{-\infty}^{\infty}\left|\psi_{n}\right|^{2} d x=\frac{1}{c_{n}}=\frac{\left.i \frac{d a}{d k}\right|_{k=i \varkappa_{n}}}{b\left(i x_{n}\right)} .
$$

In the general case of a rapidly decreasing potential, according to [5] we have functions $a(k), b(k)$, where $a(k)$ is analytic in the upper half-plane Im $k>0$, has the asymptotic form (2.1.23) and the relation $|a|^{2}-|b|^{2}=1$ holds on the real axis $\operatorname{Im} k=0$. In addition, there are finitely many zeros $k_{n}, a\left(k_{n}\right)=0$ (all the $k_{n}$ are purely imaginary) and corresponding numbers $c_{n}$ given by (2.1.25). From the uniqueness theorem of Marchenko [4] it follows that $\left(a(k), b(k), k_{n}, c_{n}\right)$ is a set of "scattering data", completely determining the potential $u(x)$. Moreover, the kernel 


$$
F(z+t)=\int_{-\infty}^{\infty} e^{-i k(z+t)} \frac{b(k)}{a(x)} d k+\sum_{n} c_{n} e^{-i k_{n}(z+t)}
$$

is well-defined, and we can write down the Gel'fand-Levitan equation

$$
K(k, z)+F(x+z)+\int_{-\infty}^{x} F(s+x) K(z, s) d s=0 .
$$

The potential itself is obtained from the formula $u(x)=2 \frac{d}{d x} K(x, x)$.

When $a(k)$ has the indicated analytic properties [5], the Gel'fand-Levitan equation (2.1.27) has one and only one solution. An important special case is that for which $b \equiv 0$ on the real $k$-axis. Such potentials are called "nonreflecting". In this case the equation (2.1.27) becomes algebraic, and the potential $u(x)$ is a rational function of the exponentials $e^{x_{1} x}, e^{\chi_{2} x}, \ldots, e^{x_{n} x}$, in which $k_{n}=i \chi_{n}=\sqrt{ } E_{n}$. The function $a(k)$ then has the form

$$
a(k)=\prod_{n} \frac{k-i x_{n}}{k+i x_{n}}
$$

These potentials were first found by Borg [1] and Bargmann [2]. They play an important part in the theory of the $\mathrm{K}-\mathrm{dV}$ equation. They are also important to us in our concern with periodic problems, because as $T \rightarrow \infty$, the finite-zone potentials degenerate into non-reflecting ones; the solution zones shrink to isolated points of the discrete spectrum inside the solution zones $b \equiv 0$ for $T=\infty$, by (2.1.10) and (2.1.11), and $\Gamma$ degenerates into a rational surface, since the pairs of branch-points coalesce.

\section{§2. A new commutation representation of the K-dV and "higher order K-dV" equations. An algorithm for finding finite-zone potentials and their spectra}

In Ch. 1, $\S 1$ we have recalled the Lax representation (1.1.1) and (1.1.1') of the $\mathrm{K}-\mathrm{dV}$ equation and its higher order analogues in the form $\frac{d L}{d t}=\left[A_{n}, L\right]$, where $\dot{u}=Q_{n}\left(u, u^{\prime}, u^{\prime \prime}, \ldots, u^{(2 n+1)}\right)$. We now reproduce the algorithm [18] for integrating the Cauchy problem for K-dV equations with rapidly decreasing functions. This algorithm is based on the following equation for the translation (or monodromy) matrix $\hat{T}(k)$, discovered in [18] :

$$
\dot{a}(k)=0, \quad \dot{b}(k)=-8 i k^{3} b(k) .
$$

For finite potentials we obtain from (2.2.1) the following equation for the discrete levels, using (2.1.24) and (2.1.25): 


$$
\dot{\varkappa}_{n}=\left(\sqrt{-E_{n}}\right)^{\circ}=0, \quad \dot{c}_{n}=-8 x_{n}^{3} c_{n},
$$

since $k_{n}=i x_{n}$ are the zeros of the function $a(k)$, and $c_{n}=\left(\frac{d a}{d k}\right)_{k=k_{n}} \frac{1}{b\left(k_{n}\right)}$. Therefore, (2.2.1) and (2.2.1') hold for any rapidly decreasing potential and completely determine the time-development of the scattering data (and hence of the potential itself). Let us derive the equations (2.2.1). We consider the eigenfunction $f_{+}(x, k)$ defined by (2.1.20). Clearly, $\frac{\partial}{\partial t} f_{+}(x, k) \rightarrow 0$, as $x \rightarrow+\infty$. Since $L f_{+}=k^{2} f_{+}$, for the derivative $\left(\left(L-k^{2}\right) f_{+}\right)^{\bullet}$ we have

$$
\dot{L} f_{+}+\left(L-k^{2}\right) \dot{f}_{+}=(A L-L A) f_{+}+\left(L-k^{2}\right) \dot{f}_{+}=\left(L-k^{2}\right)\left(\dot{f}_{+}-A f_{+}\right) .
$$

From this we obtain

$$
\dot{f}_{+}=A f_{+}+\lambda(k) f_{+}+\mu(k) f_{-} .
$$

By letting $x \rightarrow \infty$, we obtain

$$
0 \sim\left(A f_{+}\right)_{x \rightarrow+\infty}+\left(\lambda f_{+}\right)_{x \rightarrow+\infty}+\left(\mu f_{-}\right)_{x \rightarrow+\infty}
$$

consequently, since $A$ has the form $A=-4 \frac{d^{3}}{d x^{3}}+3\left(u \frac{d}{d x}+\frac{d}{d x} u\right)$ and $u, u^{\prime} \rightarrow 0$, we finally have

$$
\left(A f_{+}\right)_{x \rightarrow+\infty} \rightarrow 4 i k^{3} e^{i k x}, \quad \mu(k) \equiv 0, \quad \lambda(k)=-4 i k^{3} .
$$

We now recall the equation $f_{+}=a g_{+}+b g_{-}(2.1 .21)$ and let $x_{0} \rightarrow-\infty$. Then $g_{+} \rightarrow e^{i k x}, g_{-} \rightarrow e^{-i k x}$. From this it follows that $\dot{f}_{+} \rightarrow a e^{i k x}+b e^{-i k x}$, as $x \rightarrow-\infty$. Comparing these formulae with (2.2.2) and bearing (2.2.3) in mind, we finally obtain (2.2.1): $a=0, b=-8 i k^{3} b$. Now (2.2.1') follows from this, as indicated above, and the integration of the $\mathrm{K}-\mathrm{dV}$ equation for rapidly decreasing functions is complete.

For the higher order K-dV equations we have

$$
A_{n}=\frac{d^{2 n+1}}{d x^{2 n+1}}+\sum_{i=0}^{2 n} P_{i}\left(u, u^{\prime}, \ldots\right) \frac{d^{i}}{d x^{i}}, \quad \text { where } P_{i} \equiv 0 \text { when } u \equiv 0 .
$$

By analogy with the preceding derivation it follows for $\hat{T}(k)$ that

$$
\dot{a}=0, \quad \dot{b}=\mathrm{const}(i k)^{2 n+1} \text {. }
$$

We see from (2.2.4) that all the higher order K-dV equations commute as dynamical systems in the function space of rapidly decreasing functions.

Gardner [22] has shown that all the higher order K-dV equations have the Hamiltonian form

$$
\dot{u}=\frac{\partial}{\partial x} \frac{\delta I_{n}}{\delta u(x)},
$$

as already stated in Ch. $1, \S 1$, where the $I_{n}$ are the Hamiltonians. From the commutativity of these dynamical systems it follows that the Poisson 
brackets of the functionals $I_{n}$ vanish:

$$
\left[I_{n}, I_{m}\right]=0 .
$$

since the skew-symmetric form is determined by $\frac{\partial}{\partial x},(2.2 .6)$ is equivalent to

$$
\int_{-\infty}^{\infty} \frac{\delta I_{n}}{\delta u(x)} \frac{\partial}{\partial x} \frac{\delta I_{m}}{\delta u(x)} d x=0
$$

for any rapidly decreasing function $u(x)$. Since $I_{n}$ and $I_{m}$ are integrals of expressions that are polynomials in $u, u^{\prime}, u^{\prime \prime}$, with constant coefficients, it now becomes absolutely clear that the identity (2.2.6) also holds for any periodic function $u(x)$. Gardner proved in [22] the commutativity relation (2.2.6) by direct calculations. Simultaneously, Faddeev and Zakharov in [21] computed all the Poisson brackets of all the "scattering data" $a(k), b(k), x_{n} c_{n}$ and proved that

$$
\begin{cases}P_{k}=\frac{2 k}{\pi} \ln |a(k)|, & P_{n}=x_{n}^{2}, \\ Q_{k}=\arg b(k), & Q_{n}=\ln b_{n}, \\ \text { where } b_{n}=\left.i c_{n}(d a / d k)\right|_{k=i x_{n},}\end{cases}
$$

are canonical variables ("action-angle" variables) for all the Hamiltonian systems (2.2.5) and all the $\mathrm{K}-\mathrm{dV}$ equations.

Great difficulties arise in attempts to generalize this method of integrating the K-dV equation to the periodic case. These will become clearer after we have derived the natural analogue to $(2.2 .1)$ for $\hat{T}$. We consider the bases (2.1.1) or (2.1.2) and compute the time derivatives by analogy with (2.2.2) at $x=x_{0}$ instead of $x=\infty$ :

$$
\left\{\begin{array}{l}
\dot{\varphi}_{+}=A \varphi_{+}+\lambda_{11} \varphi_{+}+\lambda_{12} \varphi_{-} \\
\varphi_{-}=A \varphi_{-}+\lambda_{21} \varphi_{+}+\lambda_{22} \varphi_{-}
\end{array}\right.
$$

in the basis (2.1.1), or

$$
\left\{\begin{array}{l}
\dot{c}=A c+a_{11} c+a_{12} s \\
\dot{s}=A s+a_{21} c+a_{22} s
\end{array}\right.
$$

in the basis (2.1.2), where $\lambda_{11}=\lambda, \lambda_{12}=\mu, \lambda_{21}=\bar{\mu}, \lambda_{22}=\bar{\lambda}$ for real $k$ and all the $a_{i j}$ are real for real $E$. We have the matrix $\Lambda=\left(\begin{array}{ll}\lambda_{11} & \lambda_{12} \\ \lambda_{21} & \lambda_{22}\end{array}\right)$, or $\Lambda=\left(\begin{array}{ll}a_{11} & a_{12} \\ a_{21} & a_{22}\end{array}\right)$, in the bases (2.1.1) and (2.1.2), respectively, the coefficients of which can be expressed in terms of $u\left(x_{0}\right), u^{\prime}\left(x_{0}\right), \ldots$ and $k$ by the following formulae:

$$
\left\{\begin{array}{c}
\left.\dot{\varphi}_{+}\right)_{x=x_{0}}=0=\left(A \varphi_{+}\right)_{x=x_{0}+\lambda_{11}+\lambda_{12}} \\
\left(\varphi_{+}=1 \text { for } x=x_{0}\right), \\
\left(\frac{d}{d x} \dot{\varphi}_{+}\right)_{x=x_{0}}=0=\left(\frac{d}{d x} A \varphi_{+}\right)_{x=x_{0}}+i k\left(\lambda_{11}-\lambda_{12}\right) .
\end{array}\right.
$$


For real $k$ we have

$$
\Lambda=\left(\frac{\lambda}{\mu} \frac{\mu}{\lambda}\right), \quad \lambda=\lambda_{11}, \quad \mu=\lambda_{12},
$$

where the trace $\operatorname{Sp} \Lambda=0$ or $\lambda+\bar{\lambda}=0$, and the coefficients $\lambda, \mu$ are as in (2.2.9). The matrix $\Lambda=\left(a_{i j}\right)$ for the basis (2.1.2) is obtained in exactly the same way. We note that in the basis (2.1.2) the coefficients $a_{i j}$ of $\Lambda$ are polynomials in $k^{2}=E, u\left(x_{0}\right), u^{\prime}\left(x_{0}\right), \ldots$ The determinant det $\Lambda$ does not depend on the choice of basis and is a polynomial

$$
\operatorname{det} \Lambda=P_{2 n+1}(E) \text {, }
$$

the operator $A=A_{n}$ being of degree $2 n+1$. To calculate the dynamics of the matrix $\hat{T}\left(x_{0}, E\right)$ in any of the bases we must compute (2.2.8) for $x=x_{0}+T$, where $T$ is the period. For example, in the basis (2.1.1) and for real $k$ we obtain

$$
\left\{\begin{array}{c}
{\left[\dot{\varphi}_{+}\right]_{x=x_{0}+T}=\dot{a}+\dot{b}=\left[A\left(a \varphi_{+}+b \varphi_{-}\right)\right]_{x=x_{0}+T}+} \\
+\lambda(a+b)+\mu(\bar{b}+\bar{a}), \\
{\left[\frac{d}{d x} \dot{\varphi}_{+}\right]_{x=x_{0}+T}=i k(\dot{a}-\dot{b})=\left[\frac{d}{d x} A\left(a \varphi_{+}+b \varphi_{-}\right)\right]_{x=x_{0}+T}+} \\
+\lambda i k(a-b)+\mu i k(\bar{b}-\bar{a}) .
\end{array}\right.
$$

Bearing in mind that $A$ is real, we finally obtain without difficulty

$$
\dot{a}=\mu \bar{b}-b \bar{\mu}, \quad \dot{b}=(\lambda-\bar{\lambda}) b+(a-\bar{a}) \mu,
$$

where

$$
\Lambda=\left(\frac{\lambda}{\mu} \frac{\mu}{\lambda}\right) .
$$

Clearly, the equations (2.2.13) are equivalent to the matrix equation

$$
\frac{\partial}{\partial t} \hat{T}=[\Lambda, \hat{T}] \text {, }
$$

where the matrix $\Lambda$ is defined by (2.2.8) and in the basis (2.1.2) depends polynomially on $E, u\left(x_{0}\right), u^{\prime}\left(x_{0}\right), \ldots, u^{(2 n)}\left(x_{0}\right)$. In the form (2.2.14) the equation holds, of course, in any basis. If at a point $x_{0}$ we have $u=u^{\prime}=\ldots=0$, then in the basis (2.1.1) we easily obtain

$$
\Lambda=\mathrm{const}\left(\begin{array}{cc}
i k^{2 n+1} & 0 \\
0 & -i k^{2 n+1}
\end{array}\right) \text {. }
$$

Substituting (2.2.15) in (2.2.14) we clearly obtain the Gardner-GreenKruskal-Miura formulae for rapidly decreasing potentials by setting $x_{0}= \pm \infty$. In the periodic case, (3.2.14) can no longer be integrated. For the initial K-dV equation (2.2.14) the formula in the basis (2.1.2) was derived by another method by Marchenko [44], who went further in constructing a method of successive approximations for solving this equation.

We shall proceed in a different way. The matrix $\hat{T}$ depends on the 
parameters $x_{0}, t$, and $E$. We consider for $\hat{T}$ the pair of equations (2.2.14) and (2.1.5):

$$
\frac{\partial}{\partial t} \hat{T}=[\Lambda, \hat{T}], \quad \frac{\partial}{\partial x_{0}} \hat{T}=[Q, \hat{T}] .
$$

From the condition for compatibility of this pair of equations, $\frac{\partial}{\partial x_{0}} \quad \frac{\partial}{\partial t} \hat{T}=\frac{\partial}{\partial t} \frac{\partial}{\partial x_{0}} \hat{T}$, we obtain

$$
\left[\frac{\partial \Lambda}{\partial x_{0}}-\frac{\partial Q}{\partial t}-[\Lambda, Q], \hat{T}\right]=0
$$

from which it follows, since the trace of the left-hand side vanishes, that

$$
\frac{\partial \Lambda}{\partial x_{0}}-\frac{\partial Q}{\partial t}=[\Lambda, Q] \text {. }
$$

Now (2.2.17) gives a new and very convenient commutation representation of the K-dV and all "higher order K-dV" equations by second order matrices that depend polynomially on $E$ (in the base (2.1.2)). An analogue of this commutation representation as well as analogues of (2.2.14) and (2.1.5) can also be obtained naturally for all other non-linear systems mentioned in Ch. 1 (see Ch. 3).

We now consider the "general K-dV equation"

$$
\dot{u}=\frac{\partial}{\partial x}\left(\sum_{i=0}^{n} c_{i} \frac{\delta I_{n-i}}{\delta u(x)}\right) \quad\left(c_{0}=1\right)
$$

and the equivalent Lax equation

$$
\dot{L}=[A, L], \text { where } A=\sum_{i=0}^{n} c_{i} A_{n-i} .
$$

The matrix $\Lambda$ constructed according to (2.2.8) depends additively on $A$, and we have the new representation (2.2.17)

$$
\frac{\partial \Lambda}{\partial x_{0}}-\frac{\partial Q}{\partial t}=[\Lambda, Q], \quad \Lambda=\sum_{i=0}^{n} c_{i} \Lambda_{n-i} .
$$

Suppose that we wish to find a stationary solution $\dot{u}=0$ of the higher order K-dV equation (2.2.18). If $\dot{u}=0$, then $\frac{\partial Q}{\partial t}=0$ and we obtain

$$
\frac{d \Lambda}{d x_{0}}=[\Lambda, Q] \text {. }
$$

Thus, we have proved the following result.

COROLLARY 1 (see [38]). The system of ordinary equations

$$
\sum c_{i} \frac{\delta I_{n-i}}{\delta u(x)}=\mathrm{const}
$$

is equivalent to the equation of Lax type (2.2.21) with second order matrices that are polynomially dependent on the extra parameter $E$ and are polynomial in $u(x), u^{\prime}(x), \ldots$ In particular, all the coefficients of the 
characteristic polynomial $P(E)$, where

$$
W^{2}+P(E)=\operatorname{det}(W-\Lambda)=W^{2}+\operatorname{det} \Lambda(\operatorname{Sp} \Lambda=0),
$$

are polynomials in $u, u^{\prime}, u^{\prime \prime}, \ldots$ independent of $x$ (that is, they are integrals of the system (2.2.22)). The polynomial $P(E)$ itself is of degree $2 n+1$.

We now prove the following beautiful and unexpected corollary of equation (2.2.14).

COROLLARY 2. The roots of the polynomial $P(E)=0$ are the complete set of boundaries of the forbidden and of the solution zones of the

Schrödinger operator $L$ with potential $u(x)$ satisfying the equation (2.2.22) for the stationary solutions of any higher order $K-d V$ equation. In particular, all the stationary solutions of the $K-d V$ equations are potentials whose number of zones does not exceed $n$.

DERIVATION OF COROLLARY 2. From (2.2.14) $\dot{\hat{T}}=[\Lambda, \hat{T}]$ we obtain $\dot{\hat{T}}=0$ or $[\Lambda, \hat{T}]=0$. We consider the matrix element (in the base (2.1.1) $[\Lambda, \hat{T}]_{12}=(a-\bar{a}) \mu+(\lambda-\bar{\lambda}) b$ for real $k$. Since $\bar{\lambda}=-\lambda$, we have $2 \mu a_{\mathrm{lm}}=2 \lambda b$. From this it follows that in all the non-degenerate levels (boundaries of the zones) for which $|b| \neq 0$

$$
\left|\frac{a_{\operatorname{Im}}}{b}\right|=\left|\frac{\lambda}{\mu}\right|,
$$

or det $\Lambda=P(E)=|\lambda|^{2}-|\mu|^{2}$ when $E=E_{n}$. By adding a constant to $u$ we can always achieve that all $E_{n}>0$ and the $k_{n}=\sqrt{ } E_{n}$ are real. Thus, Corollary 2 is proved. This proof is taken from [38]. Another derivation of Corollary 2 was indicated by Dubrovin in connection with a generalization of these results to matrix systems (see Ch. 3): since by the definition (2.1.6) the Bloch function $\psi_{+}\left(x, x_{0}, E\right)$ is an eigenfunction for $\hat{T}, \hat{T} \psi_{ \pm}=e^{i p(E)} \psi_{ \pm}$, it follows from $[\Lambda, \hat{T}]=0$ that it is also an eigenvector for the matrix $\Lambda$. Since $\Lambda$ depends polynomially on $E$, the eigenvector $\psi_{ \pm}$ is meromorphic on the Riemann surface $\Gamma$ :

$$
R(W, E)=\operatorname{det}(W-\Lambda)=0 .
$$

In our case $\operatorname{Sp} \Lambda=0$, and we have

$$
R(W, E)=W^{2}+P_{2 n+1}(E)=0,
$$

where $P_{2 n+1}(E)=\operatorname{det} \Lambda$. The branch-points of $\Gamma$ are the boundaries of the zones, as indicated in $\mathrm{Ch} .2, \S 1$. It is important that this derivation easily generalizes not only to complex potentials, but also to almost-periodic potentials and to other linear operators.

A third derivation, which yields part of the result of Corollary 2, was obtained by Lax [50] simultaneously with, and independently of, [38], as was mentioned in the Introduction. In fact, following [50] we prove that every periodic stationary solution of any "higher order K-dV equation" has only finitely many forbidden zones in the spectrum of the operator 
$L=-\frac{d^{2}}{d x^{2}}+u(x)$. For the sake of simplicity, we produce Lax's derivation for the original $\mathrm{K}-\mathrm{dV}$ equation. Let $f_{n}$ be a non-degenerate periodic eigenfunction. We consider a normalization of the eigenfunction $f_{n}(x)$ instead of the bases (2.1.1) and (2.1.2) in which $L f_{n}=E$, such that the development in time has the form

$$
\dot{f}_{n}=A f_{n} .
$$

Since $L f_{n}=E_{n} f_{n},(2.2 .25)$ leads to a first order equation in $x$, which for $A=-4 \frac{d^{3}}{d x^{3}}+3\left(u \frac{d}{d x}+\frac{d}{d x} u\right)$ is as follows:

$$
\dot{f}_{n}=(4 E+2 u) f_{n_{x}}-u_{x} f_{n_{x}} .
$$

The characteristics of the equation have the form

$$
\frac{d x}{d t}=-(4 E+2 u)
$$

and along the characteristics we have

$$
\frac{d f_{n}}{d t}=u_{x} f_{n} .
$$

Hence the zeros of $f_{n}$ are situated along the characteristics. For stationary solutions of the form $u(x-c t)$ (that is, $\left.A=A_{1}+c A_{0}\right)$ we have

$$
f_{n}=f_{n}(x-c t), \quad \frac{d x}{d t}=c, \quad x=x_{0}+c t .
$$

Comparing (2.2.28) and (2.2.26), we obtain for the zeros of $f_{n}$ :

(2.2.29) $\frac{d x}{d t}=c=-\left(4 E_{n}+2 u(x-c t)\right)=-4 E_{n}-2 u\left(x_{0}\right)$.

For large values of $n$ the number $E_{n}$ is large and (2.2.29) cannot hold. This means that either the eigenfunction $f_{n}$ has no zeros, or that it cannot be non-degenerate. We know that eigenfunctions corresponding to high levels $E_{n} \rightarrow 0$ have more and more zeros. In consequence, all but finitely many levels are degenerate. A similar derivation for antiperiodic levels (period $2 T$ ) shows that there are finitely many zones. It is easy to see that this derivation generalizes also to higher order $\mathrm{K}-\mathrm{dV}$ equations. From this derivation it does not follow that the number of lacunae is $\leqslant n$ for the $n$th analogue of the $\mathrm{K}-\mathrm{dV}$ equation (for the excluded case $n=1$ see [50]). Moreover, this derivation, unlike Corollaries 1 and 2 above, does not give an algorithm for the integration of the stationary problem for higher order K-dV equations nor an algorithm for finding the boundaries of the zones.

We now pass on to the most recent work on stationary solutions of higher order K-dV equations. All the (2.2.22) are equations for extremals of the functional

$$
\delta\left(d I_{-1}+\sum_{i=0}^{n} c_{i} I_{n-1}\right)=0 \quad\left(c_{0}=1\right),
$$


where $I_{-1}=-\int u d x$. Therefore, all the equations (2.2.30) are Hamiltonian systems with $n$ degrees of freedom, depending on the $(n+1)$ constants $d, c_{1}, \ldots, c_{n}$. The coefficients of the polynomial $P_{2 n+1}(E)=\operatorname{det} \Lambda$ are constructed in such a way that the leading one is a constant, then the next $n+1$ are formed from the parameters $\left(c_{1}, c_{2}, \ldots, c_{n}, d\right)$ and the last $n$, denoted by $J_{1}, \ldots, J_{n}$, give a set of polynomials that are algebraically independent integrals of the system (2.2.22). In fact, the integrals $J_{1}, \ldots, J_{n}$ are involutory and hence the system (2.2.22) is totally integrable, and its solutions, in principle, can be determined by the Liouville algorithm. (It will become clear later that the Hamiltonians $J_{\alpha}$ give a set of independent commuting systems, hence we do not prove this here.) Following the method applied above in the derivation of (2.2.17) from (2.2.14) and (2.1.5), we use the commutativity of all the higher order K-dV equations as dynamical systems in a function space. If we have two equations

$$
\begin{aligned}
\frac{\partial u}{\partial t_{m}} & =\frac{\partial}{\partial x} \frac{\delta I_{m}}{\delta u(x)}, \\
\frac{\partial u}{\partial t} & =\frac{\partial}{\partial x} \sum_{i=0}^{n} c_{i} \frac{\delta I_{n-i}}{\delta u(x)},
\end{aligned}
$$

then the solution is a function $u\left(x, t, t_{m}\right)$ by virtue of the commutativity of these systems. For the matrix $\hat{T}\left(x_{0}, E\right)$ we obtain

$$
\text { (2.2.31) } \quad \frac{\partial \hat{T}}{\partial t_{m}}=\left[\Lambda_{m}, \hat{T}\right], \quad \frac{\partial \hat{T}}{\partial t}=[\Lambda, \hat{T}]
$$

where $\Lambda=\sum_{i=0}^{n} c_{i} \Lambda_{n-i}$. If $u(x)$ is a stationary solution, that is, $\frac{\partial u}{\partial t}=0$ (so that $u$ is a solution of $(2.2 .22)$ ), then

$$
\frac{\partial \hat{T}}{\partial t}=\frac{\partial \Lambda_{m}}{\partial t}=0 \text {. }
$$

In all cases we obtain from the compatibility of the two equations (2.2.31), by analogy with (2.2.17),

$$
\frac{\partial \Lambda_{m}}{\partial t}-\frac{\partial \Lambda}{\partial t_{m}}=\left[\Lambda, \Lambda_{m}\right]
$$

If $\frac{\partial \hat{T}}{\partial t}=\frac{\partial \Lambda}{\partial t} m=0$, then

$$
\frac{d \Lambda}{d t_{m}}=\left[\Lambda_{m}, \Lambda\right]
$$

When $m=0$, (2.2.33) becomes (2.2.21) with $\Lambda_{0}=Q$. Clearly, (2.2.33) is defined over the same phase space as (2.2.21) and gives a set of commuting Hamiltonians of dynamical systems on the phase space of the problem (2.2.21) (or on the set of stationary solutions of the higher order K-dV equations, given by (2.2.22)).

When $m=1,(2.2 .33)$ determines the dynamics of finite-zone potentials under time evolution governed by the $\mathrm{K}-\mathrm{dV}$ equation, which is described 
explicitly in the form of a finite-dimensional dynamical system, represented by a matrix equation for second order matrices polynomially dependent on the parameter $E$ (in the basis (2.1.2)). Here, the matrix $\Lambda$ replaces the Schrödinger operator $L$ and the characteristic polynomial of $\Lambda$ has the form $R(W, E)=\operatorname{det}(W-\Lambda)$, and the Riemann surface $R(W, E)=0$ is also the "spectrum" (in the sense of Ch. 2, §1) of the potential we are studying.

We exhibit the matrix $\Lambda_{0}=Q, \Lambda_{1}, \Lambda_{2}$ in the basis (2.1.1) for real $k$, the corresponding polynomials $P_{3}(E), P_{5}(E)$, and the integrals $J$ (for $n=1$ ), $J_{1}$ and $J_{2}$ for $n=2$. The matrix $\Lambda=\left(\frac{\lambda}{\mu} \frac{\mu}{\lambda}\right)$ has the following form:

$$
\text { 1) } n=0 ; \quad \lambda=i k-\frac{i u}{2 k}, \quad \mu=\frac{i u}{2 k}, \quad R_{1}=E-c,
$$

and the potential has the form $u=c$.

$$
\begin{gathered}
\text { 2) } n=1 ; \quad \Lambda=\Lambda_{1}+c \Lambda_{0}, \\
\lambda=\frac{i}{k}\left(-\frac{u^{r}}{2}+u^{2}-4 k^{4}+c k^{2}-c \frac{u}{2}\right), \\
\mu=u^{\prime}+\frac{i}{k}\left(\frac{u^{n}}{2}-u^{2}-2 k^{2} u+c \frac{u}{2}\right), \\
R_{3}(E)=|\lambda|^{2}-|\mu|^{2}=E^{3}+\frac{1}{2} c E^{2}+\frac{1}{16}\left(c^{2}-4 d\right)+\frac{1}{16}(c d-J), \\
J=\left(u^{\prime}\right)^{2}-\left(2 u^{3}+c u^{2}+2 d u\right) ;
\end{gathered}
$$

the potential has the form

$$
x-x_{0}=\int \frac{d u}{\sqrt{2 u^{3}+c u^{2}+2 d u+J}} .
$$

3) $n=2 ; \Lambda=\Lambda_{2}+c_{2} \Lambda ; \quad\left(c_{1}=0\right)$,

$$
\lambda=\frac{i}{k}\left[\frac{1}{2} u^{\mathrm{IV}}-\left(4 u u^{\prime \prime}+3\left(u^{\prime}\right)^{2}-3 u^{3}\right)-2 u^{2} k^{2}+\right.
$$

$$
\left.+16 k^{6}+c_{2} k^{2}-c_{2} \frac{u}{2}\right] \text {, }
$$

$$
\mu=-u^{\prime \prime \prime}+6 u u^{\prime}-4 u^{\prime} k^{2}+\frac{i}{k}\left[\frac{1}{2} u^{\mathrm{IV}}+4 u u^{\prime \prime}+3\left(u^{\prime}\right)^{2}-\right.
$$

$$
\begin{gathered}
\left.-3 u^{3}+k^{2}\left(2 u u^{\prime \prime}-4 u^{2}\right)-8 u k^{4}+c_{2} \frac{n}{2}\right], \\
\left.P_{5}(E)=|\lambda|^{2}-|\mu|^{2}=E^{5}+\frac{1}{4} c_{2} E^{3}-\frac{1}{16} d E^{2}+\frac{1}{32} J_{1}+\frac{1}{4} c_{2}^{2}\right) E+\frac{J_{2}}{2^{8}}+\frac{c_{2} d}{2^{7}}, \\
J_{1}=p_{1} p_{2}-\left(\frac{1}{2} q_{2}^{2}+\frac{5}{2} q_{1}^{2} q_{2}+\frac{5}{8} q_{1}^{4}\right)+c_{2} q_{1}^{2}-d q_{1}, \\
J_{2}=p_{1}^{2}-2 q_{1} p_{1} p_{2}+2\left(q_{2}-c_{2}\right) p_{2}^{2}+q_{1}^{5}+2 c_{2} q_{1}^{3}+d q_{1}^{2}-4 q_{1} q_{2}^{2}+4 c_{2} q_{1} q_{2}-2 d q_{2} .
\end{gathered}
$$

The Hamiltonian system (2.2.22) for $c_{1}=0$ is given by the Hamiltonian $H=J_{1}$, and the canonical coordinates are:

$$
p_{1}=q_{2}^{\prime}, \quad p_{2}=u^{\prime}, \quad q_{1}=u, \quad q_{2}=-\frac{5}{2} u^{2}+u^{\prime \prime} .
$$


In particular, for the Lamé potential $3 u(x)$, where $u(x)=2 \wp(x), \wp(x)$ being the Weierstrass elliptic function, we can exhibit the boundaries of the zones: if $\left(8^{\prime}\right)^{2}=48^{3}-g_{2} 8^{\circ}-g_{3}$ and $e_{1}, e_{2}, e_{3}$ are the roots of the polynomial $4 x^{3}-g_{2} x-g_{3}$ (all $e_{i}$ are real, $e_{1}<e_{2}<e_{3}$ ), then the boundaries of the zones are

(2.2.38) $\quad E_{1}=3 e_{1}, \quad E_{2}=-\sqrt{3 g_{2}}, \quad E_{3}=3 e_{2}, \quad E_{4}=\sqrt{3 g_{2}}, \quad E_{5}=3 e_{3}$.

Convenient coordinates $\gamma_{1}, \gamma_{2}$ on the level surfaces $J_{1}=$ const, and $J_{2}=$ const are given as follows (let $\sum_{i=1}^{5} E_{i}=0$ ):

$$
\left\{\begin{array}{l}
2\left(\gamma_{1}+\gamma_{2}\right)=u=q_{1}, \\
\gamma_{1,2}=-\frac{1}{4}\left[q_{1} \pm \sqrt{2 q_{2}-8 \sum E_{i} E_{j}}\right],
\end{array}\right.
$$

and all $p_{1}, p_{2}, q_{1}, q_{2}$ can be expressed in terms of $\gamma_{1}$ and $\gamma_{2}$. The interpretation of the coordinates $\gamma_{1}$ and $\gamma_{2}$, the formation of "angle variables" from them, and the completion of the integration of the equation (2.2.22) for $n=2$ will be discussed in $\$ 3$ (see (2.3.14)). In general, the real solutions of (2.2.22) are almost-periodic functions with a group of periods $T_{1}, \ldots, T_{n}$. Bounded solutions correspond to tori in the phase space, and the group of periods can be expressed in terms of the boundaries of the zones, or, what amounts to the same thing, in terms of the constants $\left(c_{1}, \ldots, c_{n}, d\right)$ and the integrals $J_{1}, \ldots, J_{n}$. Later, in $\S 3$, we shall give convenient formulae for the periods $T_{1}, \ldots, T_{n}$ and for the potentials themselves. The actual methods, to be developed in $\S 3$, are all connected with a very important circumstance, which has so far not been clear: the $n$-dimensional level surface $J_{1}=a_{1}, \ldots, J_{n}=a_{n}$, when continued into the complex domain, is an Abelian variety (the complex torus $T^{2 n}$ ), which is the Jacobian variety of the Riemann surface $\Gamma$ given by the equation $R(W, E)=0$ in which $R(W, E)=\operatorname{det}(W-\Lambda)=W^{2}+P_{2 n+1}(E)$. This important property generalizes the natural case $n=1$, where the Abelian varieties are defined as the complex solutions of the equation $u^{\prime \prime}=\frac{\partial P_{3}(u)}{\partial u}, P_{3}$ being a polynomial of degree 3 . The corresponding result when the number of lacunae is $n>1$ is non-trivial and will be obtained by combining the methods of $\S 3$ with those of the present section.

We give one more useful application of the equations (2.1.15) and (2.2.17) for the matrices $\hat{T}$ and $\Lambda$. We recall the formula (2.1.19) for $\chi_{R}(x, E)$ (in the base (2.1.1)), $\chi_{R}(X, E)=\frac{k \sqrt{1-a_{R}^{2}}}{a_{\mathrm{Im}}+b_{\mathrm{Im}}}$. From (2.1.5) and (2.1.17) follow the general identities 


$$
\left\{\begin{array}{l}
2 \mu_{R}=-\frac{d}{d x_{0}}\left(\frac{\lambda_{\text {Im }}+\mu_{\text {Im }}}{k}\right), \\
2 b_{R}=-\frac{d}{d x_{0}}\left(\frac{a_{\mathrm{Im}}+b_{\mathrm{Im}}}{k}\right) .
\end{array}\right.
$$

Comparing these with the form of $\chi_{R}$ and the condition $\dot{a}_{R} \equiv 0$, we obtain

$$
\dot{\chi}_{R}=\left(\alpha \chi_{R}\right)^{\prime}, \text { where } \quad \alpha=\frac{\lambda_{\operatorname{Im}}+\mu_{\operatorname{Im}}}{k} .
$$

For the K-dV equation we have $\Lambda=\Lambda_{1}$ and $\alpha=-2(2 E+u)$. From (2.2.41) it follows that the quantity $I(k)=p(E)=\int_{x_{0}}^{x_{0}+T} \chi_{R} d x=\int_{x_{0}}^{x_{0}+T} \chi d x$ is conserved. We shall use $(2.2 .41)$ in $\S 3$ in the calculation of the dynamics of finite-zone potentials in terms of the parameters $\gamma_{1}, \ldots, \gamma_{n}$. As $E \rightarrow \infty$, we have the expansion

$$
I(k) \sim k T+\sum_{n \geqslant 0} \frac{1}{(2 k)^{2 n+1}} \int_{x_{0}}^{x_{0}+T} \chi_{2 n+1}(x) d x,
$$

where the integrals $I_{n-1}=\int_{x_{0}}^{x_{0}+T} \chi_{2 n+1} d x$ are Hamiltonians of the higher order K-dV equations, $\dot{u}=\frac{\partial}{\partial x} \frac{\delta I_{n}}{\delta u(x)}, \dot{L}=\left[A_{n}, L\right]$. It is natural to introduce a generating function for this set of equations. We consider an operator $A_{z}$, which depends on the parameter $z$ and has the following property (by (2.1.17) and (2.1.15)):

$$
\left[A_{z}, L\right]=\frac{\partial}{\partial x} \frac{\delta p(z)}{\delta u(x)}=\frac{\partial}{\partial x}\left(-\frac{1}{2 \chi_{R}(x, z)}\right),
$$

where $\chi(x, z)=\chi_{R}+i \chi_{I}, \chi_{I}=\frac{1}{2}\left(\ln \chi_{R}\right)^{\prime},-i \chi^{\prime}+\chi^{2}+u-E=0$. Then all the operators $A_{n}$ are obtained as coefficients of the expansion of $A_{z}$, as $z \rightarrow \infty$, in powers of $z^{-\frac{1}{2}}$. We can verify that $A_{z}$ has the form

$$
\text { (2.2.43) } \quad A_{z}=-\frac{1}{4}\left[\frac{1}{\chi_{R}(x, z)} \frac{d}{d x}-\frac{1}{2}\left(\frac{1}{\chi_{R}(x, z)}\right)^{\prime}\right] \frac{1}{L_{-2}} .
$$

We now give a convenient algorithm for obtaining all the matrices $\Lambda_{m}$ (in the base (2.1.2)), which will also be very useful in Ch. 3 for the generalization to first order matrix operators. We consider the differential equation $\lambda^{\prime}=[Q, \lambda]$, in which the matrix $Q$ in the base (2.1.2) has the form $Q=\left(\begin{array}{cc}0 & E-u \\ -1 & 0\end{array}\right)$. This equation has a unique solution as a formal 
series $\lambda=\left(\begin{array}{ll}0 & 1 \\ 0 & 0\end{array}\right)+\frac{\lambda_{1}}{E}+\frac{\lambda_{2}}{E^{2}}+\ldots ;$ the coefficients $\lambda_{n}$ are determined from the recurrence relation $\left[\lambda_{n},\left(\begin{array}{ll}0 & 1 \\ 0 & 0\end{array}\right)\right]=\lambda_{n-1}^{\prime}+\left[\lambda_{n-1},\left(\begin{array}{ll}0 & 0 \\ 1 & 0\end{array}\right)\right]$. Then the matrices $\Lambda_{m}$ have the form

$$
\Lambda_{m}=\left(\begin{array}{ll}
0 & 1 \\
0 & 0
\end{array}\right) E^{m}+\lambda_{1} E^{m-1}+\ldots+\lambda_{m}
$$

§3. The inverse problem for periodic and almost-periodic (real and complex) finite-zone potentials. The connection with the theory of Abelian varieties

Starting from the Definitions 1 and 1 ' of Ch. 2, $\S 1$, by a "finite-zone" potential we now understand a potential $u(x)$ for which the Bloch eigenfunction $\psi_{ \pm}\left(x, x_{0}, E\right)$, normalized by (2.1.6) and (2.1.7), is meromorphic on the Riemann surface $\Gamma$ :

$$
\Gamma: W^{2}=P_{2 n+1}(E)=\prod_{i=1}^{2 n+1}\left(E-E_{i}\right)
$$

and has the asymptotic form

$$
\psi_{ \pm} \sim e^{ \pm i k\left(x-x_{0}\right)}, \quad k^{2}=E, \quad E \rightarrow \infty .
$$

According to (2.1.13), we study on $\Gamma$ the function $\chi_{R}$,

$$
\chi_{R}=\frac{1}{2 i} W\left(\psi_{+}, \psi_{-}\right)=\frac{1}{2 i}\left(\psi_{+}^{\prime} \psi_{-}-\psi_{-}^{\prime} \psi_{+}\right),
$$

using the representation (2.1.14) and (2.1.16), (2.1.16'):

$$
\psi_{ \pm}\left(x, x_{0}, E\right)=\sqrt{\frac{\chi_{R}\left(x_{0}, E\right)}{\chi_{R}(x, E)}} \exp \left\{i \int_{x_{n}}^{x} \chi_{R}(x, E) d x\right\},
$$

where according to (2.1.19) $\chi_{R}=\frac{k \sqrt{1-a_{R}^{2}}}{a_{\mathrm{I}}+b_{\mathrm{I}}}$ (basis (2.1.1), real $k$ ) or $\chi_{R}=\sqrt{1-\frac{1}{4}\left(\alpha_{11}+\alpha_{22}\right)^{2}} / \alpha_{21}$ (basis (2.1.2), arbitrary $E$ ). We note that at degenerate points $E_{n}$ of the spectrum we have, according to (2.1.10), $\hat{T}= \pm\left(\begin{array}{ll}1 & 0 \\ 0 & 1\end{array}\right), \alpha_{21}=0$, and $\alpha_{21}\left(x_{0}, E\right)$ has a simple zero in $E$. By contrast, at non-degenerate points $E_{n}$ of the spectrum we have $\alpha_{21}\left(x_{0}, E_{n}\right) \not \equiv 0$. Let $\widetilde{E}_{\alpha}(\alpha=1,2,3, \ldots)$ be all the degenerate points of the (periodic and antiperiodic) spectrum. Since the quantity $\left(1-a_{R}^{2}\right)=1-\frac{1}{4}\left(\alpha_{11}+\alpha_{22}\right)^{2}$ has a two-fold zero at all points $\widetilde{E}_{\alpha}$ and a simple zero at the non-degenerate points $E_{1}, \ldots, E_{2 n+1}$, we see that

$$
\sqrt{1-a_{R}^{2}}=\sqrt{\prod_{i=1}^{2 n+1}\left(E-E_{i}\right)} \cdot f(E)
$$

where $f(E)$ is an entire function having only simple zeros at the points $\widetilde{E}_{\alpha}$. 
Therefore, the quotient

$$
\tilde{\alpha}_{21}=\frac{\alpha_{21}}{f(E)}
$$

is an entire function of $E$. We have the expression

$$
\chi_{R}=\frac{\sqrt{\prod_{i=1}^{2 n+1}\left(E-E_{i}\right)}}{\widetilde{\alpha}_{21}} .
$$

As $E \rightarrow \infty$, the quantity $\chi_{R}\left(x_{0}, E\right)$ has the asymptotic form $\chi_{R} \sim k=\sqrt{E}$. Hence, by $(2.3 .5)$, the quantity $\widetilde{\alpha}_{21}$ has the asymptotic form

$$
\widetilde{\alpha}_{21} \sim E^{n} \quad \text { as } \quad E \rightarrow \infty \text {. }
$$

Since $\alpha_{21}\left(x_{0}, E\right)$ is an entire function, we see from (2.3.6) that it is, in fact, a polynomial:

$$
\widetilde{\alpha}_{21}\left(x_{0}, E\right)=\prod_{j=1}^{n}\left(E-\gamma_{j}(x)\right)
$$

From this we obtain the final result.

COROLLARY 3. The quantity $x_{R}\left(x_{0}, E\right)=\frac{1}{2 i} W\left(\psi_{+}, \psi_{-}\right)$has the following form:

$$
\chi_{R}(x, E)=\frac{\sqrt{\prod_{i=1}^{2 n+1}\left(E-E_{i}\right)}}{\prod_{j=1}^{n}\left(E-\gamma_{j}(x)\right)}=\frac{\sqrt{P(E)}}{P_{n}(x, E)} .
$$

NOTE. The quantity $\widetilde{\alpha}_{21}=\prod_{j=1}^{n}\left(E-\gamma_{j}(x)\right)$ can be determined otherwise;

it is a solution of the equation

$$
-y^{m \prime}+4(u-E) y^{\prime}+2 u^{\prime} y=0 \text {. }
$$

which is polynomial in $E$.

From this argument we can obtain another derivation of (2.3.8), since (2.3.8) is satisfied by the product $\varphi_{1} \cdot \varphi_{2}$ of two solutions of the equation $L \varphi_{\alpha}=E \varphi_{\alpha}$, setting $\varphi_{1}=\psi_{+}, \varphi_{2}=\psi_{-}$(the derivation in [41] and [47]).

Following the derivation in [40] and [46] we evaluate the product $\psi_{+} \psi_{-}$starting from (2.1.14):

$$
\psi_{+} \psi_{-}=\frac{\chi_{R}\left(x_{0}, E\right)}{\chi_{R}(x, E)}=\prod_{j=1}^{n} \frac{E-\gamma_{j}(x)}{E-\gamma_{j}\left(x_{0}\right)} .
$$

Formally speaking, this derivation refers only to periodic potentials because it uses the translation matrix $\hat{T}$. However, the formulae (2.3.8) and (2.3.10) hold also in the almost-periodic case. To prove this we can proceed as follows: later, when we obtain the functions $\gamma_{j}(x)$, we have to verify that the function $\psi_{ \pm}\left(x, x_{0}, E\right)$ defined by $(2.1 .14)$ really satisfies the 
equation $L \varphi=E \varphi$.

We now draw some conclusions from the results obtained.

1. The poles of the Bloch function lie only on one sheet over the points $\gamma_{j}\left(x_{0}\right)$ if all the $\gamma_{j}\left(x_{0}\right)$ are pairwise distinct.

PROOF. From (2.1.16), (2.3.8) we see that the poles of $\psi_{ \pm}$can lie only above points $\gamma_{j}\left(x_{0}\right)$ where $\chi_{R}$ has poles, since $\psi_{ \pm}=c+i \chi\left(x_{0}, E\right) s$ and since $c$ and $s$ are entire functions of $E$. If there were poles on both sheets $\left(\gamma_{j}\left(x_{0}\right), \pm\right)$ then in the product $\psi_{+} \psi_{-}=\prod_{j} \frac{E-\gamma_{j}(x)}{E-\gamma_{j}\left(x_{0}\right)}$ we would have a double pole. This contradiction completes the proof. We note that for real periodic potentials the poles $\gamma_{j}\left(x_{0}\right)$ occur one each in the forbidden zones.

2. Symmetric functions of $\gamma_{1}(x), \ldots, \gamma_{n}(x)$ can be expressed in terms of the potential $u(x)$ and its derivatives; in fact,

$$
\begin{gathered}
u(x)=-2 \sum_{j=1}^{n} \gamma_{j}(x)+\sum_{i=1}^{2 n+1} E_{i}, \\
\sum \gamma_{i} \gamma_{j}=\frac{1}{8}\left(3 u^{2}-u^{n}\right)+\frac{1}{2} \sum E_{i} E_{j}-\frac{3}{8}\left(\sum E_{i}\right)^{2} .
\end{gathered}
$$

PROOF. Starting from the asymptotic form of $\chi_{R}(x, k)$ as $k \rightarrow \infty$, we have (see (2.1.18)

$$
\chi_{R}(x, k) \sim k+\sum_{n \geqslant 1} \frac{\chi_{2 n+1}(x)}{(2 k)^{2 n+1}},
$$

where $\chi_{1}(x)=-u(x), \ldots$ Comparing this fact with the form of $\chi_{R}$ (see (2.3.8)), we obtain the result.

3. Each finite-zone potential $u(x)$ is a stationary solution of one of the higher order $\mathrm{K}-\mathrm{dV}$ equations (2.2.22).

PROOF. We start from (2.1.17):

$$
\frac{\delta p(E)}{\delta u(x)}=\frac{\delta}{\delta u(x)} \int_{x_{0}}^{x_{0}+T} \chi_{R}(x, E) d x=-\frac{1}{2 \chi_{R}(x, E)}
$$

in the case of periodic potentials. From the form of $\chi_{R}$ it follows that amongst the coefficients of the expansion of $-\frac{1}{2 \chi_{R}}=\frac{\delta p}{\delta u}$ in powers of $\frac{1}{\sqrt{E}}$ there are only finitely many linearly independent functions of $x$, and this leads to the relation

$$
\sum_{m=-1}^{n} c_{m} \frac{\delta I_{m}}{\delta u(x)}=0
$$

where $I_{m-1}=\int_{x_{0}}^{x_{0}+T} x_{2 m+1} d x(m \geqslant 0)$ are the coefficients of the expansion 
of $p(E)$, as $E \rightarrow \infty$, as follows from (2.1.18) and (2.1.15). From this the proof follows in the periodic case. The almost-periodic case is similar: the integrals over the period must be replaced by the mean value; all the formulae remain valid. For real periodic functions the result is suggested by standard uniqueness theorems (see [1]), from which it follows that the potentials form at most an $n$-dimensional family for a given spectrum (with $n$ forbidden zones); actually, by the results of $\mathrm{Ch} .2, \S 2$ we have an exactly $n$-dimensional family. However, this result is needed also for almostperiodic and for complex potentials.

4. The set of quantities $\gamma_{1}(x), \ldots, \gamma_{n}(x)$ satisfies the system of equations

$$
\gamma_{j}^{\prime}= \pm \frac{2 i \sqrt{P_{2 n+1}\left(\gamma_{j}\right)}}{\prod_{k \neq j}\left(\gamma_{h}-\gamma_{j}\right)} .
$$

Strictly speaking, these equations must be treated as equations in $x_{0}$ for the set of points $P_{1}\left(x_{0}\right), \ldots, P_{n}\left(x_{0}\right)$ on $\Gamma$, where $P_{j}\left(x_{0}\right)=\left(\gamma_{j}\left(x_{0}\right), \pm\right)$, and the poles of $\psi_{ \pm}\left(x, x_{0}, E\right)$ lie at $P_{j}\left(x_{0}\right)$. Above each lacuna $\left[E_{2 i}, E_{2 i+1}\right]=l_{i}$ in the real case lies the cycle $a_{i}$ on $\Gamma$ that is obtained by fusing the two intervals $a_{i}=\left(l_{i},+\right) \cup\left(l_{i},-\right)$ at the end-points $\left(E_{2 i},+\right)=\left(E_{2 i},-\right)$ and $\left(E_{2 i+1},+\right)=\left(E_{2 i+1},-\right)$. The points $P_{j}\left(x_{0}\right)$ lie one each on the cycles $a_{j}$ (by Corollary 1 above) and more over these cycles as $x_{0}$ varies. The whole "phase point" $\left(P_{1}, \ldots, P_{n}\right)$ lies on the torus $T^{n}=S_{1}^{1} \times \ldots \times S_{n}^{1}$ (the direct product of the cycles $a_{j}$ ), and (2.3.12) is actually written down for the motion of the point $\left(P_{1}, \ldots, P_{n}\right) \in T^{n}$.

PROOF OF (2.3.12). According to 1. above, the poles of $\psi_{ \pm}$lie only on one of the sheets $\left(\gamma_{j}, \pm\right)$ over $\gamma_{j}\left(x_{0}\right)$. On the other sheet there is no pole at $\left(\gamma_{j}, \pm\right)$. This means that in the formula $(2.1 .16)$ for $\psi_{ \pm}$, or what is the same thing, in the quantity $\chi=\chi_{R}+i \chi_{I}=\chi_{R}+\frac{i}{2} \frac{d}{d x_{0}} \ln \chi_{R}$ (by (2.1.12)) the pole is cancelled for $E=\gamma_{j}$ and one choice of sign in the radical. From the form (2.3.8) of $\chi_{R}$ we obtain

$$
\left(\frac{d}{d x} \frac{i}{2} \prod_{k=1}^{n}\left(E-\gamma_{k}\right)\right)_{E=\gamma_{j}}=\left(\sqrt{\prod_{k=1}^{2 n+1}\left(E-E_{k}\right)}\right)_{E=\gamma_{j}} .
$$

Solving (2.1.13) for $\gamma_{j}^{\prime}$ we obtain (2.3.12). This proves the assertion.

We now analyse by way of illustration cases of one and two zones; the two-zone potentials were not previously known.

EXAMPLE 1. $(n=1)$. The equations (2.3.12) have the form

$$
\gamma=\gamma_{1}, \quad \gamma^{\prime}= \pm 2 i \sqrt{\prod_{j=1}^{3}\left(\gamma-E_{j}\right)}, \quad u=-2 \gamma+\sum_{i=1}^{3} E_{i}
$$

these formulae transform in an obvious way into (2.2.35).

EXAMPLE 2. $(n=2)$. The equations $(2.3 .12)$ for the parameters have the form 


$$
\gamma_{1}^{\prime}= \pm \frac{2 i \sqrt{P_{5}\left(\gamma_{1}\right)}}{\gamma_{1}-\gamma_{2}}, \quad \gamma_{2}^{\prime}= \pm \frac{2 i \sqrt{P_{5}\left(\gamma_{2}\right)}}{\gamma_{2}-\gamma_{1}}
$$

where $-2\left(\gamma_{1}+\gamma_{2}\right)=u, \gamma_{1} \gamma_{2}=\frac{1}{8}\left(3 u^{2}-u^{\prime \prime}\right)+\frac{1}{2} \Sigma E_{i} E_{j}$. As was indicated in $\S 2$ (see (2.2.39), $\gamma_{1}$ and $\gamma_{2}$ are coordinates on the level surface of the two integrals $J_{1}$ and $J_{2}$ for the stationary solutions of the second order K-dV equation.

The equations (2.3.14) can be integrated by a change of variable

$$
d \tau=\frac{1}{\gamma_{2}-\gamma_{1}} d x
$$

For real potentials, where $E_{2} \leqslant \gamma_{1} \leqslant E_{3}, E_{4} \leqslant \gamma_{2} \leqslant E_{5}$, we introduce two functions $F_{1}(\tau)$ and $F_{2}(\tau)$, setting

$$
\tau=\int_{F_{2}}^{F_{1}} \frac{d q}{2 \sqrt{P_{5}(q)}}, \quad \tau=\int_{E_{4}}^{F_{2}} \frac{d q}{2 \sqrt{P_{5}(q)}} .
$$

We select an initial point $\tau_{0}$ so that

$$
\gamma_{1}(\tau)=F_{1}(\tau), \quad \gamma_{2}(\tau)=F_{2}\left(\tau+\tau_{0}\right) .
$$

From (2.3.15) we have

$$
x-x_{0}=\int_{0}^{\tau}\left(F_{2}\left(q+\tau_{0}\right)-F_{1}(q)\right) d q .
$$

The potential $u(x)$ has the form $u(x)=-2\left(\gamma_{1}+\gamma_{2}\right)+\sum_{i=1}^{5} E_{i}$, and in conjunction with (2.3.16), (2.3.17) and (2.3.18) we obtain our final expression for the two-zone almost periodic potentials:

where

$$
u(x)=-2\left[F_{1}(\tau)+F_{2}\left(\tau+\tau_{0}\right)\right]+\sum_{i=1}^{5} E_{i},
$$

$$
x-x_{0}=\int_{0}^{\tau}\left(F_{2}\left(q+\tau_{0}\right)-F_{1}(q)\right) d q .
$$

In (2.2.38) values of the boundaries of the zones $E_{i}$ were exhibited for which, under a special choice of $x_{0}$ and $\tau_{0}$, the Lamé potential $3 u(x)$ results, in which $u(x)$ is a one-zone potential (the Weierstrass 8 -function). Other potentials of this family (with the same spectrum as $3 u(x)$ ) have the form (see [42])

$$
\left\{\begin{array}{c}
v(x)=28\left(x-\beta_{1}\right)+28\left(x-\beta_{2}\right)+2 \wp\left(x-\beta_{3}\right), \\
\beta_{1}+\beta_{2}+\beta_{3}=0, \\
\beta_{2}-\beta_{3}=\frac{1}{2} \wp^{-1}\left[-\wp^{-1}\left(\beta_{1}-\beta_{3}\right)+\sqrt{g_{2}-3 \wp^{(02}\left(\beta_{1}-\beta_{3}\right)}\right] .
\end{array}\right.
$$

We now pass on to the connection with Abelian varieties. We have proved (see (2.3.11)) the formula $u(x)=-2 \Sigma \gamma_{j}+2 \Sigma E_{i}$ for the potential 
$u(x)$. The Bloch eigenfunction $\psi_{ \pm}\left(x, x_{0}, E\right)$ has the set of poles $P_{1}\left(x_{0}\right), \ldots, P_{n}\left(x_{0}\right)$, which are points on the Riemann surface $\Gamma$, situated one each over the points $\gamma_{1}\left(x_{0}\right), \ldots, \gamma_{n}\left(x_{0}\right)$ of the $E$-plane. Strictly speaking, (2.3.11) is to be understood as follows: there is a canonical projection $\pi: \Gamma \rightarrow \mathrm{C}$ of $\Gamma$ onto the $E$-plane, and the potential $u(x)$ has the form

$$
u(x)=-2 \sum_{j=1}^{n} \pi\left(P_{j}(x)\right)+\text { const. }
$$

Essentially, the projection $\pi$ is a numerical function on $\Gamma$, invariant under interchange of the sheets (the canonical involution, which always occur on a hyperelliptic surface $\Gamma$ ). It is natural to define a numerical function $\sigma$ on the symmetrized set of $n$ points $\left(P_{1}, \ldots, P_{n}\right)$ such that

$$
\sigma\left(P_{1}, \ldots, P_{n}\right)=\sum_{j=1}^{n} \pi\left(P_{j}\right) .
$$

The symmetrized sets $\left(P_{1}, \ldots, P_{n}\right)$ of points of $\Gamma$ form an algebraic variety, the symmetric power $S^{n}(\Gamma)$ ), and $\sigma$ is an algebraic function on this variety. The set of points $\left(P_{1}, \ldots, P_{n}\right)$ varies when the parameter $x$ is changed, and the value of the function

$$
-2 \sigma\left(P_{1}(x), \ldots, P_{n}(x)\right)+\text { const }
$$

is the potential $u(x)$, by (2.3.11). In classical algebraic geometry it has long been known that the symmetric power is birationally isomorphic to the $2 n$-dimensional torus $\mathbf{J}(\Gamma)$, the Jacobian variety for $\Gamma$. Therefore, in particular, $\sigma$ is expressible in terms of multidimensional $\theta$-functions (of the Riemann $\theta$-function and its derivatives). The birational equivalence $S^{n}(\Gamma) \rightarrow \mathbf{J}(\Gamma)$ is realized by the standard Abel map, which we describe below. We select a base of cycles on $\Gamma$ :

$$
a_{1}, \ldots, a_{n}, \ldots, b_{1}, \ldots, b_{n},
$$

where the intersection matrix has the form

$$
a_{i} \circ a_{j}=b_{i} \circ b_{j}=0, \quad a_{i} \circ b_{j}=\delta_{i j}
$$

(the cycles $a_{i}$ for a real hyperelliptic surface $\Gamma$ were indicated above, they are the complete inverse images of the forbidden zones on $\Gamma$ ). We consider the basis of holomorphic differentials on $\Gamma$ :

$$
\Omega_{k}=\sum_{l=1}^{n} c_{k l} \frac{E^{l-1} d E}{\sqrt{P_{2 n+1}(E)}} \quad(k=1, \ldots, n)
$$

normalized by the conditions

So we obtain the matrix

$$
\oint_{a_{j}} \Omega_{k}=2 \pi i \delta_{j k} .
$$

$$
B_{k l}==\oint_{b_{l}} \Omega_{k} .
$$


We know that this matrix is symmetric and has a real part of definite sign (the matrix $B_{k l}$ cannot be split into blocks, for $n=2$ it is a complete set of constraints). The full $n \times 2 n$ matrix

$$
\left(\oint_{a_{j}} \Omega_{k}, \oint_{b_{j}} \Omega_{k}\right)=\left(2 \pi i \delta_{j k}, B_{j k}\right)
$$

defines $2 n$ vectors in $n$-dimensional complex space $\mathbf{C}^{n}$, the integral linear combinations of which form a lattice that defines the torus $J(\Gamma)$ as a Jacobian variety (the "Jacobian of $\Gamma$ "). The Abel map $A: \mathbf{S}^{n}(\Gamma) \rightarrow \mathbf{J}(\Gamma)$ is defined as follows. We fix the points $P_{1}^{0}, \ldots, P_{n}^{0}$. We set

$$
A\left(P_{1}, ., P_{n}\right)=\left(\eta_{1}, \ldots, \eta_{n}\right),
$$

where

$$
\eta_{k}=\sum_{j=1}^{n} \int_{P_{j}^{0}}^{P_{j}} \Omega_{k},
$$

and $\Omega_{k}$ is the basis (2.3.22) of holomorphic differentials. Clearly, the parameters $\eta_{k}$ are determined to within a vector of the lattice (2.3.24). This is the way the Abel mapping is constructed.

An important observation, mainly due to Akhiezer [10] consists in the fact that (translating the language of [10] into contemporary language) under the Abel map the set of zeros $\left\{P_{1}(x), \ldots, P_{n}(x)\right\}$ of the eigenfunction $\psi_{ \pm}$transforms into a straight line on the Jacobian variety (we shall show later that the eigenfunction $\mathscr{E}$, which Akhiezer constructed in his examples for the half-line is the same as the Bloch function $\psi_{ \pm}\left(x, x_{0}, E\right)$ when the potential is even, $u(x)=u(-x)$, and $\left.x_{0}=0\right)$. We use the information on the zeros $P_{j}(x)$ of $\psi_{ \pm}\left(x, x_{0}, E\right)$ lying over the points $\gamma_{j}(x)$, on the poles $P_{j}\left(x_{0}\right)$ lying over $\gamma_{j}\left(x_{0}\right)$, and on the asymptotic form $\psi_{ \pm} \sim e^{ \pm i k\left(x-x_{0}\right)}$, as $E \rightarrow \infty$. We consider the logarithmic differential

$$
\omega=\left(\frac{d \ln \psi}{d E}\right) d E,
$$

which has the following properties:

a) it has poles with residue -1 at the points $P_{j}\left(x_{0}\right)$;

b) it has poles with residue +1 at the points $P_{j}(x)$;

c) it has a pole of the second order at $E=\infty$, which in the local parameter $z$ has the form: $\omega \sim-i\left(x-x_{0}\right) \frac{d z}{z^{2}}$, where $z=\frac{1}{k}=\frac{1}{\sqrt{E}}$, since $\psi_{ \pm} \sim e^{ \pm i k\left(x-x_{0}\right)}$, as $E \rightarrow \infty$;

d) all the integrals over the cycles $\oint_{a_{i}} \omega$ and $\oint_{b_{i}} \omega$ are integer multiples of $2 \pi i$, because $\psi_{ \pm}$is a single-valued function on $\Gamma$.

We claim that the properties a), b), c), d) completely determine the 
function $\psi_{ \pm}\left(x, x_{0}, E\right)$ and that as $x$ varies, the set of zeros $\left(P_{1}(x), \ldots, P_{n}(x)\right)$ moves in a straight line on the Jacobian variety $\mathrm{J}(\Gamma)$ after the Abel map A.

PROOF. We introduce the differential $\Omega$ whose only singularity is a pole of the second order at $E=\infty$, and whose asymptotic form is $\Omega=-i \frac{d z}{z^{2}}$, as $E \rightarrow \infty$, where $z=\frac{1}{\sqrt{E}}$. We normalize $\Omega$ by the conditions

Then $\Omega$ has the form

$$
\oint_{a_{j}} \Omega=0 \quad(j=1, \ldots, n) \bullet
$$

$$
\Omega=i \frac{E^{n}+\alpha_{1} E^{n-1}+\ldots+\alpha_{n}}{2 \sqrt{P_{2 n+1}(E)}} d E
$$

where the coefficients $\alpha_{j}$ are determined by (2.3.26).

Let $\Omega^{P Q}$ be a differential on $\Gamma$ whose only singularities are a pole of residue -1 at $Q$ and a pole of residue +1 at $P$, normalized by the condition

$$
\oint_{a_{j}} \Omega^{P Q}=0 \quad(j=1, \ldots, n) .
$$

In algebraic geometry it is known that if the $\Omega_{j}$ are normalized by $\left(2.3 .22^{\prime}\right)$ and $\Omega^{P Q}$ by (2.3.27), then (for any path from $P$ to $Q$ )

$$
\oint_{b_{j}} \Omega^{P Q}=\int_{Q}^{P} \Omega_{j},
$$

where the cycles $\left(a_{i}, b_{j}\right)$ satisfy $\left(2.3 .21^{\prime}\right)$. We now consider the differential $\omega=d_{E} \ln \psi$ and represent it in the form

$$
\omega=\left(x-x_{0}\right) \Omega+\sum_{j=1}^{n} \Omega^{P_{j}(x) P_{j}\left(x_{0}\right)}+D,
$$

where $D$ is the holomorphic differential $D=\sum_{i=1}^{n} \alpha_{i} \Omega_{i}$. From the condition $\oint_{a_{j}} \omega=2 \pi i m_{j}$; in which the $m_{j}$ are integers, we obtain, using the

normalizations (2.3.22), (2.3.26) and (2.3.27),

$$
2 \pi i m_{j}=\left(\sum_{k=1}^{n} \alpha_{k} \delta_{k j}\right) 2 \pi i \quad(j=1, \ldots, n),
$$

from which it follows that all the $\alpha_{j}$ are integers, $\alpha_{j}=m_{j}$. From the conditions $\oint_{b_{j}} \omega=2 \pi i n_{j}$, in which $n_{j}$ are the integers, we obtain, using (2.3.28)

(2.3.30) $2 \pi i n_{j}=\left(x-x_{0}\right) U_{j}+\sum_{k=1}^{n} \int_{P_{k}\left(x_{0}\right)}^{P_{h}(x)} \Omega_{j}+\sum_{k=1}^{n} m_{k} B_{k j}$, where $U_{j}=-\oint_{b_{j}} \Omega$. From (2.3.30) it also follows that, to within a lattice 
vector, under variation of the parameter the set of points $\left(Q_{1}(x), \ldots, Q_{n}(x)\right)$, describes a straight line on the torus $\mathbf{J}(\Gamma)$ whose gradient is defined by the vector $\left(U_{1}, \ldots, U_{n}\right)$, depending only on $\Gamma$.

(2.3.30) can be rewritten as

$$
\eta_{j}=\eta_{j}^{0}+\left(x-x_{0}\right) U_{j},
$$

in which the $n_{j}$ are coordinates in $\mathrm{C}^{n}$, defined to within an element of the lattice (2.3.24). The restriction of the algebraic function $\sigma$ to the rectilinear winding $\left(2.3 .30^{\prime}\right)$ also gives (in the real case) the potential $u(x)$, which is almost periodic with the group of periods $\left(T_{1}, \ldots, T_{n}\right)$, where

$$
T_{j}^{-1}=\sum_{k=1}^{n} B^{j k} U_{k} \text {. }
$$

(Here $B^{j k}$ is the inverse of the matrix of periods (2.3.23).) Under continuation into the complex $x$-domain, the potential becomes a meromorphic almost-periodic function with $2 n$ periods $T_{1}, \ldots, T_{n}, T_{1}^{\prime}, \ldots, T_{n}^{\prime}$, where the $T_{j}^{\prime}$ along the imaginary axis have the form

$$
T_{j}^{\prime}=\frac{2 \pi i}{U_{j}} \text {. }
$$

For complex $\Gamma$ the potential has $2 n$ periods in the complex domain. For the potential $u(x)$ to be periodic in $x$, it is necessary and sufficient that the real periods $T_{1}, \ldots, T_{n}$ satisfy $n-1$ equations of the form

$$
\sum_{j=1}^{n} n_{i j} T_{j}=0 \quad(i=1, \ldots, n-1),
$$

where the $n_{i j}$ are integers.

If the whole group of $2 n$ complex periods $\left(T_{1}, \ldots, T_{n}, T_{1}^{\prime}, \ldots, T_{n}^{\prime}\right)$ reduces to two generators, then $u(x)$ is expressible in terms of elliptic functions (for this $2 n-2$ integral linear relations are needed).

Thus, the Abel map integrates the equations (2.3.12) for the quantities $\left(\gamma_{1}, \ldots, \gamma_{n}\right)$. From our results so far, bearing in mind that $u(x)$ is uniquely determined by the set of initial points $\left[P_{1}\left(x_{0}\right), \ldots, P_{n}\left(x_{0}\right)\right]$ on $\Gamma$, we obtain the following theorem.

THEOREM 1) The set of (real and complex) almost periodic finite-zone potentials with a given spectrum $\Gamma$ is canonically isomorphic to the Jacobian variety $\mathbf{J}(\Gamma)$ of $\Gamma$, which is a 2 -dimensional Abelian variety, and this isomorphism is realized by the analytic operations described above. The group of periods is determined by the spectrum $\Gamma$. 2) The set of all complex solutions of the stationary problem for higher order $K-d V$ equations is, to within a birational equivalence, for given constants $\left(d, c_{1}, \ldots, c_{n}\right)$ and levels of the commuting integrals $J_{1}, \ldots, J_{n}$ (or for a given spectrum) the Abelian variety $\mathbf{J}(\Gamma)$, where the Riemann surface $\Gamma$ is defined by the equation $W^{2}-P_{2 n+1}(E)=0$ (see (2.2.24)). The affine part of $\mathrm{J}(\Gamma)$ is canonically embedded in the space $\mathrm{C}^{2 n}$.

As was shown above (see (2.3.20)), $u(x)$ has the form 


$$
u(x)=-2 \sigma\left(\eta_{1}^{0}+\left(x-x_{0}\right) U_{1}, \ldots, \eta_{n}^{0}+\left(x-x_{0}\right) U_{n}\right)+\text { const, }
$$

in which $\sigma$ is an algebraic function on the torus $\mathbf{J}(\Gamma)$ and is expressed in algebraic form in terms of the Riemann $\theta$-function and its derivatives, where the classical Riemann $\theta$-function is constructed from the lattice (2.3.24) in the standard form:

(2.3.33) $\theta\left(\eta_{1}, \ldots, \eta_{n}\right)=\sum_{m_{1}, \ldots, m_{n}} \exp \left\{\frac{1}{2} \sum_{j, k} B_{j k} m_{j} m_{k}+\sum_{k} m_{k} \eta_{k}\right\}$ (the $m_{1}, \ldots, m_{n}$ are integers).

In [41] there is a convenient explicit formula expressing $\sigma$ in terms of the $\theta$-function (2.3.33). For $u(x)$ we have

(2.3.34) $u(x)=$

$$
\begin{gathered}
=-2 \frac{d^{2}}{d x^{2}} \ln \theta\left(\eta_{1}^{0}+U_{1}\left(x-x_{0}\right)-K_{1}, \ldots, \eta_{n}^{0}+U_{n}\left(x-x_{0}\right)-K_{n}\right)+C, \\
K_{\mathbf{j}}=\frac{1}{2} \sum_{k=1}^{n} B_{k j}-\pi i j, \quad \eta_{j}^{0}=\sum_{k} \int_{\infty}^{P_{k}\left(x_{0}\right)} \S_{j} ;
\end{gathered}
$$

where $C$ is a constant depending only on $\Gamma$. Let us prove this formula. We consider the function

$$
F(P)=\theta\left(\eta(P)-\eta^{0}\right),
$$

where $P \in \Gamma$ and $\eta_{j}(P)=\int_{\infty}^{P} \Omega_{j}$. The function $F(P)$ is single-valued on $\widetilde{\Gamma}$ cut along the cycles $\left(a_{j}, b_{j}\right)$ (2.3.21). By a result of Riemann, (see [56]), $F(P)$ has $n$ zeros $P_{1}, \ldots, P_{n}$ on $\Gamma$ (for almost all $\eta^{0} \in \mathbf{J}(\Gamma)$, and the following relation holds on $\mathbf{J}(\Gamma)$ :

$$
A\left(P_{1}, \ldots, P_{n}\right)=\eta^{0}-\vec{K},
$$

where $K_{j}=\frac{1}{2} \sum_{k=1}^{n} B_{k j}-\pi_{i j}$ are the "Riemann constants". Therefore,

$$
\begin{aligned}
& \frac{1}{2 \pi i} \oint_{\partial \widetilde{\Gamma}} \pi_{j}(P) d \ln F(P)=\sum_{j=1}^{n} \pi\left(P_{j}\right)+ \\
& \quad+\underset{P=\infty}{\operatorname{res}} \pi(P) d \ln F(P)=\sigma\left(\eta^{0}-\vec{K}\right)+\underset{P=\infty}{\operatorname{res}} \pi(P) d \ln F(P) .
\end{aligned}
$$

Since the $\theta$-function (2.3.33) has the properties

$$
\begin{gathered}
\theta\left(\eta_{1}, \ldots, \eta_{k}+2 \pi i, \ldots, \eta_{n}\right)=\theta\left(\eta_{1}, \ldots, \eta_{n}\right)_{,} \\
\theta\left(\eta_{1}+B_{1 k}, \ldots, \eta_{n}+B_{n k}\right)=\exp \left(-\frac{B_{k k^{\top}}}{2}-\eta_{k}\right) \theta\left(\eta_{1}, \ldots, \eta_{n}\right),
\end{gathered}
$$

the integral (2.3.37) is

$$
\frac{1}{2 \pi i} \oint_{\partial \widetilde{\Gamma}} \pi(P) d \ln F(P)=\sum_{k=1}^{n} \oint_{a_{k}} \pi(P) \omega_{k} .
$$


From (2.3.37) and (2.3.37') we have

$$
\sigma\left(\eta^{0}-\vec{K}\right)=-\operatorname{res} \pi(P) d \ln F+\sum_{k=1}^{n} \oint_{i_{k}} \pi(P) \omega_{k} .
$$

To derive (2.3.34) from (2.3.38) we must calculate the residue res $\pi(P) d \ln F$ at $P=\infty$, where $\eta^{0}$ is chosen so that

$$
\left.\eta^{0}-\vec{K}=A\left(P_{1}(x), \ldots, P_{n}(x)\right)=A\left(p_{1}\left(x_{0}\right), \ldots P_{n}\left(x_{0}\right)\right)+\vec{U} x-x_{0}\right) .
$$

To do this it remains to observe that the quantities $U_{j}$ in (2.3.30) and $c_{k l}$ in (2.3.22) are connected by $U_{j}=c_{j n}$ (a consequence of the relation between the periods of the differentials on $\Gamma$; see [55]).

From the preceding results we have seen that the Bloch function $\psi_{ \pm}$and the potential $u$ itself are completely determined by the spectrum, (the Riemann surface $\Gamma$ ), and the "divisor" (the set of poles), that is, the numbers $\gamma_{1}\left(x_{0}\right), \ldots, \gamma_{n}\left(x_{0}\right)$ together with an indication of the sheet on which the poles lie. The zeros of $\psi_{ \pm}$also form a divisor $\left(\gamma_{1}(x), \sigma_{1}\right),\left(\gamma_{2}(x), \sigma_{2}\right), \ldots,\left(\gamma_{n}(x), \sigma_{n}\right)$, where $\sigma_{j}= \pm$ labels the sheet in which the zero lies. If the potential is real, then all the $\gamma_{j}$ lie in the lacunae $E_{2 j} \leqslant \gamma_{j} \leqslant E_{2 j+1}$, the Riemann surface is real, and it makes sense to talk of the "upper sheet" $(+)$ and the "lower sheet" $(-)$ for real values of $E$ over the lacunae (the positive and negative values of the root $\left.\sqrt{-P_{2 n+1}}\right)$. If the sign of the sheet is + , then $\psi_{+}\left(x, x_{0}, E\right) \rightarrow 0$, as $x \rightarrow+\infty$, for $E$ inside the lacuna; if the sign of the sheet is - when $E$ is in the lacuna, then $\psi_{-}\left(x, x_{0}, E\right) \rightarrow 0$, as $x \rightarrow-\infty$. Hence the function $\psi_{ \pm}\left(\tau, x_{0}, E\right)=f_{j}(\tau), E=\gamma_{j}(x)$, belongs to the discrete (non-degenerate) spectrum of the Sturm-Liouville problem on the half-line

$$
\begin{array}{ll}
\tau \geqslant x, & f(x)=0, \quad f(+\infty)=0, \\
\tau \leqslant x, & f(x)=0, \quad f(-\infty)=0,
\end{array}
$$

depending on the sign $( \pm)$ of the sheet on which the zero $P_{j}(x)$ of $\psi\left(x, x_{0}, E\right)$ lies, and the set $\left\{\gamma_{j}\right\}$ comprises all the non-degenerate levels of these spectra. A similar though rather simpler situation arose in the work of Shabat [11]: he suggested a method of studying non-reflecting potentials by means of "conditional" eigenvalues $\gamma_{j}$ on two half-lines for which he derived in [11] an equation analogous to (2.3.5) (but, of course, in this case the Riemann surface degenerates and there is, in general, no Abelian variety; equations of the type (2.3.5) in this degenerate case were derived by completely different methods). In our case, when $x$ varies, the set of points $\left(\gamma_{j}, \pm\right)$ describes the cycles $a_{j}$, and the sign of the sheet changes when passing through a branch-point.

We consider now the case of even potentials, $u(x)=u(-x)$, with $x_{0}=0$. It is easy to verify that when $x \mapsto-x$ the sheets of $\Gamma$ are exchanged. Hence, for the equation $u(x)=u(-x)$ to hold it is necessary and sufficient that 
the poles $\gamma_{j}(0)$ are invariant under this exchange, that is, occur at branchpoints. Moreover, when $x_{0}=0$, the function $\chi\left(x_{0}, E\right)$ has the form

since

$$
\chi(0, E)=\chi_{R}(0, E)
$$

$$
\chi_{I}(0, E)=-\frac{1}{2}\left[\frac{d}{d x} \ln \prod_{j=1}^{n}\left(E-\gamma_{j}\right)\right]_{x=0}=0,
$$

because $\gamma_{j}^{\prime}(0)=0$; this follows from (2.3.5) and the fact that $\gamma_{j}(0)$ lies at a branch-point. Therefore, $\psi_{ \pm}(x, 0, E)$ in this case has the form

$$
\psi_{ \pm}(x, 0, E)=c+i \frac{\sqrt{P_{2 n+1}(E)}}{\prod_{j=1}^{n}\left(E-\gamma_{j}(0)\right)} s,
$$

where the $\gamma_{j}(0)$ lie at the branch-points (ends of the lacunae). This gives the Akhiezer formula of [10] for the function $\mathscr{E}$ in case the $\gamma_{j}(0)$ are taken as the lower boundaries of the lacunae. The point of the derivation is the fact that for a given zone structure there are only finitely many even potentials.

We conclude this section by showing that the parameters $\left(\eta_{1}, \ldots, \eta_{n}\right)$ on the torus $\mathbf{J}(\Gamma)$ give "angle" variables, canonically conjugate to the "action" variables, which, for the Hamiltonian systems (2.2.22), can be taken as the integrals $J_{1}, \ldots, J_{n}$, the last $n$ coefficients of the polynomial $P_{2 n+1}=\operatorname{det} \Lambda$ (see $\left.\S 2\right)$. The Poisson brackets here form the constant nonsingular matrix

$$
\left\{\begin{array}{l}
{\left[J_{k}, \eta_{j}\right]=a_{k j}} \\
{\left[J_{k}, J_{s}\right]=\left[\eta_{k}, \eta_{s}\right]=0 .}
\end{array}\right.
$$

It turns out (see $\S 4$ ) that the parameters $\eta_{k}$ on $\mathbf{J}(\Gamma)$ also give the set of "angles" for the time evolution by the K-dV and higher order K-dV equations: by the higher order $\mathrm{K}-\mathrm{dV}$ equations, all the derivatives $\dot{\eta}_{k}$ are constant, and by virtue of the operator $\frac{\partial}{\partial x}$, which is connected with the time dynamics, the Poisson brackets all vanish: $\left[\eta_{k}, \eta_{s}\right]=0$.

§4. Applications. The time dynamics of finite-zone potentials according to the K-dV equations. The universal fibering of Jacobian varieties (the hyperelliptic case).

We study the time evolution according to any of the higher order K-dV equations. In Ch. $2, \S 2$ we have derived the equation (2.2.33) for the time evolution, which for $m=1$ coincides with the usual K-dV equation:

$$
\frac{d \Lambda}{d t_{m}}=\left[\Lambda, \Lambda_{m}\right]
$$

where $\Lambda=\sum_{i=0}^{n} c_{i} \Lambda_{n-i},(2.4 .1)$ holds for the set of solutions of (2.2.22) 


$$
\sum c_{i} \frac{\delta I_{n-i}}{\delta u(x)}=d
$$

and the algorithm for finding the matrices in the basis (2.1.2) is given by (2.2.44).

Starting from the results of $\$ 3$, we now find formulae for the time dynamics in terms of the parameters $\gamma_{j}$. We use (2.2.41), which in the basis (2.1.1) has the form $\frac{\partial \chi_{R}}{\partial t}=\frac{\partial}{\partial x}\left(\alpha \chi_{R}\right)$, where $\alpha=\frac{\lambda_{I}+\mu_{I}}{k}$ and $\alpha=-2(u+2 E)$ for the initial $\mathrm{K}-\mathrm{dV}$ equation $(m=1)$. Using the form

$$
\chi_{R}=\frac{\sqrt{P_{2 n+1}(E)}}{P_{n}(E, x)},
$$

we obtain

$$
\frac{\partial P_{n}}{\partial t}=\frac{\partial \alpha}{\partial x} P_{n}-\alpha \frac{\partial P_{n}}{\partial x}
$$

or, substituting $E=\gamma_{j}$ in (2.4.2) after solving for $\dot{\gamma}_{j}$ and using (2.3.5) for $\gamma_{j}^{\prime}$

$$
\dot{\gamma}_{j}= \pm \frac{2 i \alpha_{j} \sqrt{P_{2 n+1}\left(\gamma_{j}\right)}}{\prod_{k \neq j}\left(\gamma_{k}-\gamma_{j}\right)}
$$

where $\alpha_{j}=-\left.2(u+2 E)\right|_{E=\gamma_{j}(x)}$ for the original $\mathrm{K}-\mathrm{dV}$ equation, and by (2.3.11) $u=-2\left(\sum_{j=1}^{n} \gamma_{j}\right)+\sum_{i=1}^{2 n+1} E_{i}$; this gives the final formula for $\dot{\gamma}_{j}$.

EXAMPLE. Consider $n=2$ and the original $\mathrm{K}-\mathrm{dV}$ equation with $\alpha=-2(u+2 E)$. We obtain

$$
\left\{\begin{array}{l}
\dot{\gamma}_{1}= \pm \frac{8 i\left(\gamma_{2}-\frac{1}{2} \sum_{i=1}^{5} E_{i}\right) \sqrt{P_{5}\left(\gamma_{1}\right)}}{\gamma_{1}-\gamma_{2}} \\
\dot{\gamma}_{2}= \pm \frac{8 i\left(\gamma_{1}-\frac{1}{2} \sum_{i=1}^{5} E_{i}\right) \sqrt{P_{5}\left(\gamma_{2}\right)}}{\gamma_{2}-\gamma_{1}}
\end{array}\right.
$$

As in (2.3.15), we introduce the parameter $\tau$ given by $d \tau=\frac{d x}{\gamma_{2}-\gamma_{1}}$, and obtain

$$
\left\{\begin{array}{l}
\frac{d \gamma_{1}}{\sqrt{-P_{5}\left(\gamma_{1}\right)}}= \pm 8\left(\gamma_{2}-\frac{1}{2} \sum E_{i}\right) d \tau \\
\frac{d \gamma_{2}}{\sqrt{-P_{5}\left(\gamma_{2}\right)}}= \pm 8\left(\gamma_{1}-\frac{1}{2} \sum E_{i}\right) d \tau .
\end{array}\right.
$$

Suppose, for simplicity, that $\sum_{i=1}^{5} E_{i}=0$. Introducing the parameter $w$ where $8\left(\gamma_{2} \gamma_{1}\right) d \tau=d w$, we then have 


$$
\frac{\gamma_{1} d \gamma_{1}}{\sqrt{-P_{5}\left(\gamma_{1}\right)}}=d w, \quad \frac{\gamma_{2} d \gamma_{2}}{\sqrt{-P_{5}\left(\gamma_{2}\right)}}=d w
$$

where $d w$ is a first order differential on $\Gamma$. Now (2.4.4) and thus also (2.4.3) can be integrated in the obvious way. Comparing the result with the formulae (2.3.11) for the potential, we find that $\frac{\partial}{\partial t} \tau_{0}=4, \frac{\partial}{\partial t} x_{0}=4\left(F_{1}\left(\tau_{0}\right)-\frac{1}{2} \Sigma E_{i}\right)$, and the potential has the form

$$
\text { (2.4.5) } u(x, t)=-2\left[F_{1}\left(\tau\left(x-x_{0}(t)\right)\right)+F_{2}\left(\tau\left(x-x_{0}(t)\right)+\tau_{0}(t)\right)\right]+\Sigma E_{i},
$$

where the hyperelliptic functions $F_{1}$ and $F_{2}$ are defined in $\mathrm{Ch} .2, \S 3$ (see (2.3.16).

If $u(x, 0)$ is the Ince (Lamé) potential, where $u(x, 0)=68(x)$ with the boundaries of the zones $(2.2 .38)\left(\wp^{\circ}(x)\right.$ is the Weierstrass elliptic function), then we have

$$
u(x, t)=2 \wp\left(x-\beta_{1}(t)\right)+2 \wp\left(x-\beta_{2}(t)\right)+2 \wp\left(x-\beta_{3}(t)\right),
$$

where

$$
\begin{gathered}
\beta_{1}+\beta_{2}+\beta_{3}=0, \quad t=\int_{0}^{\beta_{1}-\beta_{2}} \frac{d z}{\sqrt{12\left(g_{2}-3 \xi^{2}(z)\right)}}, \\
\beta_{2}-\beta_{3}=\frac{1}{2} \wp^{-1}\left[-\wp\left(\beta_{1}-\beta_{3}\right)+\sqrt{g_{2}-3 \wp^{2}\left(\beta_{1}-\beta_{3}\right)}\right] .
\end{gathered}
$$

It turns out that the Abel map (2.3.25) integrates (2.4.3) for $\mathrm{K}-\mathrm{dV}$ equation and all its higher order analogues, and the parameters $\eta_{k}$ have constant derivatives by virtue of all these equations. We can calculate the derivatives

$$
\dot{\eta}_{k}=W_{k}^{(m)}
$$

by the $m$ th K-dV equation:

$$
\frac{\partial}{\partial t} u=\frac{\partial}{\partial x} \frac{\delta I_{m}}{\delta u(x)} .
$$

The idea of this calculation, by analogy with $\$ 3$, consists in the fact that under time development the eigenfunction $\psi_{ \pm}\left(x, x_{0}, E\right)$ has the asymptotic form, as $E \rightarrow \infty$ (for a suitable normalization)

$$
\Psi \sim \exp \left[i k\left(x-x_{0}\right)+i k^{2 n+1}\left(t-t_{0}\right)\right] .
$$

If (2.4.9) can be established (this involves certain difficulties), then, by analogy with $\S 3$, we obtain the following result: let $\omega_{m}$ be a differential on $\Gamma$ such that at $E=\infty$ it has a pole of order $2 m+2$ (for the $m$ th $\mathrm{K}-\mathrm{dV}$ equation $(2.4 .8)$, as $E \rightarrow \infty$ )

$$
\omega_{m}=\frac{d z}{z^{2 m+2}}+\text { a regular part }
$$

where $z=-\frac{1}{\sqrt{E}}=\frac{1}{k}$, and normalized by the conditions 
$\left(2.4 .10^{\prime}\right)$

$$
\oint_{\substack{a_{j} \\ \omega_{m}}} \omega_{m} \quad(j=1, \ldots, n) .
$$

Arguing as in $\S 3$ in "the case of the differential $d_{E} \ln \psi$, we have the required result, namely

$$
\eta_{k}=W_{k}^{(m)}, \quad W_{k}^{(m)}=\oint_{b_{k}} \omega_{m} .
$$

Substituting in the formula (2.3.34), which expresses the potential $u(x, t)$ in terms of the Riemann $\theta$-function, we obtain the $\mathrm{K}-\mathrm{dV}$ dynamics in the form

$$
\text { (2.4.12) } \begin{aligned}
u(x, t)= & -2 \frac{d^{2}}{d x^{2}} \ln \theta\left(\left(x-x_{0}\right) U_{1}+\left(t-t_{0}\right) W_{1}^{(1)}+\right. \\
& \left.+\eta_{1}^{0}-K_{1}, \ldots,\left(x-x_{0}\right) U_{n}+\left(t-t_{0}\right) W_{n}^{(1)}+\eta_{n}^{0}-K_{n}\right)+C .
\end{aligned}
$$

So we have obtained the following result.

THEOREM. The time development of finite-zone potentials according to the $\mathrm{K}-\mathrm{dV}$ equation or any of its higher order analogues is described by (2.4.3), (2.4.5), (2.4.11), (2.4.12) and represents a motion on a torus along a rectilinear winding on the variety of all potentials with the given spectrum $\Gamma$, which is isomorphic to the Jacobian variety $\mathrm{J}(\Gamma)$, the complex torus $\mathrm{T}^{2 n}$. In particular, the rectilinear structure on $\mathrm{J}(\Gamma)$ is determined by "higher order K-dV" equations, written in the form of a set of commuting polynomial Hamiltonian systems with $n$ degrees of freedom and the Hamiltonians $J_{1}, \ldots, J_{n}$ in the realization of $\S 2$.

In the case of a real Riemann surface $\Gamma$ (or a real bounded potential) the motion is on the torus $\mathbf{T}^{n}$, which can be visualized in the form of a direct product of the cycles $a_{j}$, the inverse images of the lacunae on $\Gamma$. This motion is conditionally periodic in time with a set of $n$ real and $n$ imaginary periods, where the periods are expressed in terms of the integrals $W_{k}^{(m)}$ of the differentials $\omega_{m}$ (for the $m$ th analogue of the $\mathrm{K}-\mathrm{dV}$ equation) over the cycles $b_{k}$ and the lattice matrix $B_{j l}$.

Since the higher order $\mathrm{K}-\mathrm{dV}$ equations are rectilinear on $\mathbf{J}(\Gamma)$, we see that on this Abelian variety the law of addition of points, by means of motions along these systems holds. In the realization of $\S 2$ of the varieties $J(\Gamma)$ of finite-zone potentials by the equations $J_{1}=J_{1}^{0}, \ldots, J_{n}=J_{n}^{0}$, all the higher order $\mathrm{K}-\mathrm{dV}$ equations were realized as Hamiltonian dynamical systems in a phase space with $n$ degrees of freedom, depending on the $n+1$ constants $\left(d, c_{1}, \ldots, c_{n}\right)$ (if $\Sigma E_{i}=0$, then $\left.c_{1}=0\right)$, and the Hamiltonians $J_{\alpha}(\alpha=1, \ldots, n)$ of these systems are polynomials in the phase variables, having vanishing Poisson brackets $\left[J_{\alpha}, J_{\beta}\right]=0$. The Jacobian $\mathbf{J}(\Gamma)$ is defined in $\mathrm{C}^{2 n}$ by the equations $J_{1}=J_{1}^{0}, \ldots, J_{n}=J_{n}^{0}$, and the Riemann surface $\Gamma$ has the form

$$
R(W, E)=W^{2}-P_{2^{n+1}}=0,
$$


where

$$
P_{2 n+1}(E)=-\operatorname{det} \Lambda, \Lambda=\Lambda_{n}+\sum_{i=1}^{n} c_{i} \Lambda_{n-i}
$$

for $n=2$, according to (2.2.36), we have the Abelian varieties $\mathbf{J}(\Gamma)$ :

$$
\begin{gathered}
J_{1}=p_{1} p_{2}-\left(\frac{1}{2} q_{2}^{2}+\frac{5}{2} q_{1}^{2} q_{2}+\frac{5}{8} q_{1}^{4}\right)+c_{2} q_{1}^{2}-d q_{1}, \\
J_{2}=p_{1}^{2}-2 q_{1} p_{1} p_{2}+2\left(q_{2}-c_{2}\right) p_{2}^{2}+q_{1}^{5}+2 c_{2} q_{1}^{3}+d q_{1}^{2}-4 q_{1} q_{2}^{2}+4 c_{2} q_{1} q_{2}-2 d q_{2} ; \\
-\operatorname{det} \Lambda=P_{5}(E)=E^{5}+\frac{1}{4} c_{2} E^{3}-\frac{1}{16} d E^{2}+\left(-\frac{1}{32} J_{1}+\frac{1}{4} c_{2}^{2}\right) E+J_{2} / 2^{8}+c_{2} d / 2^{7},
\end{gathered}
$$

where $c_{1}=\Sigma E_{i}=0, q_{1}=u, q_{2}=-\frac{5}{2} u^{2}+u^{\prime \prime}, p_{1}=q_{2}^{\prime}, p_{2}=u^{\prime}$.

Isomorphic Abelian varieties are obtained when the Riemann surfaces are isomorphic, that is, when the roots $\left(E_{i}\right)$ of the polynomial $P_{2 n+1}(E)$ differ only by a factor $\lambda$ if $\Sigma E_{i}=c_{1}=0$. After taking account of this equivalence, there remain altogether $2 n+2$ isomorphic surfaces $\Gamma$ ( $n$ being the genus) because one of the $2 n+2$ branch-points is distinguished and is situated at $E=\infty$. We recall that hyperelliptic Riemann surfaces are characterized uniquely by the roots of a polynomial $Q_{2 n+2}(E)$ to within a common fractionally-linear transformation and an arbitrary permutation of the roots. We place one branch-point $E_{2 n+2}=\infty$ at infinity, and also assume that $\Sigma E_{i}=0$; the remaining $2 n$ numbers can be simultaneously multiplied by one and the same number $\lambda$. Furthermore, all the coefficients of the polynomial det $\Lambda=P_{2 n+1}(E)$ can be expressed symmetrically in terms of $E_{1}, \ldots, E_{2 n+1}$. The only remaining non-symmetry in our constructions is the selection of the branch-points $E_{2 n+2}=\infty$.

Let $v \in \mathbf{V}$ be a base point of the variety of moduli of hyperelliptic curves $\Gamma$ defined by the set $v=\left(E_{1}, \ldots, E_{2 n+2}\right)$ to within a common fractionally-linear transformation and a permutation; let $\widetilde{\mathbf{V}} \stackrel{2 n+2}{\rightarrow} \mathrm{V}$ be our covering associated with the selection of the branch-point $E_{2 n+2}=\infty$. Over each point of $\mathrm{V}$ there is the Jacobian variety $\mathbf{J}(\Gamma)$ of the corresponding curve $\Gamma$, and we have the universal fibering of Jacobian varieties $\stackrel{\mathbf{J}(\Gamma)}{\rightarrow} \mathbf{V}$ and its $(2 n+2)$-sheeted covering $\widetilde{\mathbf{M}} \stackrel{\mathbf{J}(\Gamma)}{\rightarrow} \widetilde{\mathbf{V}}$, where $\widetilde{v} \in \widetilde{\mathbf{V}}$ is determined by the set $\widetilde{v}=\left(E_{1}, \ldots, E_{2 n+1}\right)$ to within a permutation and multiplication: $\left(E_{1}, \ldots, E_{2 n+1}\right) \sim \lambda\left(E_{1}, \ldots, E_{2 n+1}\right), \Sigma E_{i}=0$. This variety $\widetilde{\mathbf{M}}$ can be computed in our constructions as follows: in $\mathbf{C}^{3 n}$ the coordinates are $z_{1}, \ldots, z_{3 n}$, where $z_{i}=q_{i}, z_{n+i}=p_{i}, i \leqslant n ; z_{2 n+1}=d, z_{2 n+i}=c_{i}, 2 \leqslant i \leqslant n$, and the symplectic form $\Omega=\sum_{i} d p_{i} \wedge d q_{i}$. All the Abelian varieties are given by the equations

$$
\left\{\begin{aligned}
z_{j} & =\text { const }, \quad j \geqslant 2 n+1, \\
J_{\alpha} & =\text { const }, \quad(\alpha=1, \ldots, n),
\end{aligned}\right.
$$


and thus lie in the spaces $\mathbf{C}^{2 n}$. The universal fibering splits into a family of fiberings of each $\mathrm{C}^{2 n}\left\{z_{j}=\right.$ const, $\left.j>2 n\right\}$ by level surfaces of all the polynomials $J_{\alpha}$, depending on the remaining $n$ coordinates $z_{2 n+1}, \ldots, z_{3 n}$ and on the parameters. An algorithm for calculating the polynomials $J_{\alpha}$ was described in $\S 2$. The law of addition on the Abelian varieties and all the one-parameter subgroups are given by Hamiltonian systems with the Hamiltonians $J_{\alpha}$. The group of multiplications of the roots $E_{i}$ by $\lambda$ acts by multiplying the coefficients of the polynomial $P_{2 n+1}(E)=\operatorname{det} \Lambda$ by the corresponding power of $\lambda$. We can choose $\lambda$ by requiring that $c_{2}=1$.

Thus, we obtain the following result:

The manifold $\widetilde{\mathbf{M}}$, the space of the universal fibering of the Jacobian varieties $\mathrm{J}(\Gamma)$ with the distinguished branch-point $E_{2 n+2}=\infty$, is a rational variety; this universal fibering with the fibre $\mathbf{J}(\Gamma)$ splits into a family of fiberings with rational fibering spaces of dimension $2 n$, fibred by the polynomials $J_{\alpha}$ (an algorithm to calculate them was given in \$2). On affine parts of the fibering space $\mathrm{C}^{2 n}$ there is a symplectic form, and the Poisson brackets of all pairs of polynomials $J_{\alpha}$ vanish. The Abelian varieties are complex solutions of this commuting set of Hamiltonian systems.

We mention that the variety $\mathbf{M}$ itself is probably also rational, but here we have only proved its universality. In the general (non-hyperelliptic) case we can also develop an analogous method, using operators of higher order instead of the Schrödinger operator. For an approach to these problems, see Ch. $3, \S 2$.

\section{CHAPTER 3}

\section{GENERALIZATIONS. DISCRETE SYSTEMS AND MATRIX OPERATORS OF FIRST ORDER}

\section{$\S 1$. The periodic problem for the Toda chain and the "K-dV difference equation"}

As we have said in Ch. 1, §5, Manakov [35] and Flaschka [33], [34] found an $L-A$ pair for the Toda chain, proved that the Henon integrals [32] are involutory and integrated completely the Cauchy problem for the Toda chain with rapidly decreasing initial conditions $c_{n} \rightarrow$ const, $v_{n} \rightarrow 0$, as $|n| \rightarrow \infty$, by the method of scattering theory. Furthermore, with the operator $L$ under the condition $v_{n} \equiv 0$ another physically interesting system is associated, the "difference K-dV equation", discovered in [35], for which Manakov first found the integrals and then integrated the system by the same method as in the rapidly decreasing case. Following unpublished work of S. P. Novikov, we consider here the periodic case for both these systems, by a method similar to that of $\mathrm{Ch}$. 2. The operator $L$ has the form indicated in $\mathrm{Ch} .1, \S 5$, and the equation $L \psi_{n}=E \psi_{n}$ is 


$$
\left(E-v_{n}\right) \psi_{n}=i \sqrt{c_{n}} \psi_{n-1}-i \sqrt{c_{n+1}} \psi_{n+1}
$$

After the change $\psi_{n} \mapsto i^{n} \psi_{n}$ we obtain

$$
\left(E-v_{n}\right) \psi_{n}=\sqrt{c_{n}} \psi_{n-1}+\sqrt{c_{n+1}} \psi_{n+1} .
$$

The Wronskian for the operator (3.1.1') has the form

$$
W_{n}(\varphi, \psi)=(-1)^{n} \sqrt{c_{n+1}}\left(\Psi_{n+1} \varphi_{n}-\psi_{n} \varphi_{n+1}\right)
$$

and does not depend on $n$. We use here an analogue $c\left(n, n_{0}, E\right), s\left(n, n_{0}, E\right)$ to the basis (2.1.2) of Ch. 2, where

$$
\begin{cases}c_{n_{0}}=1, & c_{n_{0}+1}=0, \\ s_{n_{0}}=0, & s_{n_{0}+1}=1,\end{cases}
$$

and $W(c, s)=\sqrt{c_{n_{0}+1}}(-1)^{n_{0}+1}$. In the periodic case the matrix of a translation by a period is defined in the usual way, (in the basis 3.1.2):

$$
\hat{T}=\left(\begin{array}{ll}
\alpha_{11} & \alpha_{12} \\
\alpha_{21} & \alpha_{22}
\end{array}\right)
$$

where the $\alpha_{i j}$ are real for real $E$ and $\operatorname{det} \hat{T}=1$ (we assume that the period $N$ is even). In exactly the same way the Bloch eigenfunctions $\psi_{ \pm}\left(n, n_{0}, E\right)$ are defined where $\psi_{n_{0}}=1, \hat{T} \psi_{ \pm}=e^{ \pm i p(E)} \psi_{ \pm}$; they are meromorphic on the Riemann surface $\Gamma$ and have branch-points at the boundaries of the zones. It is important to note that the operator $L$ (see (3.1.1) and (3.1.1') has altogether finitely many forbidden and solution zones, and the neighbourhood of $E=\infty$ is always a forbidden zone. Thus, $\Gamma$ is always of finite genus and is defined by the equation

$$
y^{2}=P_{2 n+2}(E)=\sum_{i=1}^{2 n+2}\left(E-E_{i}\right),
$$

where the $E_{i}$ are the boundaries of the zones. We introduce the notation:

$$
\left\{\begin{aligned}
\Psi_{ \pm}\left(n, n_{0}, E\right) & =\exp \left(\sum_{n_{0}}^{n-1} \Delta_{n}\right), \\
\chi^{ \pm}(n, E) & =e^{\Delta_{n}}
\end{aligned}\right.
$$

From (3.1.1) we obtain the following equation (an analogue to the Riccati equation):

$$
E-v_{n}=\sqrt{c_{n}} e^{-\Delta n-1}+\sqrt{c_{n+1}} e^{\Delta n} .
$$

In the solution zones, where $p(E)$ is real, we obtain from (3.1.6)

$$
\text { (3.1.7) }\left\{\begin{array}{l}
\chi=\chi_{R e}+i \chi_{\mathrm{Im}}, \quad \psi_{+}=\bar{\psi}_{-}, \\
\chi=\chi^{+}=\overline{\chi^{-}}, \quad \Delta_{n}=\Delta_{n_{R}}+i \Delta_{n_{I}}, \\
\Delta_{n_{R}}=-\frac{1}{2} \ln \left\{\left[\sqrt{c_{n+1}} \chi_{I_{m}}(n+1, E)\right]-\left[\sqrt{c_{n}} \chi_{\operatorname{Im}}(n, E)\right]\right\} .
\end{array}\right.
$$

From (3.1.7) it follows that (in the solution zones)

$$
\psi_{ \pm}\left(n, n_{\theta}, E\right)=\sqrt{\frac{\sqrt{c_{n+1}} \chi_{\operatorname{Im}}(n, E)}{\sqrt{c_{n}} \chi_{\operatorname{Im}}\left(n_{0}, E\right)}} \exp \left\{i \sum_{n_{0}}^{n-1} \Delta_{n_{I}}\right\} .
$$


Further, from the definition of $W$ we have

$$
\left\{\begin{array}{l}
W\left(\psi_{+}, \psi_{-}\right)=2 i \chi_{\operatorname{Im}}\left(n_{0}, E\right) \sqrt{c_{n_{0}+1}}, \\
\psi_{ \pm}=c+\chi\left(n_{0}, E\right) s, \quad \chi=\chi^{+} \quad \text { or } \quad \chi=\chi-
\end{array}\right.
$$

As in Ch. 2, the function $\chi\left(n_{0}, E\right)$ can be expressed in terms of $\hat{T}$, and $\chi_{\mathrm{Im}}(n, E)$ has the same form as in (2.1.19):

$$
\chi_{\operatorname{Im}}\left(n_{0}, E\right)=\frac{\sqrt{1-\frac{1}{4}(\mathrm{Sp} \hat{T})^{2}}}{\alpha_{21}} .
$$

As in Ch. 2, §3, the function $2 i \chi_{\operatorname{Im}}\left(n_{0}, E\right) \sqrt{c_{n_{0}+1}}$ can be continued from the solution zones to all complex values of $E$ by the formula (3.1.9) and coincides with the Wronskian $W\left(\psi_{+}, \psi_{-}\right)$. As $E \rightarrow \infty$, it has one of two asymptotic forms (we recall that the Riemann surface $\Gamma$ given by (3.1.4) has two sheets \pm over the point $E=\infty$, and $z=\frac{1}{E}$ is a local parameter on each of them)

$$
\left\{\begin{array}{l}
\chi^{+}(n, E) \rightarrow \frac{E}{\sqrt{c_{n+1}}}\left(1+O\left(\frac{1}{E}\right)\right), \\
\chi^{-}(n, E) \rightarrow \frac{\sqrt{c_{n+1}}}{E}\left(1+O\left(\frac{1}{E}\right)\right)
\end{array}\right.
$$

on the two sheets of $\Gamma$, as $E \rightarrow \infty$. Hence, for $W\left(\psi_{+}, \psi_{-}\right)=\chi^{+}-\chi^{-}$we have the asymptotic form, as $E \rightarrow \infty$ :

$$
W \rightarrow 2 E+O(1)
$$

Exactly as in Ch. 2, §3, from the asymptotic form (3.1.10') we obtain

$$
\frac{1}{2} W\left(\psi_{+}, \psi_{-}\right)=\frac{\sqrt{\prod_{i=1}^{2 m+2}\left(E-E_{i}\right)}}{\prod_{j=1}^{m}\left(E-\gamma_{j}\left(n_{0}\right)\right)} .
$$

From (3.1.11) it follows that in the solution zones

$$
\chi_{\operatorname{Im}}\left(n_{0}, E\right)=\frac{1}{2 \sqrt{c_{n_{0}+1}}} W\left(\psi_{+}, \psi_{-}\right) .
$$

Comparing with the formulae (3.1.7), which are applicable within the solution zones, we obtain

$$
\chi^{ \pm}(n, E)=\frac{\sqrt{R}+\sqrt{R+4 \Pi \Pi_{n} \Pi_{n+1} c_{n+1}}}{2 \sqrt{c_{n+1}} \Pi_{n}},
$$

where $R(E)=\prod_{j=1}^{2 m+2}\left(E-E_{i}\right), \Pi_{n}=\prod_{j=1}^{m}\left(E-\gamma_{j}(n)\right)$. However, this expression cannot yet be regarded as the final result for $\chi^{ \pm}\left(n_{0}, E\right)$. In fact, formally speaking (3.1.12) is algebraic in a Riemann surface $\Gamma^{\prime}$, covering $\Gamma$ doubly. We know that the quantity $\chi^{ \pm}\left(n_{0}, E\right)$ is algebraic on $\Gamma$ and has poles of first order over $\gamma_{j}\left(n_{0}\right)$ at only one of the inverse images of this point. This has an important consequence: the expression $R+4 c_{n+1} \Pi_{n} \Pi_{n+1}$ under the root sign must be a perfect square of some polynomial of degree $m+1$ : 


$$
R+4 c_{n+1} \Pi_{n} \Pi_{n+1}=\prod_{k=1}^{m+1}\left(E-\alpha_{k}(n)\right)^{2} .
$$

The conditions (3.1.13) lead to a full set of relations of the form

$$
\left\{\begin{aligned}
\gamma_{j}(n+1) & =f_{j}\left(\gamma_{1}(n), \ldots, \gamma_{m}(n)\right), \\
c_{n+1} & =c_{n+1}\left(\gamma_{1}(n), \ldots, \gamma_{m}(n)\right),
\end{aligned}\right.
$$

giving a difference analogue of the Dubrovin equations for the $\gamma_{j}(n)$, and an analogue, which we shall need, of the "trace identities" for expressing $c_{n+1}$ in terms of $\gamma_{1}(n), \ldots, \gamma_{m}(n)$.

Of course, from the asymptotic form of $\chi^{+}(n, E)$, as $E \rightarrow \infty$, we can obtain the usual trace identities as in Ch. 2; for from (3.1.6) we have

$$
\chi^{+}(n, E) \sim \frac{E}{\sqrt{c_{n+1}}}\left(1-\frac{v_{n}}{E}-\frac{c_{n}}{E^{2}}+O\left(\frac{1}{E^{3}}\right)\right) .
$$

Expanding (3.1.12), as $E \rightarrow \infty$, we obtain

$$
\left\{\begin{array}{l}
v_{n}=-\sum_{j=1}^{m} \gamma_{j}(n)+\frac{\sigma_{1}}{2}, \\
c_{n}+c_{n+1}+v_{n}^{2}-\frac{\sigma_{1} v_{n}}{2}=\sum_{j \neq k} \gamma_{j}(n) \gamma_{k}(n)+\frac{\sigma_{1}^{2}}{8}-\frac{\sigma_{2}}{2} .
\end{array}\right.
$$

Thus, the usual trace formulae are insufficient to calculate $c_{n+1}$, and we obtain the missing expressions from (3.1.13).

Here are the simplest examples.

$m=1$. For the sake of convenience, let $\sigma_{1}=\sum_{i=1}^{4} E_{i}=0$. Then from (3.1.13) we have

$$
\left\{\begin{array}{l}
\sigma_{2}+4 c_{n+1}=-2 \alpha_{n}^{2} \\
\sigma_{3}-4 c_{n+1}\left(\gamma_{1 n}+\gamma_{1 n+1}\right)=0 \\
\sigma_{4}+4 c_{n+1}\left(\gamma_{1 n} \gamma_{1 n+1}\right)=\alpha_{n}^{4} .
\end{array}\right.
$$

$m=2$. Let $v_{n} \equiv 0$ (symmetric spectrum). Then

$$
\begin{gathered}
R(E)=\prod_{i=1}^{3}\left(E+E_{i}\right)\left(E-E_{i}\right), \\
\gamma_{1},{ }_{n}+\gamma_{2, n} \equiv 0, \quad \sigma_{1}=\sigma_{3}=\sigma_{5}=0 .
\end{gathered}
$$

From (3.1.13) we obtain

$$
\left\{\begin{aligned}
\sigma_{4}-4 c_{n+1}\left(\gamma_{1 n}^{2}+\gamma_{1 n+1}^{2}\right) & =\frac{1}{4}\left(\sigma_{2}+4 c_{n+1}\right)^{2}, \\
4 c_{n+1} \gamma_{1 n}^{2} \gamma_{1 n+1}^{2} & =-\sigma_{6} .
\end{aligned}\right.
$$

In these examples we can easily obtain from (3.1.16) and (3.1.17) the Dubrovin equation and an expression for $c_{n}$.

Continuing to follow the scheme of $\mathrm{Ch} .2$, $\S 3$, we consider the analytic properties of the function $\psi_{ \pm}\left(n, n_{0}, E\right)$ on $\Gamma$ : 
1) $\psi_{ \pm}$has zeros $P_{j}(n)=\left[\gamma_{j}(n), \pm\right]$ and poles $P_{j}\left(n_{0}\right)=\left[\gamma_{j}\left(n_{0}\right), \pm\right]$, which for real $v_{n}$ and $c_{n}>0$ lie one each in the lacunae on one of the sheets of $\Gamma$.

2) As $E \rightarrow \infty$, we have on both sheets of $\Gamma$

$$
\psi_{ \pm} \sim E^{ \pm\left(n-n_{0}\right)} \cdot \text { const. }
$$

Hence the differential $d_{E} \ln \psi$ has poles of the first order with residue +1 at $P_{j}(n)$ and with residue -1 at $P_{j}\left(n_{0}\right)$; at $E=\infty$ it has poles with residues $\pm\left(n-n_{0}\right)$ on the sheets $( \pm)$. The integrals of $d_{E} \ln \psi$ over all the cycles are integer multiples of $2 \pi i$. If

$$
\Omega_{l}=\sum_{k=0}^{n-1} c_{k l} \frac{E^{k} d E}{\sqrt{R(E)}}
$$

are holomorphic differentials, normalized by the conditions

$$
\oint_{a_{j}} \Omega_{l}=2 \pi i \delta_{j l},
$$

where the $a_{j}$ are cycles over the lacunae, then by analogy with the arguments of $\$ 3$ we have

$$
\sum_{j=1}^{n} \int_{P_{j}\left(n_{0}\right)}^{P_{j}(n)} \Omega_{l}=\left(2 \pi i \oint_{b_{l}} \Omega\right)\left(n-n_{0}\right)
$$

where $\Omega=\Omega^{\infty_{+}, \infty_{-}}$is a differential having poles at the points $\left(\infty_{+}, \infty_{-}\right)$with residues $(+1,-1)$, respectively, normalized by the conditions $\underset{a_{j}}{\oint_{j}} \Omega=0$. Moreover, we have $\oint_{b_{j}} \Omega=\int_{\infty_{+}}^{\infty} \Omega_{j}$, as in $\S 3$, for the differentials $\Omega^{P Q}$, since the cycles $b_{j}$ are conjugate to $a_{j}$. In the real case we obtain

$$
\oint_{b_{j}} \Omega=2 \int_{E_{2 n+2}}^{\infty} \Omega_{j}=U_{j}
$$

From (3.1.18) and (3.1.19) we obtain, as in $\S 3$, using the trace identity (3.1.15), an analogue of the Matveev-Its formula for $v_{n}$ in terms of the restriction of the $\theta$-function to the rectilinear winding in the direction $U_{j}$ on the Jacobian variety $\mathrm{J}(\Gamma)$ :

$$
v_{n}=-\frac{d}{d n} \ln \frac{\theta\left(\left(n-n_{0}\right) \vec{U}+\eta_{+}^{0}-\vec{K}\right)}{\theta\left(\left(n-n_{0}\right) \vec{U}+\eta_{-}^{0}-\vec{K}\right)}+\text { const; } \quad\left(\eta_{ \pm}^{0}\right)_{j}=\sum_{k} \int_{\infty_{ \pm}}^{P_{k}^{\left(n_{0}\right)}} \Omega_{j} .
$$

Using the technique of Appendix 3, we can easily express the sum $c_{n}+c_{n+1}$ in terms of the Riemann $\theta$-function on the basis of the trace identities (3.1.15). However, we cannot obtain a convenient expression for 
$c_{n}$. In principle, we can perform all the calculations starting from (3.1.13). Incidentally, under the condition $v_{n} \equiv 0$ the spectrum of $L$ is symmetric:

$$
R(E)=\prod_{i=1}^{m+1}\left(E-E_{i}\right)\left(E+E_{i}\right) .
$$

The distribution of the zeros $\gamma_{j}(n)$ and the poles $\gamma_{j}\left(n_{0}\right)$ in the lacunae is also symmetric.

We now turn to the non-linear systems associated with $L$. Their Hamiltonians $I_{k}$ can be obtained, as indicated in [35], from the expansion of $\Delta_{n}(E)=\ln \chi(n, E)$ in terms of $E^{-1}$, as $E \rightarrow \infty$, which follows from (3.1.6) and (3.1.10):

$$
\begin{aligned}
\Delta_{n}^{+}(E) & \sim \ln E-\ln \sqrt{c_{n+1}}+\ln (1+O(1 / E))= \\
& =\ln \frac{E}{\sqrt{c_{n+1}}}+\sum_{q \geqslant 1} b_{q n} ! E^{q}= \\
& =\ln \left(E / \sqrt{c_{n+1}}\right)-\frac{v_{n}}{E}-\frac{c_{n}+\frac{v_{n}^{2}}{2}}{E^{2}}-\frac{\frac{v_{n}^{3}}{3}+v_{n} c_{n}+c_{n} v_{n-1}}{E^{3}}+\ldots
\end{aligned}
$$

All the integrals $I_{q}=\sum_{n=n_{0}}^{n_{0}+N} b_{q n}$ are conserved in time by each of the systems, and all these systems commute; by definition, $c_{n}=e^{x_{n}-x_{n-1}}$.

The canonical coordinates are $\left(x_{n}, v_{n}\right)$. The first of these systems has the form

$$
\left\{\begin{array}{lll}
\text { a) } \quad \dot{x}_{n}=1, \quad \dot{v}_{n}=0, & I_{1}=\sum v_{n} \\
\text { b) } \quad \dot{x}_{n}=v_{n}, \quad \dot{v}_{n}=c_{n+1}-c_{n}, \quad I_{2}=\sum\left(\frac{v_{n}^{2}}{2}+c_{n}\right) ; \\
\text { c) } \quad \dot{x}_{n}=v_{n}^{2}+c_{n+1}+c_{n}, \quad \dot{v}=\left(v_{n+1}+v_{n}\right) c_{n+1}-\left(v_{n}+v_{n-1}\right) c_{n}, \\
\end{array}\right.
$$

For the system $(3.1 .23), c)$ the variety $v_{n} \equiv 0$ is invariant, and on it the system has the form of the " $\mathrm{K}-\mathrm{dV}$ difference equation"

$$
\dot{c}_{n}=c_{n}\left(c_{n+1}-c_{n-1}\right) \text {. }
$$

To find important families of solutions of these equations we have to solve the stationary problem for linear combinations of these systems (that is, to look for the singular points of the Hamiltonians $H=\Sigma \lambda_{j} I_{j}$ ), which determines "potentials" $\left(v_{n}, c_{n}\right)$ with finitely many lacunae. In particular, the stationary solutions of the Toda chain (3.1.23), b) have the form of "0-zone" operators

$$
\left\{\begin{array}{lr}
v_{n}=\dot{x}_{n}=\text { const, } \quad H=I_{2}+\lambda I_{1} \\
\dot{v}_{n}=0=c_{n+1}-c_{n}
\end{array}\right.
$$

We obtain the "1-zone" operators $L$ from the stationary points of the Hamiltonian $H$, where 


$$
H=I_{3}+\lambda I_{2}+\mu I_{1}
$$

or

$$
\begin{aligned}
v_{n}^{2}+c_{n+1}+c_{n}+\lambda v_{n}+\mu & =0 \\
\left(v_{n+1}+v_{n}\right) c_{n+1}+\left(v_{n}+v_{n-1}\right) c_{n}+\lambda\left(c_{n+1}-c_{n}\right) & =0
\end{aligned}
$$

Clearly, the change of variables $v_{n} \rightarrow v_{n}+$ const, $c_{n} \rightarrow c_{n}$ allows us to assume that $\lambda \equiv 0$. However, (3.1.25) are difference equations and are difficult to solve (even in the one-lacuna case). To find an analytic form of the 1-zone operators $L$ we may proceed by one of two methods.

METHOD 1. We use the algebraic-geometric formula for $v_{n}$ (see (3.1.20) and then compute $c_{n}$, starting from (3.1.16).

METHOD 2. We use the fact that the time dynamics of the Toda chain for the one-zone potentials $\left(v_{n}, c_{n}\right)$ is a "simple wave" $\{v(n-c t), c(n-\alpha t)\}$ for all $t$, and we calculate the dynamics in $t$ instead of the difference equation in $n$.

After the Abel map, as in Ch. 3, $\S 4$, the time dynamics of all these systems becomes linear.

EXAMPLE 1. Let $m=1$; using (3.1.16) we obtain $\left(\Sigma E_{i}=\sigma_{1}=0\right)$

$$
\left\{\begin{array}{c}
\gamma_{n}+\gamma_{n+1}=\frac{\sigma_{3}}{L\left(\gamma_{n}\right)}\left[2 \gamma_{n}^{2}+\sigma_{2} \pm \sqrt{P_{4}\left(\gamma_{n}\right)} \mathrm{j},\right. \\
\gamma_{n}+\gamma_{n-1}=\frac{\sigma_{3}}{L\left(\gamma_{n}\right)}\left[2 \gamma_{n}^{2}+\sigma_{2} \mp \sqrt{P_{4}\left(\gamma_{n}\right)}\right] \\
P_{4}(E)=\prod_{i=1}^{4}\left(E-E_{i}\right), \quad L\left(\gamma_{n}\right)=4 \sigma_{4}-\sigma_{2}^{2}+4 \sigma_{3} \gamma_{n}, \\
\dot{v}_{n}=\dot{\gamma}_{n}=c_{n+1}-c_{n}=\frac{\sigma_{3}}{4}\left(\frac{1}{\gamma_{n+1}+\gamma_{n}}-\frac{1}{\gamma_{n}+\gamma_{n-1}}\right)= \\
=\sqrt{P_{4}\left(\gamma_{n}\right)}, \quad \sigma_{3} \neq 0 .
\end{array}\right.
$$

If $\sigma_{3}=0$, then we have

$$
\left\{\begin{array}{l}
\gamma_{n+1}+\gamma_{n}=0, \\
\dot{\gamma}_{n}=c_{n+1}-c_{n}=\sqrt{P_{4}\left(\gamma_{n}\right)} .
\end{array}\right.
$$

Thus, for $m=1$ the functions $\gamma_{n}(t)=\gamma(n-\alpha t)$ have the form of the elliptic function

$$
t-t_{0}=\int^{\gamma_{n}} \frac{d \tau}{\sqrt{P_{4}(\tau)}} .
$$

According to (3.1.16) we find for the coefficients $c_{n}$ and $v_{n}$

$$
\left\{\begin{array}{cc}
v_{n}=\gamma_{n} & \left(\sigma_{1}=0\right), \\
4 c_{n+1}=\frac{\sigma_{3}}{\gamma_{n}+\gamma_{n+1}} \quad\left(\sigma_{3} \neq 0\right), \\
\left(\sigma_{2}+4 c_{n+1}\right)^{2}+4 c_{n+1}-4 \sigma_{4} \gamma_{n}^{2}=0 \quad\left(\sigma_{3}=0\right),
\end{array}\right.
$$

where $\gamma_{n}+\gamma_{n+1}$ are determined from (3.1.26).

EXAMPLE 2. Let $m=2$ and $v_{n} \equiv 0$. 
We now consider the symmetric spectrum with $\sigma_{1}=\sigma_{3}=\sigma_{5}=0$. We have $\gamma_{1 n}+\gamma_{2 n}=0$ since $v_{n}=\Sigma \gamma_{j n} \equiv 0$; from the trace identities (3.1.15) we obtain

$$
-\left(c_{n+1}+c_{n}\right)=\gamma_{i n}^{2}+\frac{1}{2}\left(\sum E_{i}^{2}\right) .
$$

We denote $\gamma_{1 n}^{2}$ by $\gamma_{n}$. Using (3.1.17) we obtain

We now use the K-dV difference equation

$$
\left\{\begin{array}{c}
\gamma_{n}=\frac{t_{n}}{2} \pm \sqrt{\frac{P_{4}\left(c_{n}\right)}{16^{2} c_{n}^{2}}}, \\
\gamma_{n-1}=\frac{t_{n}}{2} \mp \sqrt{\frac{P_{4}\left(c_{n}\right)}{16^{2} c_{n}^{2}}}, \\
\gamma_{n}-\gamma_{n-1}=\frac{2}{16 c_{n}} \sqrt{P_{4}\left(c_{n}\right)}, \\
P_{4}(x)=\left[4 \sigma_{4}-\left(\sigma_{2}+4 x\right)^{2}\right]^{2}+16 \cdot 4 \sigma_{6} x \\
t_{n}=-\frac{\left(\sigma_{2}+4 c_{n}\right)^{2}}{16 c_{n}}+\frac{4 \sigma_{4}}{16 c_{n}} .
\end{array}\right.
$$

$$
c_{n}=c_{n}\left(c_{n+1}-c_{n-1}\right) \text {. }
$$

Together with (3.1.29) we have

$$
\left(\ln c_{n}^{*}\right)^{\cdot}=\gamma_{n}-\gamma_{n-1} \text {. }
$$

Using the expression for $\gamma_{n}$ and $\gamma_{n-1}$ in terms of $c_{n}$ we obtain

$$
\dot{c}_{n}=\frac{1}{8} \sqrt{P_{4}\left(c_{n}\right)} \text {, }
$$

where $P_{4}\left(c_{n}\right)=\left[4 \sigma_{4}-\left(\sigma_{2}+4 c_{n}\right)^{2}\right]^{2}+16 \cdot 4 \sigma_{6} c_{n}$.

Finally we note the difference analogues to certain other results of Ch. 2 .

By analogy with Ch. 2, $\S 2$, we define the matrices $Q\left(n_{0}, E\right)$ and $\Lambda\left(n_{0}, E\right)$ for a given non-linear system in the basis (3.1.3). Then

$$
\begin{gathered}
\hat{T}\left(n_{0}+1\right)=Q\left(n_{0}\right) \hat{T} Q^{-1}\left(n_{0}\right), \\
\frac{d}{d t} c\left(n, n_{0}, E\right)=\lambda_{11} c+\lambda_{12} s+A c, \\
\frac{d}{d t} s\left(n, n_{6}, E\right)=\lambda_{21} c+\lambda_{22} s+A s, \\
\dot{L}=[A, L], \quad \dot{T}=[\Lambda, T], \\
\Lambda=\left(\begin{array}{ll}
\lambda_{11} & \lambda_{12} \\
\lambda_{21} & \lambda_{22}
\end{array}\right), \quad Q=\left(\begin{array}{ll}
q_{11} & q_{12} \\
q_{21} & q_{22}
\end{array}\right) .
\end{gathered}
$$

The spectrum of $L$ such that $\dot{L}=[A, L]$ is defined on the Riemann surface $\Gamma$ :

$$
\operatorname{det}(y-\Lambda(E))=0,
$$

or, if $\operatorname{Sp} \Lambda=0$, then $y^{2}=\operatorname{det} \Lambda=P_{2 n+2}(E)$. As before, the coefficients of $\operatorname{det} \Lambda=P_{2 n+2}(E)$ give "integrals" of the difference equation for the stationary problem. They can be used, in particular, to embed the Jacobian variety $\mathbf{J}(\Gamma)$ in a projective space, by analogy with $\mathrm{Ch}$. 2 . From the compatibility condition for the equations 


$$
\dot{T}=[\Lambda, T] \text { and } T_{n+1}=Q T_{n} Q^{-1}
$$

we obtain

$$
\frac{d Q(n)}{d t}=\Lambda(n+1) Q(n)-Q(n) \Lambda(n) .
$$

These equations are equivalent to the original non-linear system. For the stationary problem, then

$$
\begin{aligned}
& \Lambda(n+1)=Q(n) \Lambda(n) Q^{-1}(n), \\
& \frac{d}{d t} Q(n)=0 .
\end{aligned}
$$

In conclusion we mention that our arguments require that $L$ is "local" (and that quantities of Wronskian kind are conserved). It is important for us that, irrespective of the value of the period, a basis of eigenfunctions can be determined (for a given $E$ ) by values in a set of neighbouring points, in which the normalization of the Bloch function $\psi_{ \pm}$can also be uniquely determined.

\section{$\S 2$. First order matrix operators and their associated non-linear systems}

We give here an account (with sketches of the proofs) of some very recent results of Dubrovin and Its as applied to non-linear systems associated with first order linear differential matrix operators. Its [52] has studied in detail the case of a two-dimensional matrix operator and its associated non-linear Schrödinger equation and modified $\mathrm{K}-\mathrm{dV}$ equation, by comparing trace formulae (see Appendix 3 of the present survey), and for this case he has obtained convenient explicit formulae in terms of $\theta$-functions. The case of general matrix operators was analyzed by Dubrovin [51] (in our account we essentially follow [51]). Our aims were as follows.

1. The generalization of the methods of the authors to more than twodimensional matrix operators meets certain difficulties. However, a number of physically interesting non-linear equations give rise to such operators; for example, for three-dimensional operators as was pointed out by Zakharov and Manakov in [27], the equation for the interaction of wave packets in nonlinear media (see Ch. $1, \S 4$ of the present survey).

2. The application to the theory of Abelian varieties according to the scheme of [43] (see Ch. 2, $\$ 4$, of this survey). Here we can describe explicitly the universal fibering of Jacobian varieties that are not necessarily hyperelliptic.

3. As Tyurin has indicated, an interesting topic is the applicability of our technique to the classical problem of unirationality not only of the space of the universal fibering of Jacobian varieties, but also of the base of this fibering, that is, the unirationality of the space of moduli of algebraic curves. We show that the algebraic curves $\Gamma$ (see below) obtained within the framework of our construction form a necessarily unirational family, but 
the codimension of this family in the space of moduli is always non-zero.

We consider an $n$-dimensional linear differential first order matrix operator $L=\frac{d}{d x}+U(x)$, where $U(x)=\left(u_{i}^{j}(x)\right)$ is an $n \times n$ matrix, periodically dependent on $x$ with period $T$, and with zero diagonal elements $u_{i}^{i} \equiv 0(i=1, \ldots, n)$. We pose the eigenvalue problem for $L$ :

$$
L \psi=E A \psi, \quad \psi(x+T, E)=e^{p(E)} \psi(x, E) .
$$

Here $E$ is a complex parameter and $A$ is a constant diagonal matrix $A=\left(a_{i} \delta_{i}^{j}\right), \Sigma a_{i}=0$. It is convenient to consider at once the family of such problems parametrized by the matrices $A$.

Let $\mathbf{A}$ be the $(n-1)$-dimensional space of complex diagonal $n \times n$ matrices of trace zero. In what follows we consider functions of $n-1$ variables, parametrized by the matrices $A$; to each matrix $A \in \mathrm{A}$ there corresponds a variable $x_{A}$, where $x_{A+B}=x_{A}+x_{B}, x_{\lambda A}=\lambda x_{A}$; the set of these $(n-1)$ variables is denoted by $X$. Let $V=V(X)$ be an $n \times n$ matrix depending on $X$. For each matrix $A \in \mathrm{A}$ we construct an operator $L_{A}$ depending on the parameter $E$ :

$$
L_{\mathbf{A}}=\frac{\partial}{\partial x_{\mathrm{A}}}+[A, V(X)]-E A .
$$

The matrix $V$ is called the potential of the operator $L_{A}$, which acts on functions of $x_{A}$ and depends parametrically on the remaining variables $x_{B}(B \in \mathrm{A})$. We require that for different $A \in \mathrm{A}$ all the operators $L_{A}$ commute among themselves:

$$
\left\{\begin{array}{l}
{\left[L_{A}, L_{B}\right]=0 \Leftrightarrow \frac{\partial Q_{B}}{\partial x_{A}}-\frac{\partial Q_{A}}{\partial x_{B}}=\left[Q_{A}, Q_{B}\right]} \\
Q_{A}=[A, V]-E A, \quad Q_{B}=[B, V]-E B
\end{array}\right.
$$

The commutativity condition (3.2.3) is equivalent to the following nonlinear equation for $V(X)$ :

$$
\left[A, \frac{\partial V}{\partial x_{B}}\right]-\left[B, \frac{\partial V}{\partial x_{A}}\right]=[[A, V],[B, V]] \text {. }
$$

The formulae (3.2.3) show that the equation (3.2.4) admits a commutation representation by $n \times n$ matrices that are polynomially (linearly) dependent on the parameter $E$, In the sense of Ch. $2, \S 2$ (see (2.2.20)). Varying the matrices $A$ and $B$, we obtain a system of $n-2$ equations for the matrix $V(X)$ depending on the $(n-1)$-fold argument, so that knowing the dependence of $V$ on one variable $x_{A}$, we can determine the dependence on any other $x_{B}(B \in \mathrm{A})$ by solving the Cauchy problem for (3.2.4). In what follows, we always assume that $V(X)$ is a solution of the system (3.2.4) where $A$ and $B$ range over all the diagonal matrices $A$.

We now construct a set of commuting dynamical systems on the variety of matrices $V(X)$ that are solutions of the system (3.2.4). It is easy to see 
that the equation $\frac{\partial}{\partial x_{A}} \lambda_{A}=\left[\lambda_{A}, Q_{A}\right]$ has a unique solution in the form of a formal series in $1 / E$, beginning with $A$

$$
\lambda_{A}=\lambda_{0, A}+\frac{\lambda_{1, A}}{E}+\frac{\lambda_{2, A}}{E^{2}}+\ldots ; \quad \lambda_{0, A}=A .
$$

If $V(X)$ is a solution of (3.2.4), then $\lambda_{A}$ satisfies the following equation:

$$
\frac{\partial}{\partial x_{B}} \lambda_{A}=\left[\lambda_{A}, Q_{B}\right] \text {. }
$$

The matrix elements of $\lambda_{k, A}$ are polynomials in the elements of $V, \partial V / \partial x_{A}, \partial^{2} V / \partial x_{A}^{2} \ldots$ with constant coefficients, depending on $A$. Suppose now that $A_{1}, \ldots, A_{N+1} \in \mathrm{A}$.

DEFINITION 1. The Nth equation of $K-d V$ type is

$$
\left[A, \dot{V}-\left(\lambda_{A_{1}, N+1}+\lambda_{A_{2}, N}+\cdots+\lambda_{A_{N+1}, 1}\right)\right]=0,
$$

where the matrices $\lambda_{A_{k}, N-k+2}$ are defined by the algorithm (3.2.5) and $V=V(X, t)$ is a solution of (3.2.4).

The equation (3.2.6) admits a commutation representation by $n \times n$ matrices polynomially dependent on $E$, in the sense of Ch. $2, \S 2$ :

$$
\frac{\partial \Lambda}{\partial x_{A}}-\frac{\partial Q_{A}}{\partial t}=\left[\Lambda, Q_{A}\right]
$$

Here

$$
\Lambda=\Lambda_{A_{1}, N}(E)+\ldots+\Lambda_{A_{N+1}, 0}(E)
$$

where

$$
\text { (3.2.7") } \quad \Lambda_{A, K}=A E^{k}+\lambda_{1, A} E^{k-1}+\ldots+\lambda_{k, A} \quad(A \in \mathbf{A}) .
$$

It is easy to see that $\Lambda_{1, A}=-Q_{A}$, therefore (3.2.4) is the first equation of $\mathrm{K}-\mathrm{dV}$ type in the sense of Definition 1.

Let $V=V(X)$ be a periodic function of the variable $x_{A}$ with period $T$ (for simplicity we assume in the subsequent formulae that all the diagonal elements of $A$ are pairwise distinct). Then we have the eigenvalue problem for $L_{A}$ :

(3.2.8) $\quad L_{A} \psi\left(x_{A}, E\right)=0, \quad \psi\left(x_{A}+T, E\right)=e^{p(E)} \psi(x, E)$.

By analogy with Definition 1 in Ch. $2, \S 1$, we make the following definition:

DEFINITION 2. The potential $V$ is said to be finite-zoned for the operator $L_{A}$ if the eigenfunctions of the problem (3.2.8) are meromorphic on a Riemann surface $\Gamma$ of finite genus, which gives an $n$-fold covering of the $E$-plane. The surface $\Gamma$ is then called the spectrum of $L_{A}$.

If $V$ is almost-periodic as a function of $x_{A}$, then Definition 2 is modified as follows:

DEFINITION $2^{\prime}$. The potential $V$ is said to be finite-zoned for the operator $L_{A}$ if $L_{A}$ for all $E$ has an eigenfunction $\psi\left(x_{A}, E\right)$ that is meromorphic on a Riemann surface $\Gamma$ of finite genus that gives an $n$-fold cover- 
ing of the $E$-plane, with the "boundary condition" of (3.2.8) replaced by the following: the group of periods of the logarithmic derivatives of the coordinates of $\psi\left(x_{A}, E\right)$ is the same as the group of periods of $V$. The surface $\Gamma$ is called the spectrum of $L_{A}$.

We consider the stationary solutions of (3.2.6) (that is, those not depending on the time $t$ ). (We recall that $V(X)$ is a solution of the system (3.2.4).) To find them, as in $\mathrm{Ch} .2, \S 2$, we have a commutation representation of Lax type on matrices polynomially dependent on $E$ :

$$
\frac{\partial \Lambda}{\partial x_{A}}=\left[\Lambda, Q_{A}\right]
$$

where the matrix $\Lambda$ is defined by (3.2.7). We consider the Riemann surface $\Gamma$ of the algebraic function $W=W(E)$, where $W(E)$ is given by the equation

$$
R(W, E)=\operatorname{det}|W \cdot 1-\Lambda|=0 .
$$

Actually, $\Gamma$ is a complex algebraic curve in two-dimensional complex space with the coordinates $W$ and $E$, defined by (3.2.10). Since for an arbitrary $E$ there are $n$ values of $W(E), \Gamma$ gives an $n$-fold covering of the $E$-plane. The infinitely distant part of $\Gamma$ consists of $n$ ordered points $\{1\}, \ldots,\{n\}$; the order is determined by the conditions $W(E) \sim a_{1 i} E^{N}(E \rightarrow \infty)$ in a neighbourhood of $\{i\}$ (where $a_{1 i}$ is the $i$ th diagonal element of $A_{1}$ ).

Let $\pi$ be the factor group of the subgroup of scalar matrices in the group of all non-singular diagonal $n \times n$ matrices. Then $\pi$ acts on the potentials $V$ by the following rule:

$$
V \rightarrow \pi^{-1} V \pi .
$$

THEOREM 1. The stationary equation (3.2.9) is a totally integrable Hamiltonian system with $N \frac{n(n-1)}{2}$ degrees of freedom, and the coefficients of the polynomial $R(W, E)$ give a complete set of commuting polynomial integrals of (3.2.9).

2. The potential $V$ is finite-zoned for all the operators $L_{A}$; their spectrum is the Riemann surface $\Gamma$ defined by (3.2.10).

3. The set of finite-zoned potentials $V$ with a given spectrum $\Gamma$ is the space of the principal $\pi$-fibering over the Jacobian variety $\mathrm{J}(\Gamma)$ of $\Gamma$.

PROOF. As in Ch. $2, \S 2$, we see that $\Gamma$, defined by (3.2.10), does not depend on the variable $X$ and is invariant under all the dynamical systems of the form (3.2.6), that is, the coefficients of $R(W, E)$ are integrals of (3.2.9). Later we shall show that the systems (3.2.9) are Hamiltonian, hence the first part of the theorem follows by analogy with Ch. $2, \S 2$.

In the solution space of the equation $L_{A} f(x, E)=0$ we introduce the basis of solutions $c_{1}(x, y, E), \ldots, c_{n}(x, y, E)$ (denoting the variable $x_{A}$ simply by $x$ and regarding $A$ as fixed) such that $c_{i}^{j}(y, y, E)=\delta_{i}^{j}$ (where $y$ is a parameter). Let $\hat{T}(y, E)$ be the translation matrix for $L_{A}$ in the basis $c_{1}(x, y, E), \ldots, c_{n}(x, y, E)$ if $V$ is periodic in $x$. If $V$ evolves in 
time $t$ according to the K-dV type equation (3.2.6), then the time derivative for $\hat{T}$ has the form

$$
\frac{\partial}{\partial t} \hat{T}=[\hat{T}, \Lambda]
$$

with $\Lambda$ defined by $\left(3.2 .7^{\prime}\right)$. Since we look for stationary solutions of (3.2.6), the matrices $T$ and $\Lambda$ commute:

$$
\hat{T} \Lambda=\Lambda \hat{T} \text {. }
$$

Let $\psi(x, E)$ be an eigenfunction of the problem (3.2.8) $\left(x=x_{A}\right)$, normalized by fixing the value of one coordinate when $x=y$, for example, $\psi^{1}(y, E)=1$.

We note that

$$
\psi(x, E)=\sum_{j} \psi^{j}(y, E) c_{j}(x, y, E) .
$$

Here $\left(\psi^{1}(y, E), \ldots, \psi^{n}(y, E)\right)$ is an eigenvector of $\hat{T}(y, E)$, hence also of $\Lambda(y, E)$, by (3.2.11). Consequently, the coordinates $\psi^{j}(y, E)$ can be expressed rationally (with the normalization taken into account) in terms of the elements of the matrix $W(E) \cdot 1-\Lambda(y, E)$, that is, they are algebraic functions on the Riemann surface $\Gamma$ defined by (3.2.10). Therefore, by (3.2.12) $\psi(x, E)$ can be continued as a meromorphic function on the Riemann surface $\Gamma \backslash \infty$ (that is, away from the "infinitely distant part" of $\Gamma$ ). So we have proved that $L_{A}$ is finite-zoned with spectrum $\Gamma$. If $V$ is an almost-periodic solution of (3.2.9), then we define the eigenfunction $\psi$ by

$$
\psi(x, E)=\sum_{j} \xi^{j}(y, E) c_{j}(x, y, E),
$$

where $\left(\xi^{1}(y, E), \ldots, \xi^{n}(y, E)\right)$ is an eigenvector of $\Lambda(y, E)$ with the eigenvalue $W(E)$. The definition (3.2.12) is not contradictory because according to (3.2.9) $L_{A}$ commutes with the operator of multiplication by $\Lambda$. The finite-zone property is subsequently proved as in the periodic case (compare with the second method of proof of Corollary 2 in $\mathrm{Ch} .2, \S 2$ ).

We construct the matrix-valued function $\Psi(x, y, P)$, where $P$ is a point of $\Gamma$. Let $E$ be a point that is not a branch-point, so that for the given $E$ the problem (3.2.8) has exactly $n$ linearly independent arbitrarily ordered eigenfunctions $\psi_{1}(x, E), \ldots, \psi_{n}(x, E)$. We form from their coordinates a matrix $\psi_{i}^{j}(x, E)$. Let $\varphi_{i}^{j}(x, E)$ be its inverse, which exists because $\psi_{1}, \ldots, \psi_{n}$ are linearly independent. If $P \in \Gamma, P=(E, k)$, where $k$ labels the sheet, then we set

$$
\Psi_{i}^{j}(x, y, P)=\psi_{k}^{j}(x, E) \cdot \varphi_{i}^{k}(y, E) .
$$

This definition does not depend on the original ordering of the eigenfunctions $\psi_{1}, \ldots, \psi_{n}$ nor on their normalization. The function $\Psi(x, y, P)$ becomes meromorphic on the Riemann surface $\Gamma \backslash \infty$. 
We define the operation $\operatorname{Tr}_{P}$, which is important in what follows, of taking the trace of a function defined on $\Gamma$. Let $\varphi=\varphi(P)$ be such a function, $P \in \Gamma$, that is, $P$ is a pair $(E, k)$ where $k$ labels the sheet. If $E$ is not a branch-point, there are exactly $n$ points $(E, 1), \ldots,(E, n)$ on $\Gamma$ over $E$. We then set

$$
\text { (3.2.14) } \quad\left(\operatorname{Tr}_{P} \varphi\right)(E)=\varphi((E, 1))+\ldots+\varphi((E, n)) \text {. }
$$

Now $\operatorname{Tr}_{p} \varphi$ is a single-valued function on the $E$-plane. In particular, if

$$
G(x, y, E)=\left\{\begin{array}{cc}
\operatorname{Tr}_{P} \Psi(x, y, P) & (x \leqslant y), \\
0 & (x>y),
\end{array}\right.
$$

then $G(x, y, E)$ is the Green's matrix of $L_{A}$. Let $g(x, P)=\Psi(x, x, P)$.

Then $g(x, P)$ has the following important properties. $V$.

a) The group of periods of $g(x, P)$ is the same as that of the potential

b) It is algebraic on $\Gamma$.

c) It gives the "spectral decomposition" for the matrix $\Lambda(x, E)$ that is, $g^{2}=g, g(x,(E, k)) \cdot g(x,(E, l))=0$ for $k \neq l$ (where $k, l$ label the sheets), $\operatorname{Tr}_{P} g(x, P)=1, \operatorname{Tr}_{P} W \cdot g(x, P)=\Lambda(E, x)$.

d) $\frac{\partial g}{\partial x_{3}}=\left[g, Q_{B}\right]$ for any $B \in \mathbf{A}$.

e) The variational derivative of the functional $p(E)\{V\}$ defined in (3.2.8) (in the periodic case) has the form

$$
\frac{\delta p(E)}{\delta v_{i}^{j}(x)}=-\left(a_{i}-a_{j}\right) g_{i}^{j} \quad(\text { see }(2.1 .17)) \text {. }
$$

f) As $P \rightarrow\{k\}, g(x, P)$ has an expansion of the form

$$
g(x, P)=g_{0}+\frac{g_{1}}{E}+\frac{g_{2}}{E^{2}}+\ldots,
$$

where

$$
\begin{gathered}
g_{0_{i}}^{j}=\delta_{i}^{k} \cdot \delta_{k}^{j} ; \quad g_{1_{i}}^{j}=-\delta_{i}^{k} v_{i}^{j}+v_{i}^{j} \delta_{k}^{j} ; \\
g_{2_{i}}^{j}=\delta_{i}^{k}\left[\frac{v_{i}^{j^{\prime}}}{a_{i}-a_{j}}+\frac{1}{a_{i}-a_{j}} \sum_{s} v_{i}^{s}\left(a_{s}-a_{j}\right) v_{s}^{i}\right]+ \\
+\left[-\frac{v_{i}^{j^{\prime}}}{a_{i}-a_{j}}+\frac{1}{a_{i}-a_{j}} \sum_{s} v_{i}^{s}\left(a_{i}-a_{s}\right) v_{s}^{j}\right] \delta_{k}^{j}-v_{i}^{k} v_{k}^{j}+\delta_{i}^{k}\left(\sum_{s} v_{i}^{s} v_{s}^{j}\right) \delta_{k}^{j} .
\end{gathered}
$$

Here the dash denotes differentiation with respect to $x=x_{A}$.

From c) and e) it follows immediately that the systems (3.2.9) are Hamiltonian. Let us find the zeros and poles of the matrix elements $g_{i}^{j}(x, P)$, which are algebraic on $\Gamma$. From c) it follows that the poles of $g(x, P)$ are precisely the branch-points of $\Gamma$, that is, the points at which the different branches of the algebraic function $W(E)$ merge. We denote the set of branch-points by the symbol $\mathscr{D}_{w}$. There are $N n(n-1)$ of them. From the expansion $f$ ) it follows that at the infinitely distant part of $\Gamma$ 
all the $g_{i}^{j}$ have zeros and their disposition is as follows: for $i \neq j, g_{i}^{j}(x, P)$ has double zeros at all the points $P=\{k\}(k \neq i, j)$ and simple zeros at the points $P=\{i\}, P=\{j\} ; g_{i}^{i}(x, P)$ has double zeros at all the points $P=\{k\},(k \neq i)$, and $g_{i}^{i}(x, P)=1$ at $P=\{i\}$. There is a convenient notation to describe the distribution of zeros and poles of a function defined on $\Gamma$. Let $\varphi(P)$ be a function on $\Gamma$ having zeros of multiplicity $n_{1}$ at $P_{1}, n_{2}$ at $P_{2}, \ldots$, and poles of multiplicity $m_{1}$ at $Q_{1}, m_{2}$ at $Q_{2}, \ldots$... We express this by saying that $\varphi(P)$ has the divisor $D=n_{1} P_{1}+n_{2} P_{2}+\ldots-m_{1} Q_{1}-m_{2} Q_{2}-\ldots$ on $\Gamma$. It is clear that the whole divisor $D$ of $\varphi(P)$, that is, the set of zeros and poles of $\varphi(P)$ with their multiplicities taken into account, can be decomposed into the difference $D=D_{+}-D_{-}$, where $D_{+}=n_{1} P_{1}+n_{2} P_{2}+\ldots$ is the set of zeros (the divisor of zeros), and $D_{-}=m_{1} Q_{1}+m_{2} Q_{2}+\ldots$ the set of poles of $\varphi(P)$ (the divisor of poles). The degree of the divisor $D$ is defined to be

$$
\operatorname{deg} D=\sum_{i} n_{i}-\sum_{j} m_{j}=\operatorname{deg} D_{+}-\operatorname{deg} D_{-} .
$$

If $\varphi(P)$ is an algebraic function on $\Gamma$, that is meromorphic everywhere on $\Gamma$, then $\operatorname{deg} D=0$ (the number of zeros, counting multiplicities, is equal to the number of poles, counting multiplicities). In particular, the result on the zeros and poles of $g_{i}^{j}(x, P)$ on $\Gamma$ can be expressed concisely as:

(3.2.15) divisor $\left(g_{i}^{j}(x, P)\right)=\sum_{k \neq i, j} 2\{k\}+\{i\}+\{j\}-\mathscr{D}_{w}+\ldots=$

$$
=2 \Sigma-\{i\}-\{j\}-\mathscr{D}_{w}+\ldots,
$$

where $\Sigma=\sum_{k}\{k\}$, and the dots denote the unknown divisor of the zeros of $g_{i}^{j}(x, P)$ in the finite part of $\Gamma$. To find this we turn to a study of the analytic properties of the matrix $\Psi(x, y, P)$ defined by (3.2.13). We note the formula $\Psi(x, y, P)=c(x, y, E(P)) g(y, P)$ similar to (3.2.12). Therefore, the poles of the matrix elements $\Psi(x, y, P)$ lie only at the branch-points (that is, the divisor of poles of $\Psi_{i}^{j}(x, y, P)$ is equal to $\mathscr{D}_{w}$ for all $i$ and $j$ ). The matrix $\Psi(x, y, P)$ is of rank 1 ; its columns are eigenfunctions of $L_{A}$, which acts on the variable $x$, and differ only in normalization, and the rows are eigenfunctions of the adjoint operator $L_{\boldsymbol{A}}^{*}$, which acts on $y$ and is defined as follows:

$$
L_{A}^{*}=\frac{\partial}{\partial y}-Q_{A}^{T}(T \text { denotes the transpose }) .
$$

Therefore the relations

and

$$
\frac{\Psi_{i}^{k}(x, y, P)}{\Psi_{j}^{k}(x, y, P)}=\frac{g_{i}^{k}(y, P)}{g_{j}^{k}(y, P)}
$$

$$
\frac{\Psi_{k}^{i}(x, y, P)}{\Psi_{k}^{j}(x, y, P)}=\frac{g_{k}^{j}(x, P)}{g_{k}^{j}(x, P)} \text { do not depend on } k \text {. }
$$


From (3.2.16) and (3.2.16) it follows that the zeros of $\Psi_{i}^{j}(x, y, P)$ split into two parts: zeros depending on $j$ and on $x$, and zeros depending on $i$ and on $y$. We express this in the following way:

$$
\text { divisor of the zeros of }\left(\Psi_{i}^{j}(x, y, P)\right)=d_{i}(y)+d^{j}(x),
$$

where the divisors $d_{i}(x)$ and $d^{j}(y)$ have the form

$$
d_{i}(y)=P_{1 i}(y)+\ldots, \quad d^{j}(x)=Q_{1}^{j}(x)+\ldots
$$

Thus, we define the divisor of $g_{i}^{j}(x, P)$ as

(3.2.18) divisor $\left(g_{i}^{j}(x, P)\right)=2 \Sigma-\{i\}-\{j\}-\mathscr{D}_{w}+d_{i}(x)+d^{j}(x)$.

The function $g_{i}^{j}(x, P)$ is algebraic on $\Gamma$, hence the degree of its divisor is zero (the number of zeros is equal to the number of poles). Therefore, $\operatorname{deg}\left[d_{i}(x)+d^{j}(x)\right]=2\left[N \frac{n(n-1)}{2}-(n-1)\right]$. Since the operators $L_{A}$ and $L_{A}^{*}$ are entirely of equal standing, we have the important relation

(3.2.19) $\operatorname{deg} d_{i}(x)=\operatorname{deg} d^{j}(x)=N \frac{n(n-1)}{2}-(n-1)=\operatorname{genus}(\boldsymbol{\Gamma}) \equiv p$.

Hence $d_{i}(x)=P_{1 i}(x)+\ldots+P_{p i}(x), d^{j}(x)=Q_{1}^{j}(x)+\ldots+Q_{p}^{j}(x)$. The divisors $d_{i}(x)$ and $d^{j}(x)$ can therefore be regarded as points of the $p$ th symmetric power $S^{p} \Gamma$ of $\Gamma$ (see the definition in Ch. 2 , §3). We recall that the Abelian map $\mathfrak{A}$ from the $k$ th symmetric power of $\Gamma$ into its Jacobian variety

$$
\mathfrak{A}: \mathbf{S}^{k} \boldsymbol{\Gamma} \rightarrow \mathbf{J}(\boldsymbol{\Gamma}),
$$

is almost everywhere one-to-one if $k=p=$ genus $(\Gamma$ ). (More accurately, it is a birational equivalence, see (2.3.25).) The map $\mathfrak{U}$ has the following property: if $D=D_{+}-D_{-}$is the divisor of the zeros and of the poles of $\varphi(P)$, algebraic on $\Gamma$ then (by the classical theorem of Abel)

$$
\mathfrak{A}\left(D_{+}\right)=\mathfrak{A}\left(D_{-}\right) \text {. }
$$

We know that $g_{i}^{j}(x, P)$ is algebraic on $\Gamma$ and has a divisor of the form (3.2.8). Now, bearing (3.2.21) in mind we obtain a system of linear equations on $\mathbf{J}(\Gamma)$ for the quantities $\mathfrak{A}\left(d_{i}(x)\right), \mathfrak{A}\left(d^{j}(x)\right)$ :

(3.2.22) $\quad \mathfrak{A}\left(d_{i}(x)\right)+\mathfrak{A}\left(d^{j}(x)\right)=\mathfrak{A}\left(\mathscr{D}_{w}\right)-\mathfrak{X}(2 \mathbb{\Sigma}-\{i\}-\{j\})$.

It is easy to see that the specification of any one of the unknowns, for example, of $\mathfrak{A}\left(d^{1}(x)\right)$, determines all the remaining $\mathfrak{A}\left(d_{i}(x)\right), \mathfrak{A}\left(d^{j}(x)\right)$. Thus, we have constructed a correspondence

$$
V \rightarrow \eta, \quad \eta \in \mathbf{J}(\Gamma)
$$

where $V$ is a solution of (3.2.9) and the specification of $\eta \in \mathbf{J}(\Gamma)$ uniquely determines the distribution of the zeros of $g(x, P)$ (for fixed $x$ ) on $\Gamma$.

We now show that, as $x$ varies, the point $\eta$ on $\mathbf{J}(\Gamma)$ moves in a straight line, that is, 


$$
\eta(x)=\eta(y)+(x-y) \vec{U} ;
$$

where $\vec{U}$ is a constant vector. We consider the function $\widetilde{\Psi}(x, y, P)$ where

$$
\widetilde{\Psi}^{j}(x, y, P)=\Psi_{i}^{j}(x, y, P) / g_{i}^{j}(y, P)
$$

does not depend on $i$. Then $\widetilde{\Psi}^{j}(x, y, P)$ has on $\Gamma \backslash \infty$, zeros at the points of $d^{j}(x)$ and poles at the points of $d^{j}(y)$, and as $P \rightarrow\{k\}$, it has the asymptotic form $\exp \left\{\alpha_{k} E(x-y)\right\}$. The latter assertion follows from the formula

where

$$
\widetilde{\Psi}^{j}(x, y, P)=\exp \left\{\int_{y}^{x} \chi^{j}(\xi, P) d \xi\right\}
$$

$$
\chi^{j}(\xi, P)=a_{j} E-\sum_{s} u_{s}^{j}(\xi) \frac{g_{i}^{s}(\xi, P)}{g_{i}^{j}(\xi, P)},
$$

and from the expansions for $g_{i}^{j}(\xi, P)$ given in $\left.f\right)$. Hence we find ourselves in a situation similar to that of Ch. $2, \S 3$, in the proof of (2.3.30) and $\left(2.3 .30^{\prime}\right)$. Reasoning in exactly the same way, we obtain the relation on $\mathbf{J}(\Gamma)$

$$
\mathfrak{A}\left(d^{j}(x)\right)-\mathfrak{A}\left(d^{j}(y)\right)=(x-y) \vec{U},
$$

which is equivalent to (3.2.4). We shall choose the vector $\vec{U}$ below.

We have seen that the specification of $\eta$ on $\mathbf{J}(\Gamma)$ determines the position of the zeros of all the functions $\Psi_{i}^{j}(x, y, P)$ for arbitrary $x$ and $y$. It is easy to see that the "phases" $\chi^{j}(x, P)$ are likewise determined for all $\xi$. It remains to define the "amplitudes" of the functions $\Psi_{i}^{j}(x, y, P)$, that is, the functions $g_{i}^{j}(y, P)$. We note that $g_{i}^{i}(y, P)$ is uniquely determined by its zeros, and the functions $g_{i}^{j}(y, P), i \neq j$, are uniquely determined to within a constant factor: that is, for a given point $\eta \in \mathbf{J}(\Gamma)$ we can construct a matrix $\widetilde{g}(y, P)$, which is, in general, distinct from our $g(y, P)$ :

$$
\tilde{g}_{i}^{i}(y, P)=\varepsilon_{i}^{j} g_{i}^{j}(y, P),
$$

where $\varepsilon_{i}^{j}=$ constant. If we require that $\widetilde{g}^{2}=\tilde{g}$, then we find that $\varepsilon_{i}^{\tilde{j}}=\varepsilon_{i} / \varepsilon_{j}, \varepsilon_{1}, \ldots, \varepsilon_{n} \neq 0$, that is, the non-uniqueness in the definition of the "amplitudes" $g_{i}^{j}(y, P)$ of the functions $\Psi_{i}(x, y, P)$ lies in the action of the group $\pi$. The same non-uniqueness occurs also in the definition of the potential $V$. It is noteworthy that the action of $\pi$ on the variety of potentials $V$ commutes with all the dynamical systems of the form (3.2.6).

We give an explicit construction of $V$. We define the following functions on $\mathbf{J}(\Gamma)$ : let $\eta \in \mathbf{J}(\Gamma)$. We construct for $\eta$ the set of divisors $d_{i}$, $d^{j}$ of degree $p$ on $\Gamma$ such that $\mathfrak{U}\left(d^{1}\right)=\eta$, and the remaining $\mathfrak{A}\left(d_{i}\right)$, 
$\mathfrak{A}\left(d_{j}\right)$ are determined by the system of linear equations (3.2.22). Let $h_{i}^{j}(P)$ be an algebraic function on $\Gamma$ whose divisor of zeros and poles has the form

$$
2 \Sigma-\{i\}-\{j\}+d_{i}+d^{j}-\mathscr{D}_{w}
$$

(compare with (3.2.18)). We normalize $h_{i}^{j}$ as follows: for $i \neq j$ we require $h_{i}^{j}(P) \sim-\frac{1}{E}$, as $P \rightarrow\{i\}$; for $i=j$ we require that $h_{i}^{j}(\{i\})=1$. Let $\sigma_{i}^{j k}(\eta)$ be the coefficient of $\frac{1}{E^{2}}$ in the expansion of $h_{i}^{j}(P)$, as $P \rightarrow\{k\}$. Also, let $\rho_{i}^{k}(\eta)$ be the coefficient of $1 \backslash E^{2}$ in the expansion of $h_{i}^{i}(P)$, as $P \rightarrow\{k\}$. Then

$$
v_{i}^{j}(x)=v_{i}^{j}\left(x_{3}\right) \exp \left\{\int_{x_{0}}^{x} \sum a_{k} \sigma_{i}^{j k} d x\right\}
$$

where the integration is in the direction of $\vec{U}$, and the constants $v_{i}^{j}\left(x_{0}\right)$ satisfy the relations

$$
\left\{\begin{array}{l}
\frac{v_{i}^{k}\left(x_{0}\right) v_{k}^{j}\left(x_{0}\right)}{v_{i}^{j}\left(x_{0}\right)}=-\sigma_{i}^{j k}(\eta) \text { when } i \neq j, \\
v_{i}^{k}\left(x_{0}\right) v_{k}^{j}\left(x_{0}\right)=-\rho_{i}^{k}(\eta) .
\end{array}\right.
$$

As independent variables we can take, for example, the parameters $v_{i}^{1}\left(x_{0}\right), i=2, \ldots, n$.

We note now that the above arguments carry over trivially to the calculation of the time dependence on the variety of potentials $V$ that are solutions of the Hamiltonian system (3.2.9). We need merely replace the operators $L_{A}$ everywhere by $\frac{\partial}{\partial \tau}+\widetilde{\Lambda}$, where $\widetilde{\Lambda}$ is another matrix of the form (3.2.7) and the definition of $g$ is independent of the operator in question ( $g$ gives a "spectral decomposition" for $\Lambda$ ). We write down the law of time evolution of a point $\eta$ on $\mathbf{J}(\Gamma)$ for standard equations of $\mathrm{K}-\mathrm{dV}$ type

$$
\left[A, \dot{V}-\lambda_{N+1, B}\right]=0
$$

(the remaining ones are linear combinations of these). Then

$$
\eta(\tau)-\eta\left(\tau_{0}\right)=\vec{W} \cdot\left(\tau-\tau_{0}\right)
$$

The vector $\vec{W}$ can be found as follows. Let $\omega_{N, i}$ be an Abelian differential of the second kind with an $(N+1)$-fold pole at $P=\{i\}$, normalized by the conditions

$$
\oint_{\alpha_{j}} \omega_{N, i}=0, \quad(j=1, \ldots, p) .
$$

Here $\alpha_{i}, \beta_{i}(i=1, \ldots, p)$ is the set of cycles on $\Gamma$ with the intersection matrix of the form $\left(2.3 \cdot 21^{\prime}\right)$. 
Let

$$
\oint_{\beta_{j}} \omega_{N, i}=U_{j i} .
$$

Then the vector $\vec{W}$ in $(3.2 .30)$ has the form

$$
W_{j}=\sum_{i} b_{i} U_{j i}
$$

In particular, we have shown that the potential $V$ is finite-zoned for all the operators $\frac{\partial}{\partial \tau}+\widetilde{\Lambda}$, where $\widetilde{\Lambda}$ is of the form (3.2.7). This completes the proof of the theorem.

EXAMPLE. Let $n=2$. $V=\left(\begin{array}{ll}0 & v_{+} \\ v_{-} & 0\end{array}\right), A=\left(\begin{array}{rr}1 & 0 \\ 0 & -1\end{array}\right)$; we consider the $(N+1)$ th equation of $\mathrm{K}-\mathrm{dV}$ type. The matrix $\Lambda_{N+1}(E)$ has the form

$$
\begin{gathered}
\Lambda_{N+1}=\left(\begin{array}{cc}
Q & P_{+} \\
P_{-} & -Q
\end{array}\right), \quad \operatorname{deg} Q=N+1, \quad \operatorname{deg} P_{ \pm}=N, \\
P_{ \pm}=\mp 2 v_{ \pm} \prod_{j=1}^{N}\left(E-\gamma_{j}^{ \pm}\right), \quad Q=E^{N+1}+\ldots
\end{gathered}
$$

The Riemann surface $\Gamma$ has the form $W^{2}-R(E)=0$, where $R(E)=Q^{2}+P_{+} P_{-}$, deg $R=2 N+2$. The projection $g$ has the form

$$
g=\left(\begin{array}{cc}
\frac{Q+\sqrt{R}}{2 \sqrt{R}} & \frac{P_{+}}{2 \sqrt{R}} \\
\frac{P_{-}}{2 \sqrt{R}} & \frac{-Q+\sqrt{R}}{2 \sqrt{R}}
\end{array}\right) .
$$

The divisor $d_{2}+d^{1}$ is the complete inverse image of the points $E=\gamma_{1}^{+}, \ldots, E=\gamma_{N}^{+}$on $\Gamma$. Thus, $d^{1}$ comprises the $N$ points $P_{1}, \ldots, P_{N}$ lying over the points $E=\gamma_{1}^{+}, \ldots, E=\gamma_{N}^{+}$. Let $\Sigma E_{i}=0$, where $R(E)=\prod_{i=1}^{2 N+2}\left(E-E_{i}\right)$. Then

$$
\left\{\begin{array}{l}
v_{+}(x)=c \exp \left\{-2 \int_{x_{0}}^{x} \sum \gamma_{i}^{+} d x\right\}, \\
v_{-}(x)=\frac{\rho(x)}{v_{+}(x)} .
\end{array}\right.
$$

The algebraic function $\rho=v_{+} v_{-}$has the form

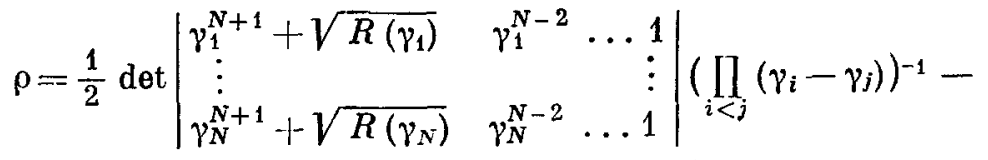

$$
\begin{aligned}
& -\frac{1}{4} \sum_{i<j} E_{i} E_{j} \quad\left(\gamma_{i}=\gamma_{i}^{+}\right) .
\end{aligned}
$$

We now write down the $\pi$-fibering over $\mathbf{J}(\Gamma)$ referred to in the theorem. To do this we rewrite $(3.2 .33)$ in the form 


$$
\left\{\begin{array}{l}
v_{+}=v_{+}\left(\eta, \eta_{0}, c\right)=c \exp \left\{\int_{\eta_{0}}^{\eta} \omega\right\}, \\
v_{-}=v_{-}\left(\eta, \eta_{0}, c\right)=\frac{\rho(\eta)}{v_{+}\left(\eta, \eta_{0}, c\right)} .
\end{array}\right.
$$

Here $c$ is the coordinate in the fibre of $\pi$ (for $n=2, \pi$ is one-dimensional), $\eta, \eta_{0} \in \mathbf{J}(\Gamma)$, and $c$ together with the initial point $\eta_{0}$ determine the potential $V$ in accordance with the theorem, and the dependence of $V$ on $\eta$ includes the dependence of the potential on a displacement along the trajectories of any one of the dynamical systems of the form (3.2.6); also,

$$
\omega=d \ln v_{+}=\frac{v_{+}^{\prime}}{v_{+}} d x+\sum_{i} \frac{\partial v_{+} / \partial t_{i}}{v_{+}} d t_{i}
$$

is a closed meromorphic differential on $\mathbf{J}(\Gamma)$. The periods of $\omega$ on the torus are given by the transition functions of the required $\pi$-fibering:

$c \rightarrow c \exp \oint \omega$ for a circuit of $\eta_{0}$ along a cycle in $\mathbf{J}(\Gamma)$. Here $\omega$ has the form

$$
\omega=-2\left(\sum \gamma_{i}\right) d x+\left(4 \sum_{i<j} \gamma_{i} \gamma_{j}-2 \sum E_{i} E_{j}\right) d t_{i}+\ldots
$$

In concluding this section we mention that, by analogy with $\mathrm{Ch} .2, \S 3$, we can prove a converse theorem: any finite-zoned potential (in the sense of Definitions 2 or $2^{\prime}$ ) of $L_{A}$ is a solution of a stationary equation of $\mathrm{K}-\mathrm{dV}$ type (that is, of the form (3.2.9)). The proof is based on the properties of $g(x, P)$ described above.

Simultaneously with [51] and [52], the papers [60] and [61] were completed, in which matrix operators are studied by the methods of Marchenko [44], [45].

\section{APPENDIX 1}

\section{NON-REFLECTIVE POTENTIALS AGAINST THE BACKGROUND OF FINITE- ZONE POTENTIALS. THEIR ALGEBRAIC-GEOMETRIC AXIOMATICS}

As was shown in $\mathrm{Ch} .2, \S 2$, every periodic or almost-periodic stationary solution of any higher order $\mathrm{K}-\mathrm{dV}$ equation is a finite-zone potential. However, these equations also have degenerate "separatrix" solutions. In [38] it was shown that rapidly decreasing separatrix solutions are nonreflecting potentials $u(x)$, associated with the so-called "multisoliton" solutions of the $\mathrm{K}-\mathrm{dV}$ equation, which have been studied in detail, for example in [20]. The general separatrix solutions of any of the higher order $\mathrm{K}-\mathrm{dV}$ equations are, as shown in [38], degenerate limits of conditionally periodic potentials, when some of the group of periods $T_{1}, \ldots, T_{n}$ tend to $\infty$. 
By virtue of the $\mathrm{K}-\mathrm{dV}$ equation it is natural to call the dynamics of such potentials "multisoliton solutions against a finite-zone background". A study of the inverse scattering problem for potentials of this kind was made by Krichever [49]; some months earlier for multisoliton potentials against the background of one-zone potentials the problem was solved by another method in [48]. We give here an account of the main arguments of [49], including the algebraic-geometric axiomatics of the class of non-reflective potentials against a finite-zone background. We begin by explaining the axiomatics. For convenience we recall some elementary concepts of algebraic geometry. By a divisor we mean a set of points $P_{i}$ on a Riemann surface $\Gamma$ of finite genus with multiplicities $k_{i}$, formally written as a sum:

$$
\text { divisor } D=\sum_{i} k_{i} P_{i} \text {. }
$$

By the divisor $(f)$ of a function we mean the divisor of its zeros and poles, where the multiplicities of the zeros are positive and those of the poles are negative. The divisor $(-D)$ has the form $\Sigma-k_{i} P_{i}$.

Formal addition of divisors is defined in the obvious way. A divisor whose multiplicities are all positive, $k_{i} \geqslant 0$, is said to be effective. We say that an effective divisor is greater than zero: $D \geqslant 0$.

DEFINITION. An algebraic function $f$ on $\Gamma$ is said to belong to the space $L(D)$ if $(f)+D \geqslant 0$. We define the degree of the divisor $D$, denoted by $n(D)$, to be the sum of the multiplicities of its points. We have the standard (Riemann) estimate

$$
\operatorname{dim} L(D) \geqslant n(D)-g+1 \quad(g=\text { genus } \Gamma),
$$

and equality holds for divisors of degree $n(D)>2 g-2$. Now let $\Gamma^{\prime}$ be a Riemann surface, doubly covering $\Gamma$ with branch-points $P_{1}, \ldots, P_{n}$, forming the "branching divisor" $\sum_{j=1}^{m} P_{j}, \Gamma \stackrel{\pi}{\rightarrow} \Gamma$ '. We define an involution $T$ on $\Gamma$, which interchanges the sheets of the covering, with $T P_{J}=P_{j}$. For any divisor $D$ we define the "conjugate divisor" $D^{+}=T(D)$.

We also assume that an algebraic function $E(P)$ is given on $\Gamma^{\prime}$, with simple poles at $Q_{1}, \ldots, Q_{s}$, whose sum forms the "divisor of poles" $D_{\infty}=-\sum_{\alpha=1}^{s} Q_{\alpha}$, and that all the poles of $E$ lie at branch-points $Q_{\alpha}=P_{J_{\alpha}}$. We denote by $\widetilde{E}=\pi^{*} E$ the lifting of $E$ to $\Gamma$.

DEFINITION. We say that a potential $u(x)$ on the interval $[a, b]$ has correct algebraic-geometric properties if there exists an eigenfunction $\psi(x, P), x \in[a, b] \quad(P \in \Gamma)$ such that

A) it satisfies the Sturm-Liouville equation

$$
-\psi^{\prime \prime}(x, P)+u(x) \psi(x, P)=\widetilde{E}(P) \psi(x, P)
$$

B) it is meromorphic everywhere on $\Gamma$, except at the poles of $\widetilde{E}$ at the 
points $\pi^{-1}\left(D_{\infty}\right)$ and the poles of $\psi(x, P)$ do not depend on $x$;

C) near poles of $\widetilde{E}$ the following asymptotic form holds:

$$
\psi(x, P) \sim \operatorname{const} e^{i \sqrt{\widetilde{E}(P)}\left(x-x_{0}\right)}
$$

(that is, $\psi e^{-i \sqrt{\widetilde{E}(P)}\left(x-x_{0}\right)}$ is regular at the poles of $\widetilde{E}$ ). The properties A) and $C$ ) are natural requirements in the axiomatics for this class of potentials. As for B), it is a natural generalization of properties of the Bloch function. We recall that the Bloch function $\psi$ is meromorphic on a Riemann surface $\Gamma$, doubly covering a rational (trivial) surface, the $E$-plane, on which $E$ has a pole at infinity (by definition). The two-sheeted character of the covering is a natural requirement because the Sturm-Liouville equation is of the second order, and we have a basis of solutions when there are two sheets (see Ch. 2, $\S 1$, and Ch. 3, §2). Apart from $\psi$ we also consider the function $\psi^{+}=T^{*} \psi$ (the sheets are exchanged) and their Wronskian

$$
W\left(\psi, \psi^{+}\right)=\psi \psi^{+\prime}-\psi^{+} \psi^{\prime}
$$

which is constant when condition A) is satisfied (that is, does not depend on $x$ ).

We now come to a statement of the "scattering data" problem on the Riemann surface $\Gamma$ defining the potential $u(x)$.

EXAMPLE 1 . In the case of a rapidly decreasing non-reflecting potential (see Ch. $2, \S 1$ ) a complete set of scattering data on the rational surface $\Gamma$ with the parameter $k=\sqrt{E}$ was the collection of points $i x_{1}, \ldots i x_{s}$ (in the upper half-plane $\operatorname{Im} k>0$ for real $u(x)$, or on the upper sheet of the surface $k=\sqrt{E}$ for real $E$ ), and the set of numbers $c_{1}, \ldots, c_{s}$. The numbers $i x_{1}, \ldots, i x_{s}$ determine the position of the discrete levels $E_{j}=-x_{j}^{2}$.

The eigenfunctions $f(x, k)$ and $g(x, k)$ defined by the conditions

$$
\begin{array}{ll}
f_{ \pm}(x, k) \rightarrow e^{ \pm i k x} & (x \rightarrow+\infty), \\
g_{ \pm}(x, k) \rightarrow e^{ \pm i k x} & (x \rightarrow-\infty),
\end{array}
$$

do not satisfy the conditions A), B), C). By analogy with the Bloch function (see Ch. 2), we introduce a new function $\psi\left(x, x_{0}, k\right)$ proportional to $f(x, k)$ by a factor depending rationally on $k$, and such that $\psi\left(x, x_{0}, k\right) \equiv 1$ when $x=x_{0}$. Then $\psi\left(x, x_{0}, k\right)$ satisfies A), B), C). Any point $P$ on $\Gamma$ is determined by the parameter $k=\sqrt{E}$, and the function $E(P)$ has the form $E=k^{2}$. It is easy to verify that the zeros $P_{j}(x)$ of $\psi$ lie over points $\gamma_{j}(x)$ and the poles $P_{j}\left(x_{0}\right)$ over points $\gamma_{j}\left(x_{0}\right) \quad(j=1, \ldots, N)$ on $\Gamma$. The "divisor of the poles" has the form

$$
D=\sum_{j=1}^{N} P_{j}\left(x_{0}\right) .
$$

The Wronskian $W\left(\psi, \psi^{+}\right)$vanishes at the branch-point $k=0$ and at the points of the discrete spectrum 


$$
Q^{ \pm}= \pm i x_{1}, \ldots, Q_{n}^{ \pm}= \pm i x_{n}, \quad E_{\alpha}=-x_{\alpha}^{2} \quad(\alpha=1, \ldots, n) .
$$

Clearly, $n=N$. The set of points

$$
\left[P_{1}\left(x_{0}\right), \ldots, P_{N}\left(x_{0}\right),+i x_{1}, \ldots,+i \varkappa_{N}\right],
$$

where the $P_{j}$ lie over $\gamma_{j}$, uniquely determine the potential $u(x)$. Equations in $x$ for the quantities $\gamma_{j}(x)$ for rapidly decreasing non-reflecting potentials were first found by Shabat [11], in the language of "conditional eigenvalues". At the branch-points $k=0$ and at all the points $k_{j}=i \varkappa_{j}$ the following equations hold:

or

$$
\psi\left(x, x_{0}, i x_{j}\right) \equiv \psi^{+}\left(x, x_{0}, i x_{j}\right)
$$

$$
W\left(\psi, \psi^{+}\right)=0, \quad k=i \varkappa_{j} .
$$

We call the pair of divisors

$$
D=\sum_{j} P_{j}\left(x_{0}\right) \quad \text { and } \quad d=\sum_{j} Q_{j}^{+}
$$

a "complete set of scattering data".

EXAMPLE 2. Suppose that we are given a non-degenerate $N$-zone periodic or almost-periodic potential (real or complex) with the Bloch eigenfunction $\psi^{ \pm}\left(x, x_{0}, E\right)$, as defined in Ch. 2. The divisor of the poles has the form

$$
D=\sum_{j=1}^{N} P_{j}\left(x_{0}\right),
$$

where the $P_{j}\left(x_{0}\right)$ lie on $\Gamma$ over the points $\gamma_{j}\left(x_{0}\right)$ of the $E$-plane. The function $\psi$ satisfies the conditions A), B), C), and its Wronskian has the form

$$
W\left(\psi, \psi^{+}\right)=2 i \frac{\sqrt{R(E)}}{\prod_{j}\left(E-\gamma_{j}\left(x_{0}\right)\right)}, \quad R(E)=\prod_{i=1}^{2 N+1}\left(E-E_{i}\right) .
$$

The Wronskian vanishes only at the branch-points $E_{i}$, which are completely determined by $\Gamma$ and the involution $T$ interchanging the sheets. In this case the divisor of poles $D$ completely determines the potential $u(x)$, by the results of Ch. 2.

We now pass on to the general case of potentials with correct algebraicgeometric properties.

Let $\psi(x, P)$ be a function on $\Gamma$ satisfying B) and C), and let $D$ be its divisor of poles. Let $d=\Sigma \lambda_{i} x_{i}$ be another effective divisor (where the $\lambda_{i}$ are numbers and the $x_{i}$ are points on $\Gamma$ ).

DEFINITION. We say that the pair of effective divisors $(D, d)$ is compatible if the following conditions are satisfied:

$$
\begin{aligned}
& n(d)=\sum \lambda_{i}=\operatorname{dim} L_{-}(\Delta)-1, \\
& \operatorname{dim}\left[L(\Delta-d) \cap L_{-}(\Delta)\right]=1,
\end{aligned}
$$


where the effective divisor $\Delta$ has the form

$$
\Delta=D+D^{+}-D_{\infty}
$$

$D^{+}=T(D), D_{\infty}$ is the divisor of poles of the function $E$ on $\Gamma$, and $L_{-}(\Delta)$ is the subspace of rational functions $f$ on $\Gamma$ that change sign under the interchange $T$ of sheets and such that $(f)+\Delta \geqslant 0$.

Let us explain this definition. The Wronskian $W\left(\psi, \psi^{+}\right)$belongs to $L_{-}(\Delta)$; for it has poles of the first order at the points of $D_{\infty}$, it can have poles at the points of the divisors $D$ and $D^{+}$, by definition, and finally, it changes sign when the sheets $T$ are interchanged. The divisor $d$ arises from those zeros of $W\left(\psi, \psi^{+}\right)$that do not lie at branch-points of $\Gamma$. Now we have the following proposition.

PROPOSITION 1. If $\psi$ satisfies the requirements $\mathrm{B}$ ) and $\mathrm{C}$ ) above, and if the divisor $D$ of its poles and the divisor $d$ of the zeros of the Wronskian $W\left(\psi, \psi^{+}\right)$form a compatible pair $(D, d)$, then $\psi$ satisfies a Sturm-Liouville equation for a certain potential $u(x)$. Conversely, if $\psi$ satisfies the requirements $\mathrm{A}), \mathrm{B}), \mathrm{C})$ and if $D$ is the divisor of its poles, then there exists a $d$ such that the pair $(D, d)$ is compatible.

The proof of the direct assertion follows from the fact that the Wronskian $W\left(\psi, \psi^{+}\right)$belongs to the space $L(\Delta-d) \cap L_{-}(\Delta)$, which is onedimensional according to the compatibility condition for $(D, d)$.

Thus, as $x$ varies, the Wronskian as a function of $P$ is simply multiplied by a constant $c(x)$. However, it clearly follows from Condition $C$ ) that $c(x) \equiv 1$. Hence $d W / d x \equiv 0$ from which the direct assertion follows easily. The expression $\frac{\psi^{\prime \prime}}{\psi}+E$ does not depend on $E$, as follows from its properties. The proof of the converse assertion follows easily from the definition of a compatible pair. If the divisor $d$ has the form

$$
d=\sum_{i} \lambda_{i} x_{i}
$$

then at the points $x_{i}$ the following identities hold (identically in $x$ ):

$$
\begin{gathered}
\left(\psi \equiv \psi^{+}\right)_{x_{i}}, \ldots,\left(\frac{d^{q}}{d z^{q}} \psi \equiv \frac{d^{q}}{d z^{q}} \psi^{+}\right)_{\varkappa_{i}}, \ldots \\
\left(q=0, \ldots, \lambda_{i}-1\right),
\end{gathered}
$$

where $z$ is a local parameter on $\Gamma$ near $x_{i}$.

In [49] the following theorem is proved.

THEOREM. If the inverse scattering problem is soluble for any compatible pair of effective divisors $(D, d)$ (that is, if there exist $u(x)$ and $\psi(x, P)$ with the correct algebraic-geometric properties), then the Riemann surface $\Gamma^{\prime}$ is rational, the function $E$ on it has exactly one pole at $E=\infty$ and $\Gamma$ is hyperelliptic and covers the E-plane twice. (All the potentials $u(x)$ with correct algebraic-geometric properties in this case satisfy one of the higher order $K-d V$ equations.)

We do not prove this theorem here (it follows in a straightforward way 
from some very simple algebraic-geometric facts). The theorem completes the axiomatics of the class of potentials satisfying equations of K-dV type. In fact, all the potentials of this class can be defined as meromorphic almost-periodic functions on the whole complex $x$-plane with a group of $2 n$ periods $T_{1}, \ldots, T_{n}, T_{1}^{\prime}, \ldots, T_{n}^{\prime}$, where any part of the periods can degenerate and become infinite. The number of poles of $\psi(x, P)$ (the degree of the divisor $n(D)$ ) must not be smaller than the genus of $\Gamma$ :

$$
N=n(D) \geqslant g \text {, }
$$

a degree of $n(D)$ for this class of potentials is $N-g$. We now give an explicit construction of this class of potentials.

In the first place, we can use the equations of Ch. $2, \S 2$, for the poles $\gamma_{j}\left(x_{0}\right)$ in the variable $x_{0}$ (or the zeros $\gamma_{j}(x)$ ) of the Bloch function $\psi_{ \pm}\left(x, x_{0}, E\right)$, which can be defined naturally in the given case, for example, by means of the formula

where

$$
\psi_{ \pm}\left(x, x_{0}, E\right)=c\left(x, x_{0}, E\right)+i \chi\left(x_{0}, E\right) s\left(x, x_{0}, E\right),
$$

$$
\chi\left(x_{0}, E\right)=\frac{\varphi(E) \sqrt{R(E)}-\frac{i}{2} \frac{d}{d x_{0}} \prod_{j=1}^{N}\left(E-\gamma_{j}\left(x_{0}\right)\right)}{\prod_{j=1}^{N}\left(E-\gamma_{j}\left(x_{0}\right)\right)},
$$

$R(E)=\prod_{i=1}^{2 n+1}\left(E-E_{i}\right)$ is a polynomial defining $\Gamma$, and

$$
\varphi(E)=\prod_{j=1}^{q}\left(E-i x_{j}\right)^{\lambda_{j}}
$$

is the polynomial defined by $d$.

$$
d=\sum_{j=1}^{q} \lambda_{j} Q_{j}
$$

where the $Q_{j}$ lie above the points $i x_{j}$ on the $E$-plane. One can imagine that $R(E) \cdot[\varphi(E)]^{2}$ is obtained as a result of degeneration of a more complicated Riemann surface for which all the roots $i x_{j}$ are of multiplicity $2 \lambda_{j}$ (we may assume that $\left.\lambda_{j}=1\right)$. For the poles $\gamma_{j}\left(x_{0}\right)$ we obtain the equation similar to (2.3.12)

$$
\frac{d \gamma_{j}}{d x_{0}}=2 i \frac{\varphi\left(\gamma_{j}\right) \sqrt{R\left(\gamma_{j}\right)}}{\prod_{k \neq j}\left(\gamma_{j}-\gamma_{k}\right)} .
$$

An equation like (2.4.3) is obtained for the time dynamics under the K-dV equation and its higher order analogues by the formal change

$$
\sqrt{R\left(\gamma_{i}\right)} \mapsto \sqrt{R\left(\gamma_{j}\right)\left[\varphi\left(\gamma_{j}\right)\right]^{2}}
$$

For a Riemann surface of genus $g=0$ we can solve these equations in 
the obvious way and obtain rapidly decreasing non-reflecting potentials and multisoliton solutions for the K-dV equations (see [11], [8], [20]).

For a Riemann surface $\Gamma$ of genus $g=1$ the equations can be solved without difficulty in terms of elliptic functions. We do not perform this integration, because the resulting formulae are already published in [48], [49].

Of course, for all $g \geqslant 2$ all these equations can be solved in terms of hyperelliptic functions on the Riemann surface. We draw attention to the following circumstance: if $\Omega_{k}$ are differentials of the first kind on $\Gamma$, normalized as in $\mathrm{Ch} .2, \S 3$, then we have the Abel map $\mathscr{A}$ :

$$
\eta_{k}=\sum_{j=1}^{N} \int_{P_{j}\left(x_{0}\right)}^{P_{j}(x)} \Omega_{k} \quad(k=1, \ldots, n),
$$

, (where $n$ is the genus of $\Gamma$ and $N$ is the number of poles $P_{j}\left(x_{0}\right)$ and zeros $P_{j}(x)$ of $\left.\psi_{ \pm}\left(x, x_{0}, E\right)\right)$. As before, the parameters $\eta_{k}$ on the torus (Jacobian variety) $\mathbf{J}(\Gamma)$ are such that

$$
\frac{d \eta_{k}}{d x}=U_{k}=\text { const }, \quad \frac{d \eta_{k}}{d t_{m}}=W_{k}^{m}=\text { const }
$$

(by virtue of all higher $\mathrm{K}-\mathrm{dV}$ equations of order $m$ ). Moreover, the points of the divisor $d=\Sigma \lambda_{j} Q_{j}$ depend neither on $x$ nor on the time. However, there remain $(N-n)$ unknown parameters whose dynamics is not contained in $\mathbf{J}(\Gamma)$. The Abel map here has the form

$$
\mathbf{S}^{N}(\mathbf{\Gamma}) \stackrel{A}{\rightarrow} \mathbf{J}(\boldsymbol{\Gamma}),
$$

where the inverse image $A^{-1}\left(\eta_{1}, \ldots, \eta_{n}\right)$ of a point is the complex projective space $\mathrm{CP}^{N-n}$. In [49] the following results are obtained.

Let $N=n+k$ and let $P_{1}\left(x_{0}\right), \ldots, P_{N+k}\left(x_{0}\right)$ be the poles of $\psi(x, P), D=P_{1}+\ldots+P_{n+k}$ and let $d=x_{1}+\ldots+x_{k}$ be that half of of the zeros of the Wronskian $W\left(\psi, \psi^{+}\right)$that do not lie at branch-points of the surface

$$
y_{2}=\prod_{i=1}^{2 n+1}\left(E-E_{i}\right)
$$

and chosen on the upper sheet. According to the results above, the pair $(D, d)$ uniquely determines the potential $u(x)$ with the eigenfunction $\psi(x, P)$. Suppose that $u_{i}(x)$ are the $n$-zone potentials on $\Gamma$ given by the divisors of the poles

$$
\left[P_{1}\left(x_{0}\right), \ldots, P_{n-1}\left(x_{0}\right), P_{n+i}\left(x_{0}\right)\right] \leftrightarrow u_{i}(x),
$$

and $\psi_{i}(x, P)$ the corresponding Bloch eigenfunctions.

PROPOSITION 2. The eigenfunction $\psi$ for $u(x)$ can be represented in the form 


$$
\psi(x, P)=\sum_{i=1}^{k} a_{i}(x) \psi_{i}(x, P),
$$

where the $a_{i}(x)$ do not depend on the point of the Riemann surface (on E) and can be determined from the system of equations

$$
\left\{\begin{array}{c}
\sum_{i=1}^{k} a_{i}(x)\left[\psi_{i}\left(x, x_{s}\right)-\psi_{i}^{+}\left(x, x_{s}\right)\right]=0 \quad(s=1, \ldots, k), \\
\sum_{i=1}^{k} a_{i}(x)=1 .
\end{array}\right.
$$

Now let $K(x)=\int_{x_{0}}^{x} u(x) d x, K_{i}(x)=\int_{x_{0}}^{x} u_{i}(x) d x$.

PROPOSITION 3.

$$
K(x)=\sum_{i=1}^{k} a_{i}(x) K_{i}(x) .
$$

We also introduce the "monosoliton potentials against the background of $n$-zone potentials". We can show that the integral $K(x)$ of a multisoliton potential against the background of an $n$-zone potential can be expressed rationally in terms of the similar integrals $K_{i j}(x)$ of the "monosoliton potentials against the background of $n$-zone potentials" and of the $n$-zone potentials $K_{i}(x)$ themselves. This rational representation can be thought of as a non-linear analogue of the superposition of the monosoliton solutions against the background of $n$-zone solutions with a given Riemann surface

$\Gamma$. For real bounded potentials $u(x)$ associated with $\Gamma: y^{2}=\prod_{j=1}^{2 n+1}\left(E-E_{j}\right)$, where $-\infty<E_{1}<\ldots<E_{2 n+1}<\infty$, the points $x_{j}$ lie in the intervals $E_{2 k}<x_{s}<E_{2 k+1}\left(-\infty<x_{s}<E_{1}\right)$, and the poles $\gamma_{j}\left(x_{0}\right)$ lie one each in the intervals obtained (complementary to the solution zones and the points $x_{j}$.) Then $\psi_{i}^{+}\left(x, x_{s}\right) \rightarrow 0$ and $\psi\left(x, x_{s}\right) \rightarrow 0$, as $x \rightarrow \pm \infty$, as follows from Proposition 2.

COROLLARY. Under these hypotheses about the distribution of the poles $\gamma_{j}\left(x_{0}\right)$ and of the points $x_{j}$ the potential $u(x)$ is smooth and bounded in $x$, and as $x \rightarrow \pm \infty$, the potential tends exponentially to the finite-zone potential $u_{ \pm}(x)$, where $u_{+}(x)$ is given on $\Gamma$ by the divisor of the poles of the Bloch function, which is equivalent to $D-d$, while $u_{-}(x)$ is given by a divisor equivalent to $D-d^{+}$(we recall that any divisor of degree $n$ is equivalent to an effective divisor, that is, to a sum of $n$ distinct points; to say that two divisors $D_{1}$ and $D_{2}$ are equivalent means that the difference $D_{1}-D_{2}$ is the complete divisor of the zeros and poles $(f)$ of an algebraic function $f$ on $\Gamma$ ). 
For a surface of genus $n=1$ the difference of the two potentials $u_{ \pm}(x)$ reduces to a phase shift, as indicated in [48].

In the general case this "displacement" of the potential on the torus $\mathbf{J}(\Gamma)$ is given by the divisor $\left(d-d^{+}\right)$, as follows from the corollary; the corresponding displacement vector in the parameter $\eta_{k}$ on the Jacobian variety is

$$
\begin{gathered}
\eta_{q}^{-\infty,+\infty}=\sum_{j=1}^{k} \int_{x_{j}^{+}}^{x_{j}^{-}} \Omega_{q} \quad(q=1, \ldots, n), \\
d=x_{1}^{+}+\ldots+x_{k}^{+}, \quad d^{+}=x_{1}^{-}+\ldots+x_{\bar{k}}^{-},
\end{gathered}
$$

where the points $x_{j}^{ \pm}$lie over $x_{j}$ of the $E$-plane on the upper and lower sheets, respectively, and the integral is over "half" of a cycle $a_{s}$ joining the points $x_{j}^{ \pm}$, in the lacuna in which $x_{j}$ lies. Here the $\Omega_{q}$, normalized as in Ch. 2, §3, form a basis of differentials of the first kind.

For monosoliton potentials against the background of $n$-zone potentials with a single point $x_{1}=x$, we obtain from Proposition 3

$$
K=\frac{\varphi_{2}}{\varphi_{2}-\varphi_{1}} K_{1}(x)+\frac{\varphi_{1}}{\varphi_{1}-\varphi_{2}} K_{2}(x),
$$

where

$$
\begin{aligned}
\varphi_{i} & =\psi_{i}(x, x)-\psi_{i}^{+}(x, x), \\
K & =\int u d x, \quad K_{i}=\int u_{i} d x .
\end{aligned}
$$

For the eigenfunctions $\psi_{i}$ we can use a modification of Its' formula [54]:

$$
\text { (A.1.3) } \quad \psi_{i}(x, P)=e^{\left(x-x_{0}\right) \int \Omega} \frac{\theta\left(\eta(P)+\left(x-x_{0}\right) \vec{U}+\eta^{0 i}-\vec{K}\right)}{\theta\left(\eta(P)+\eta^{0 i}-\vec{K}\right)}
$$

(for the definition of the differentials here, see Ch. 2, §3), where

$$
\begin{gathered}
\eta_{j}(P)=\int_{\infty}^{P} \Omega_{j}, \\
\eta_{j}^{0 i}=\sum_{k=1}^{n-1} \int_{\infty}^{P_{k}\left(x_{0}\right)} \Omega_{j}+\int_{\infty}^{P_{n+i}\left(x_{0}\right)} \Omega_{j} .
\end{gathered}
$$

The time dynamics is included automatically. (A.1.3) remains true at all times $t$, and for the eigenfunctions $\psi_{i}(x, t, P)$, which depend on the time by virtue of the $\mathrm{K}-\mathrm{dV}$ equation, we have

(A.1.4)

$$
\psi_{i}(x, t, P)=e^{\left(x-x_{0}\right) \int \Omega+i\left(t-t_{0}\right) \int \omega_{2}} \frac{\theta\left(\eta(P)+\left(x-x_{0}\right) \vec{U}+\left(t-t_{0}\right) \vec{W}^{(1)}+\eta^{0 i}-\vec{K}\right)}{\theta\left(\eta(P)+\eta^{0 i}-\vec{K}\right)},
$$

in which the differential $\omega_{2}$ has zero a-periods and a singularity $d z / z^{4}$, as 
$E \rightarrow \infty$, where $z=1 / \sqrt{E}$.

\section{APPENDIX 2}

\section{ANOTHER METHOD OF OBTAINING SOME THEOREMS IN CH. 2, §2}

Quite recently (at the beginning of 1975) a preprint of a new paper by Lax has reached us, in which he essentially develops the results of his earlier paper [50], which was mentioned in the Introduction and was discussed in Ch. $2, \S 2$. Although the actual results are contained in the previously published paper [38], the proofs differ substantially from the methods of [38]. We quote the basic arguments from Lax's preprint, whose main results are as follows. ${ }^{1}$

1) A stronger form is given of the result of [50] on the spectrum of a Schrödinger operator with periodic potential $u(x)$ satisfying any one of the stationary higher order K-dV equations (2.2.22). Stated in our language, although the theorem in [50] that these potentials are finite-zoned, is nonconstructive it is now also proved that the number of forbidden zones does not exceed $n$ (true, this proof is also non-constructive, and in Lax's paper there is no analogue of the algorithm for finding the boundaries of the zones which is described in [38] and in Ch. $2, \S 2$ of this survey. This will be clear from the derivation below).

2) Polynomial integrals are found of the stationary K-dV equations (2.2.22) by another method than that of [38]. As stated already, the boundaries of the zones have so far not been expressed in terms of these integrals. $^{2}$

3 ) It is proved that the simple eigenvalues of the operator $L$ are commutative (or involutory), and also that all the integrals $p\left(E_{j}\right)$ are involutory, where $p(E)$ is the Bloch dispersion law. The fact that the discrete eigenvalues of a rapidly decreasing potential are involutory was already established in [21]; for the periodic case this was also well known after [21]; a rigorous proof was published, for example, in [66]. As for the function $p(E)$, it is known that it is determined by the spectrum of the periodic problem (including degenerate levels), since the trace of the translation matrix $a_{R}=\frac{1}{2} \mathrm{Sp} \hat{T}$ is an entire function of order $\frac{1}{2}$ and is completely determined by the levels of the periodic problem $a_{R}=1$ including their multiplicities. (In classical terminology, an eigenvalue $e^{i p(E)}$ of the translation matrix $\hat{T}$ is called a "Flock exponent".) Since for almost any small perturbation $u+\delta u$ the eigenvalues become non-degenerate and the functional $p(E)$ is

1. Lax's paper has now been published [63].

2 A construction of these integrals was also achieved simultaneously and independently in the survey [62] - see the "Concluding remarks" at the end of this paper. 
smooth, the vanishing of the Poisson brackets $\left[p(E), p\left(E^{\prime}\right)\right] \equiv 0$ follows formally from [66]. This was pointed out by Faddeev (see the Introduction and $[38], \S 1)$.

PROOF OF 1). We consider the higher order K-dV equation and its operator representation

$$
\dot{u}=\frac{\partial}{\partial x}\left(\sum c_{i} \frac{\delta I_{n-\hat{i}}}{\delta u(x)}\right), \quad \dot{L}=[A, L],
$$

where $A=\Sigma c_{i} A_{n-i}$. We know that $A$ is a skew-symmetric operator. If $\dot{u}=0$ and $\dot{L}=0$, then $[L, A]=0$. Hence, if $L \psi_{j}=\lambda_{j} \psi_{j}$ and $\lambda_{j}$ is a nondegenerate level of the periodic problem, then $A \psi_{j}=\mu_{j} \psi_{j}$. This follows from elementary algebra. From the skew symmetry it follows that $\mu_{j}$ is purely imaginary. From the reality of the operator and of $\psi_{j}$ it follows that $\mu_{j}$ is real. Therefore, $\mu_{j}=0$. Hence, all the non-degenerate periodic eigenfunctions $\psi_{j}$ of $L$ satisfy $A \psi_{j}=0$. Since the order of $A$ is $2 n+1$, we see that there can be no more than $2 n+1$ non-degenerate levels $\lambda_{j}$ for $L$, because all the $\psi_{j}$ must be linearly independent. Thus, 1) is proved. The proof is non-constructive: it is not clear so far how to determine the disposition of the levels $\lambda_{j}$ by this method.

PROOF OF 2). We use the result of [21], [22] that the integrals $I_{m}=\int_{0}^{T} P_{m}\left(u, u^{\prime}, \ldots, u^{(m)}\right) d x$ are involutory. The Poisson brackets has the form

$$
\left[I_{n}, I_{m}\right]=\int_{0}^{T}\left(\frac{\delta I_{m}}{\delta u(x)} \frac{d}{d x} \frac{\delta I_{n}}{\delta u(x)}\right) d x,
$$

and we know that $\left[I_{n}, I_{m}\right]=0$. From the vanishing of this integral for an arbitrary periodic function $u(x)$ it follows, clearly, that

$$
\frac{\delta I_{m}}{\delta u(x)} \frac{d}{d x} \frac{\delta I_{n}}{\delta u(x)}=\frac{d}{d x} J_{m n}\left(u, u^{\prime}, \ldots\right),
$$

where $J_{m n}$ is a polynomial in $u, u^{\prime}, u^{\prime \prime} \ldots$ Obviously,

$$
J_{m n}+J_{n m}=\frac{\delta I_{n}}{\delta u(x)} \frac{\delta I_{m}}{\delta u(x)} .
$$

We consider the stationary equation

$$
\sum_{i=0}^{n} c_{i} \frac{\delta I_{n-i}}{\delta u(x)}=d, \quad \text { or } \quad \sum_{i=0}^{n+1} c_{i} \frac{\delta I_{n-i}}{\delta u(x)}=0,
$$

where $d=c_{n+i}, I_{-1}=-\int_{0}^{T} u d x$. Multiplying this equation by $\frac{d}{d x} \frac{\delta I_{m}}{\delta u(x)}$ and using (A.2.2), we obtain 


$$
\frac{d}{d x}\left(\sum_{i=0}^{n+1} c_{i} J_{n-i, m}\right)=0 .
$$

Let

$$
\text { (A.2.4' } \quad \widetilde{I}_{m}=\sum_{i=0}^{n+1} c_{i} J_{n-i, m} \quad(m=0, \ldots, n-1) .
$$

By (A.2.4) all the polynomials $\widetilde{I}_{m}$ are integrals of (2.2.22), and 2) is proved. It is easy to prove that the integrals $I_{m}$ are algebraically independent. By a direct calculation it can be shown that the $\widetilde{I}_{m}$ are also conserved in time under the higher order $\mathrm{K}-\mathrm{dV}$ equations (this is done in the preprint).

Of course, from this it follows more or less that these integrals $\widetilde{I}_{m}$ can be expressed in terms of the boundaries of the zones of the potential. Therefore, by the results of $\mathrm{Ch} .2, \S 2$, they can be expressed in terms of the constants $c_{0}=1, c_{1}, \ldots, c_{n}, c_{n+1}$ and the integrals $J_{\alpha}$. However this expression is not clear so far, and Lax gives no indication on how to catculate the boundaries of the zones in terms of the integrals $\widetilde{I}_{m}$ and the constants $c_{0}, \ldots, c_{n+1}\left(c_{0}=1, c_{n+1}=d\right)$ (see [65]).

We note, finally, that it follows trivially from these results that the set of periodic potentials for a given spectrum is an $n$-dimensional real algebraic variety in $R^{2 n}$ whose bounded connected components are isomorphic to the tori $T^{n}$. The complexification of these varieties and almost-periodic potentials are not discussed in Lax's paper. In his exposition Lax starts out from the following problem: how does one minimize the functional $I_{n}$ under given constraints $\left\{I_{k}=a_{k}\right\}(k<n)$ over the class of periodic functions with a given period?

\section{APPENDIX 3}

\section{ON THE USE OF LINEAR AND NON-LINEAR TRACE FORMULAE FOR THE INTEGRATION OF EQUATIONS OF K-dV TYPE AND THE EXPRESSION OF THE BLOCH SOLUTION OF THE SCHRÖDINGER EQUATION IN TERMS OF A $\theta$-FUNCTION}

In the mathematical literature, beginning with the papers [13] and [14] of Gel'fand, Levitan, and Dikii which were completed in 1953-1955, there are many publications dealing with the derivation of various identities for sums of powers of the eigenvalues of linear differential operators, in other words, trace formulae.

Formulae of this kind which we call non-linear trace formulae, have already appeared in the main part of this survey. In fact, we have established the relation

$$
\sum_{i=1}^{n} \gamma_{i}(x, t)=\frac{-u(x, t)}{2}+\frac{1}{2} \sum_{j=1}^{2 n+1} E_{j},
$$


and from $\left(2.3 .11^{\prime}\right)$ it follows at once that

$$
\sum_{i=1}^{n} \gamma_{i}^{2}(x, t)=\frac{u_{x x}(x, t)}{4}-\frac{u^{2}(x, t)}{2}+\frac{1}{2} \sum_{j=1}^{2 n+1} E_{j} .
$$

The computations leading to (A.3.2) can easily be generalized to higher order trace formulae; in particular, for the sums of the cubes of the quantities $\gamma_{i}$ we easily obtain the expression

$$
\sum_{i=1}^{n} \gamma_{i}^{3}=-\frac{u^{3}}{2}+\frac{3}{4} u u_{x x}+\frac{15}{32} u_{x}^{2}-\frac{3}{32} u_{x x x x}+\frac{1}{2} \sum_{i=1}^{n} E_{i}^{3} .
$$

We note that, to within the evaluation of the constants $\frac{1}{2} \sum_{i=1}^{n} E_{i}^{k}$, these formulae follow directly from the results of Dikii [14], as was explained in [54]. They can be obtained even more simply (again to within the constant $\frac{1}{2} \sum_{i=1}^{n} E_{i}^{k}$ ) from the fact mentioned earlier in this survey, that the polynomial

$$
P=\prod_{i}\left(E-\gamma_{i}(x, t)\right)
$$

satisfies the differential equation

$$
-P_{x x x}+4 P_{x} u(x, t)+2 u_{x} P=4 E P_{x} .
$$

We emphasize that the derivation of the trace formulae in our situation is connected with the Riemann surface $\Gamma$ of the function $\sqrt{\prod_{i=1}^{2 n+1}\left(E-E_{i}\right)}$ and holds for arbitrary complex values of the $E_{i}$. A foundation for trace formulae for arbitrary boundaries of zones can also be obtained using the following theorem of Its [54], which is of independent interest.

THEOREM. The function

in which

$$
\psi(x, t, E)=e^{i \omega(E) x} \frac{\theta(A(E)+x \vec{U}+t \vec{W}+l(0,0)) \theta(t \vec{W}+l(0,0))}{\theta(A(E)+t \vec{W}+l(0,0)) \theta(x \vec{U}+t \vec{W}+l(0,0))},
$$

$$
\begin{aligned}
& \omega(E)=\int_{E_{2 n+1}}^{E} \frac{z^{n}+b_{1} z^{n-1}+\ldots b_{n}}{2 \sqrt{\prod_{j=1}^{2 n+1}\left(z-E_{j}\right)}} d z, \quad \oint_{a_{k}} d \omega(E)=0 \quad(k=1, \ldots, n), \\
& (A(E))_{k}=\int_{\infty}^{E} \Omega_{k} \quad(k=1, \ldots, n),
\end{aligned}
$$

satisfies the Schrödinger equation with the potential (2.3.34). Here $\psi(x, t, E)$ and $\psi\left(x, t, E^{*}\right), E^{*}=T E$ (where $T$ is the involutory auto- 
morphism of $\Gamma$ interchanging the sheets) form a fundamental system of solutions of the Schrödinger equation.

However, the Riemann surface $\Gamma$ generates a series of trace formulae of another kind, namely linear trace formulae. They are obtained by integrating the form $E^{m} d \ln F$, where $F$ is defined by $(2.3 .35)$, over the boundary $\partial \widetilde{\Gamma}$ of $\widetilde{\Gamma}$, which is obtained by cutting $\Gamma$ along all the basic cycles $a_{i}$ and $b_{i}$, and they have the form

$$
\sum_{k=1}^{n} \gamma_{k}^{m}(x, t)=\sum_{k=1}^{n} \underset{a_{k}}{ } \sum^{m} \Omega_{k}-\underset{E=\infty}{\operatorname{res}}\left\{E^{m} d \ln F\right\}
$$

The evaluation of the residue in the first of these formulae has already been discussed and led to (2.3.34). For $m=2$ the evaluation of the residue and a subsequent differentiation with respect to $x$ give

$$
\frac{d}{d x} \sum_{k=1}^{n} \gamma_{k}^{2}(x, t)=\frac{u_{x x x}}{12}-\frac{u_{t}}{6} \text {. }
$$

It was observed by one of the present authors in [53] and [54] that a comparison between linear and non-linear trace formulae can be taken as a basis for integration of non-linear equations connected with Riemann surfaces.

This idea was used for the integration of the non-linear Schrodinger equation and the modified Korteweg-de Vries equation (see [52]).

When applied to the Korteweg-de Vries equation it is particularly effective: by differentiating the right-hand side of (A.3.2) and comparing the resulting identity with (A.3.4), we see that the function $u(x)$ (originally defined as the sum $u=-2 \Sigma \gamma_{i}+\Sigma E_{i}$ ) satisfies the K-dV equation, a calculation of the residue on the right-hand side of (A.3.3) for $m=1$ gives rise to an explicit representation for $u$. To integrate the higher order analogues of the $\mathrm{K}-\mathrm{dV}$ equation within this framework we must be justified in writing out explicitly the corresponding Jacobi problem, that is, selecting the corresponding direction (the vector $\vec{W}$ ) on the Jacobian of the Riemann surface. It turns out that the form of the corresponding vector $\vec{W}$ is uniquely determined by the condition that the right-hand sides of (A.3.3) after the evaluation of the residue and subsequent differentiation with respect to $x$, has the form $G u$, where $G$ is a linear differential operator with constant coefficients. Therefore, the form of the corresponding vectors $\vec{W}$ is determined by the asymptotic form of the expression $\frac{\varphi_{k}(E)}{\sqrt{R(E)}}$, as $E \rightarrow \infty$, where $\omega_{k}=\frac{\varphi_{k}(E)}{\sqrt{R(E)}} d E$. A detailed description of this method of integrating the higher order $\mathrm{K}-\mathrm{dV}$ equations is given in [54]. We quote here only the formula for $\vec{W}$ that describes the solution of the second $\mathrm{K}-\mathrm{dV}$ equation: 


$$
W_{j}=32 i\left\{c_{j_{3}}+\frac{1}{2} c c_{j_{2}}+c_{j_{1}}\left[\frac{3}{8} c^{2}-\frac{1}{2} \sum_{i>j} E_{i} E_{j}\right]\right\}, \quad c=\sum_{j=1}^{n} E_{j} .
$$

We recall that the second $\mathrm{K}-\mathrm{dV}$ equation has the form

$$
u_{t}=u_{x}^{(5)}-20 u_{x} u_{x x}-10 u u_{x x x}-30 u^{2} u_{x} \text {. }
$$

We ought to mention also that in this "trace" approach to the integration of non-linear equations we can use non-linear trace formulae, different in form and origin, which we call dynamic. The source of these formulae are the equations (2.4.3); for example, for the $\mathrm{K}-\mathrm{dV}$ equation it follows directly from (2.4.3) that

$$
\frac{\partial \gamma_{j}(x, t)}{\partial t}=2\left(u+2 \gamma_{j}\right) \frac{\partial \gamma_{j}}{\partial x},
$$

from which we obtain by summation over $j$ the equation

$$
\frac{d}{d x} \sum_{j=1}^{n} \gamma_{j}^{2}(x, t)=\frac{1}{2} u u_{x}-\frac{1}{4} u_{t}
$$

Comparing (A.3.5) and (A.3.4) we again confirm that $u$ is a solution of the $\mathrm{K}-\mathrm{dV}$ equation, and this argument makes no use of the Schrödinger equation, but deals all the time with objects directly connected with the Riemann surface.

Its has shown that when applied to hyperelliptic surfaces generated by a polynomial of even order $P(E)=\prod_{j=1}^{2 n}\left(E-E_{j}\right)$, this approach leads to the integration of a series of non-linear evolutionary systems, generalizing the non-linear Schrödinger equation and modified $\mathrm{K}-\mathrm{dV}$ equation, which were discussed above. Here are some examples of such systems:

$$
\begin{aligned}
& \left\{\begin{array}{l}
i u_{t}+u_{x x}-2 v u=0, \\
i v_{t}-v_{x x}+2\left(v \frac{u_{x}}{u}\right)_{x}=0,
\end{array}\right. \\
& \left\{\begin{array}{l}
v_{z}-6 v v_{x}+v_{x x x}-3\left[\frac{u_{x}}{u}\left(v_{x}-\frac{u_{x}}{u} v\right)\right]_{x}=0, \\
u_{z}-6 u_{x} v+u_{x x x}=0,
\end{array}\right. \\
& \left\{\begin{array}{l}
i f_{t}+f_{x x}-2 i f f_{x}-2 i v_{x}=0, \\
i v_{t}-v_{x x}-2 i(f v)_{x}=0,
\end{array}\right. \\
& \left\{\begin{array}{l}
v_{z}-6 v v_{x}+v_{x x x}-3\left[f\left(f v-i v_{x}\right)\right]_{x}=0, \\
f_{z}-3 f^{2} f_{x}+f_{x x x}-\frac{3}{2} i\left(f^{2}\right)_{x x}-6(v f)_{x}=0 .
\end{array}\right.
\end{aligned}
$$




\section{CONCLUDING REMARKS ${ }^{1}$}

1. In the summer of 1975 L. D. Faddeev brought to the attention of the authors new preprints which he had obtained in May in the USA. A paper of McKean and van Moerbeke "On Hill's equation" contains proofs of a number of the results quoted in Ch. 2, $\S 3$ of this survey. Apparently the authors of this preprint were not familiar with the papers [39], [42], [43], published in 1974. Incidentally, the text contains a historical inaccuracy: the fact that the Lamé potential $n(n+1) \delta^{\circ}(x)$ is finite-zoned was established not in 1975 but many years before (see, for example, our Introduction).

A number of preprints (of Kac and van Moerbeke) deals with periodic problems for the Toda chain and a discrete version of the K-dV equation. All these papers were written in 1975; the first, which contains only a small part of the results, has appeared in print recently (Proc. Nat. Acad. Sci. USA 72 (1975), 1627; Adv. in Math. 16 (1975), 160 ff.). In subsequent preprints some of the results of Ch. $3, \S 1$ of this survey, which have not previously been published, are obtained independently. The authors evidently are not familiar with the paper of Manakov [35] on discrete systems, in which an $L-A$ pair of operators for the discrete $\mathrm{K}-\mathrm{dV}$ equation was first found (see also Ch. $1, \S 5$ and Ch. $3, \S 1$ ).

In addition, we have received from Japan a preprint (by E. Date and S. Tanaka "Exact solutions for the periodic Toda lattice"), in which the method of the authors is also applied to the periodic problem for the Toda chain.

2. I. M. Gel'fand informs us that the survey of Gel'fand and Dikii "Asymptotic behaviour of the resolvent of Sturm-Liouville equations and the algebra of the Korteweg-de Vries equations", Uspekhi Mat. Nauk 30:5 (1975), 67-100 = Russian Math. Surveys 30:5 (1975), 77-113, contains the construction of the integrals for stationary problems for higher order $\mathrm{K}-\mathrm{dV}$ equations of which an account is given in Appendix 3 of this survey from Lax's recent preprint. They have found this construction independently and at the same time as Lax. Moreover, Gel'fand and Dikii also prove that these integrals are involutory, having subjected them to a concrete analysis (this result is not in Lax's paper). Without knowing the results of Gel'fand and Dikii, Novikov and Bogoyavlenskii have also proved that these integrals are involutory, as a consequence of a very simple and quite general theorem on the interrelation of the Hamiltonian formalisms of non-stationary and stationary equations. (Attention to this connection had already been drawn in [38], but it had not been formulated accurately.) We give here a statement of this theorem (see [64]). We recall that according to Gardner, Hamiltonian systems on a function space have the form

1 This addendum was received by the Editors on 24 September 1975. 


$$
\frac{\partial u}{\partial t}=\dot{u}=\frac{d}{d x} \frac{\delta I}{\delta u(x)}
$$

where

$$
I=\int P\left(u, u^{\prime}, u^{\prime \prime}, \ldots, u^{(n)}\right) d x, \quad u^{\prime}=\frac{\partial u}{\partial x}
$$

and $I$ is the Hamiltonian. We assume that $P$ does not depend explicitly on $x$. We consider the system formally without making precise the nature of the function space, which can even turn out to consist of increasing functions.

For the two functionals

$$
I=\int P d x, \quad J=\int Q d x
$$

the Poisson bracket has the form

$$
\langle I, J\rangle=\int\left(\frac{d}{d x} \frac{\delta J}{\delta u(x)}\right) \frac{\delta I}{\delta u(x)} d x,
$$

and the Hamiltonian $\langle I, J\rangle$ formally defines the commutator of the two flows (on any class of functions on a straight line). If the flows commute, $\langle I, J\rangle=0$, then we have, by definition,

$$
\left(\frac{d}{d x} \frac{\delta J}{\delta u(x)}\right) \frac{\delta I}{\delta u(x)}=\frac{d V(I, J)}{d x},
$$

where $V=V\left(u, u^{\prime}, \ldots\right)$. We consider the stationary problem $\frac{\delta I}{\delta u(x)}=0$ (or const) which has a Hamiltonian form in the canonical coordinates (see [38], Example 2 for $n=2$ )

$$
\left\{\begin{array}{l}
q_{1}=u, \quad q_{2}=u^{\prime}, \quad q_{3}=u^{\prime \prime}, \ldots, \quad q_{n}=u^{(n-1)} \\
p_{1}=\frac{\partial P}{\partial u^{\prime}}-\left(\frac{\partial P}{\partial u^{\prime \prime}}\right)^{\prime}+\left(\frac{\partial P}{\partial u^{\prime \prime \prime}}\right)^{\prime \prime}-\ldots \pm\left(\frac{\partial P}{\partial u^{(n)}}\right)^{(n-1)} \\
p_{2}=\frac{\partial P}{\partial u^{\prime \prime}}-\left(\frac{\partial P}{\partial u^{\prime \prime}}\right)^{\prime \prime}+\left(\frac{\partial P}{\partial u^{(4)}}\right)^{\prime \prime}-\ldots \pm\left(\frac{\partial P}{\partial u^{(n)}}\right)^{(n-2)} \\
\ldots \ldots \ldots \ldots \ldots \ldots \ldots \ldots \ldots \ldots \ldots \ldots \ldots \ldots \ldots \\
p_{n}=\frac{\partial P}{\partial u^{(n)}}
\end{array}\right.
$$

with the Hamiltonian

$$
H(p, q)=P-u^{\prime} p_{1}-u^{\prime \prime} p_{2}-\ldots-u^{(n !} p_{n},
$$

provided that they can all be expressed by the coordinates $(p, q)$. This is in text-books on the calculus of variations with higher order derivatives.

$$
\text { If } I=\int P\left(u, u^{\prime}, \ldots, u^{(n)}\right) d x, J=\int Q\left(u, u^{\prime}, \ldots, u^{(m)}\right) d x, \text { and }\langle I, J\rangle=0
$$

then on the set of fixed points of the flow (1) the functional $J$ defines the flow $\dot{u}=\frac{d}{d x} \frac{\delta J}{\delta u(x)}$ as a finite-dimensional dynamical system, for example, 
as a higher order $\mathrm{K}-\mathrm{dV}$ equation in [38]. Under these conditions we have the following general and fairly simple result:

THEOREM. If $m\left\langle n\right.$ and $\langle I, J\rangle=0$, then the flow $\dot{u}=\frac{d}{d x} \frac{\delta J}{\delta u(x)}$ on the set of fixed points of the flow (1) is a one-parameter family of finitedimensional dynamical systems, depending on the constant of integration $\frac{\delta I}{\delta u(x)}=d ;$ all these systems are Hamiltonian and are given by the Hamiltonian $V_{d}(I, J)$, which can be expressed in terms of the phase coordinates (3), where

$$
\frac{d}{d x} V_{d}(I, J)=\left(\frac{d}{d x} \frac{\delta J}{\delta u(x)}\right)\left(\frac{\delta I}{\delta u(x)}-\cdots d\right)
$$

Example: let $J=\int \frac{u^{2}}{2} d x$; in this case the flow $\dot{u}=\frac{d}{d x} \frac{\delta J}{\delta u(x)}=u^{\prime}$ defines the group of shifts through $x$ and commutes with $I$ if $P$ does not depend explicitly on $x$. Then we have

$$
V_{0}(I, J)=H(p, q), \quad \frac{d V}{d x}=u^{\prime} \frac{\delta I}{\delta u(x)},
$$

where $H(p, q)$ is a Hamiltonian of the stationary problem for the flow (1).

From the theorem it follows, obviously, that if two integrals $J_{1}$ and $J_{2}$ of the flow (1) commute in the non-stationary problem, $\left\langle J_{1}, J_{2}\right\rangle=0$, then their images $V\left(I, J_{1}\right)$ and $V\left(I, J_{2}\right)$ commute in the phase space of the stationary problem with the canonical coordinates (3).

The application of the theorem to the theory of $\mathrm{K}-\mathrm{dV}$ equations is obvious, because in this theory we have many commuting flows in a function space (the higher $\mathrm{K}-\mathrm{dV}$ equations according to Gardner); the images of these flows on the set of fixed points of any one, as stated in [38], give a set of commuting systems in the stationary problem. It is interesting that in [38] a complete set of integrals $J_{1}, \ldots, J_{n}$ is found for the stationary problem, which can be expressed explicitly in terms of the spectrum of the Sturm-Liouville operator (Hill, Schrödinger). In a later paper we shall show how to express these integrals of Novikov in terms of the integrals of Lax-Gel'fand-Dikii and vice versa (see [65]).

3. Very recently Krichever, developing a technique of Dubrovin and Its, and Matveev, has found a beautiful algebraic-geometric method of constructing analogues of finite-zone solutions of the periodic problem (in $x$ ) for "two-dimensional K-dV" equation or the equations of KadomtsevPetviashviki (see [37] and Ch. 1, $\$ 2$ of this survey) and some other equations of Zakharov and Shabat, containing an additional coordinate $y$. It is remarkable that in the construction of solutions of "two-dimensional K-dV" equation by Krichever's scheme an arbitrary Riemann surface of genus $g$ appears. The possibility of applying this method to problems of 
algebraic geometry (and of constructing explicit universal fiberings over the variety of moduli of Riemann surfaces) by the scheme of NovikovDubrovin (see [43] and Ch. 2, §4 of this survey and also Ch. 3, §2) is here subjected to a careful analysis.

4. Very recently a new paper of Moser has appeared (Three integrable Hamiltonian systems connected with isospectral deformations, Adv. in Math. 16 (1975), $354 \mathrm{ff}$. ), in which he finds an $L-A$ pair for a very interesting class of discrete systems of classical particles on a straight line with various interaction potentials. (According to work of Calogero and Sutherland, in some of these models it is known that the corresponding quantum problem is completely soluble). An interesting group theoretical approach to the systems of Moser and Calogero has now been developed by Perelomov and Ol'shanetskii ("Complete integrable systems connected with Lie algebras"). These systems lead, in contrast to the Toda chain, to "non-local" operators $L$ and cannot be described by the methods of the present survey, even when the periodic problem is meaningful.

\section{References}

[1] G. Borg, Eine Umkehrung der Sturm-Liouvilleschen Eigenwertaufgabe, Acta Math. 78 (1946), 1-96. MR 7--382.

[2] V. Bargmann, Determination of a central field of force from the elastic scattering phase shifts, Phys. Rev. 75 (1949), 301-303.

[3] I. M. Gel'fand and B. M. Levitan, On the determination of a differential equation by its spectral function, Izv. Akad. Nauk SSSR Ser. Mat. 15 (1951), 309-360. MR 13-558.

[4] V. A. Marchenko, Some problems of the theory of one-dimensional differential operators. I, Trudy Moskov. Mat. Obshch. 1 (1952), 327-420. MR 15-315.

[5] L. D. Faddeev, Properties of the $S$-matrix of the one-dimensional Schrödinger equation, Trudy Mat. Inst. Steklov 73 (1964), 314-336. MR 31 \# 2446.

[6] I. V. Stankevich, On an inverse problem of spectral analysis for the Hill equation, Dokl. Akad. Nauk SSSR 192 (1970), 34-37. MR 41 \# 7195.

= Soviet Math. Dokl. 11 (1970), 582-586.

[7] E. L. Ince, Further investigations into the periodic Lamé functions, Proc. Roy. Soc. Edinburgh 60 (1940), 83-99. MR 2-46.

[8] I. Kay and H. Moses, Reflectionless transmission through dielectrics and scattering potentials, J. Appl. Phys. 27 (1956), 1503-1508.

[9] H. Hochstadt, On the determination of a Hill's equation from its spectrum, Arch. Rational Mech. and Anal. 19 (1965), 535-562. MR 31 \#6019.

[10] N. I. Akhiezer, A continuous analogue to orthogonal polynomials on a system of intervals, Dokl. Akad. Nauk SSSR 141 (1961), 263-266. MR 25 \# 4383.

= Soviet Math. Dokl. 2 (1961), 1409-1412.

[11] A. B. Shabat, On potentials with zero reflection coefficient, in the coll. Dinamika sploshnoi sredy (Dynamics in a dense medium), No. 5, 130-145, Novosibirsk 1970.

[12] K. M. Case and M. Kac, A discrete version of the inverse scattering problem, J. Mathematical Phys. 14 (1973), 594-603. MR 48 \# 10392.

[13] I. M. Gel'fand and B. M. Levitan, On a simple identity for the eigenvalues of a SturmLiouville operator, Uspekhi Mat. Nauk 30:6 (1975). 
[14] L. A. Dikii, On a formula of Gel'fand-Levitan, Uspekhi Mat. Nauk 8:2 (1953), 119-123. MR 15-130.

[15] E. C. Titchmarsh, Eigenfunction expansions associated with second-order differential equations, Vol.2, Ch. XXI, Clarendon Press, Oxford 1958. MR 20 \# 1065.

Translation: Razlozheniya po sobstvennym funktsiyam, svyazannye s differentsial'nymi uravneniyami vtorogo poryadka, Izdat. Inost. Lit., Moscow 1961.

[16] G. B. Whitham, Non-linear dispersive waves, Proc. Roy. Soc. Ser. A283 (1965), 238-261. MR 31 \# 996.

[17] M. D. Kruskal and N. Zabusky, Interaction of "solitons" in a collisionless plasma and the recurrence of initial states, Phys. Rev. Lett. 15 (1965), 240-243.

[18] C. S. Gardner, J. M. Green, M. D. Kruskal, and R. M. Miura, Method for solving the Korteweg-de Vries equation, Phys. Rev. Lett. 19 (1967), 1095-1098.

[19] P. D. Lax, Integrals of non-linear equations of evolution and solitary waves, Comm. Pure Appl. Math. 21 (1968), 467-490. MR 38 \# 3620.

= Matematika 13:5 (1969), 128-150.

[20] V. E. Zakharov, A kinetic equations for solitons, Zh. Eksper. Teoret. Fiz. 60 (1971), 993-1000.

= Soviet Physics JETP 33 (1971), 538-541.

[21] V. E. Zakharov and L. D. Faddeev, The Korteweg-de Vries equation is a completely integrable Hamiltonian system, Funktsional. Anal. i Prilozen. 5:4 (1971), 18-27. MR 46 \# 2270.

= Functional Anal. Appl. 5 (1971), 18-27.

[22] C. S. Gardner, Korteweg-de Vries equation and generalizations. IV, The Korteweg-de Vries equation as a Hamiltonian system, J. Mathematical Phys. 12 (1971), 1548-1551. MR 44 \# 3615.

[23] V. E. Zakharov, On the problem of stochestization of one-dimensional chains of nonlinear oscillators, Zh. Eksper. Teoret. Fiz. 65 (1973) 219-225. = Soviet Physics JETP 38 (1974), 108-110.

[24] V. S. Dryuma, On an analytic solution of the two-dimensional K-dV equation, Letters to Zh. Eksper. Teoret. Fiz. 19:12 (1974), 753-755.

[25] V. E. Zakharov and A. B. Shabat, Exact theory of two-dimensional self-focusing and one-dimensional self-modulation of waves in non-linear media, Zh. Eksper. Teoret. Fiz. 61 (1971), 118-134.

= Soviet Physics JETP 34 (1972), 62-69.

[26] V. E. Zakharov and A. B. Shabat, Interaction between solitons in a stable medium, Zh. Eksper. Teoret. Fiz. 64 (1973), 1627-1639.

= Soviet Physics JETP 37 (1973), 823-828.

[27] V. E. Zakharov and S. V. Manakov, On a resonance interaction of wave packets in nonlinear media, Letters to Zh. Eksper. Teoret. Fiz. 18:7 (1973), 413-417.

[28] L. A. Takhtadzhyan, Exact theory of propagation of ultrashort optical impulses in two-level media, Zh. Eksper. Teoret. Fiz. 66 (1974) 476-489.

= Soviet Physics JETP 39 (1974), 228-233.

[29] M. J. Ablowitz, D. J. Kaup, A. C. Newell and H. Segur, Method for solving the sineGordon equation, Phys. Rev. Lett. 30 (1973), 1262-1264.

[30] V. E. Zakharov, L. A. Takhtadzhyan and L. D. Faddeev, Complete description of solutions of the "sine-Gordon" equation, Dokl. Akad. Nauk SSSR 219 (1974), 1334-1337.

= Soviet Physics Dokl. 19 (1975), 824-826. 
[31] M. Toda, Waves in non-linear lattice, Progr. Theoret. Phys. Suppl. 45 (1970), 174-200.

[32] M. Henon, Integrals of the Toda lattice, Phys. Rev. B9 (1974), 1921-1923.

[33] H. Flaschka, Toda lattice. I, Phys. Rev. B9 (1974), 1924-1925.

[34] H. Flaschka, Toda lattice. II, Progr. Theoret. Phys. 51 (1974), 703-716.

[35] S. V. Manakov, On the complete integrability and stochastization in discrete dynamical systems, Zh. Eksper. Teoret. Fiz. 67 (1974) 543-555.

[36] A. B. Shabat, On the Korteweg-de Vries equation, Dokl. Akad. Nauk SSSR 211 (1973), 1310-1313. MR 48 \# 9137.

= Soviet Math. Dokl. 14 (1973), 1266-1270.

[37] V. E. Zakharov and A. B. Shabat, A scheme for the integration of non-linear equations of mathematical physics by the method of inverse scattering theory. I, Funktsional. Anal. i Prilozin. 8:3 (1974), 43-53.

[38] S. P. Novikov, The periodic Korteweg-de Vries problem I. Funktsional. Anal. i Prilozhen. $8: 3(1974), 54-66$.

[39] S. P. Novikov and B. A. Dubrovin, The periodic problem for the Korteweg-de Vries and the Sturm-Liouville equations, Petrovskii Seminar 6 March 1974, Uspekhi Mat. Nauk 29:6 (1974), 196-197.

[40] B. A. Dubrovin, The inverse problem of scattering theory for periodic finite-zone potentials, Funktsional. Anal. i Prilozhen. 9:1 (1975), 65-66.

[41] A. R. Its and V. B. Matveev, On Hill operators with finitely many lacunae, Funktsional. Anal. i Prilozhen. 9:1 (1975), 69-70.

[42] B. A. Dubrovin and S. P. Novikov, Periodic and conditionally periodic analogues of multisoliton solutions of the Korteweg-de Vries equation, Zh. Eksper. Teoret. Fiz. 67 (1974), 2131-2143.

= Soviet Physics JETP 40 (1974), 1058-1063.

[43] B. A. Dubrovin and S. P. Novikov, The periodic problem for the Korteweg-de Vries and Sturm-Liouville equations. Their connection with algebraic geometry. Dokl. Akad. Nauk SSSR 219 (1974), 19-22.

[44] V. A. Marchenko, The periodic Korteweg-de Vries problem, Dokl. Akad. Nauk SSSR 217 (1974), 276-279. MR 50 \# 7855.

[45] V. A. Marchenko, The periodic Korteweg-de Vries problem, Mat. Sb. 95 (1974), $331-356$.

[46] B. V. Dubrovin, The periodic problem for the Korteweg-de Vries equation in the class of finite-zone potentials, Funktsional. Anal. i Prilozin. 9:3 (1975).

[47] A. R. Its and V. B. Matveev, Hill operators with finitely many lacunae and multisoliton solutions of the Korteweg-de Vries equation, Trudy Mat. Fiz. 23 (1975), 51-67.

[48] E. A. Kuznetsov and A. B. Mikhailov, The stability of stationary waves in non-linear media with weak dispersion, Zh. Eksper. Teoret. Fiz. 67 (1974), 1717-1727.

= Soviet Physics JETP 40 (1974), 855-859.

[49] I. M. Krichever, Non-reflecting potentials against the background of finite-zone potentials, Funktsional. Anal. i Prilozhen. 9:2 (1975).

[50] P. D. Lax, Periodic solutions of the K-dV equation, Lectures in Appl. Math. 15 (1974), 85-96.

[51] B. A. Dubrovin, Finite-zone linear operators and Abelian varieties, Uspekhi Mat. Nauk 31:4 (1976),

[52] A. R. Its, Canonical systems with a finite-zone spectrum and periodic solutions of the non-linear Schrödinger equation, Vestnik Leningrad Univ. Mat. 1976.

[53] V. B. Màtveev, A new method of integrating the Korteweg-de Vries equation, Uspekhi Mat. Nauk 30:6 (1975), 201-203. 
[54] A. R. Its and V. B. Matveev, On a class of solutions of the K-dV equation, in Problemy matematicheskoi fiziki (Problems of mathematical physics), No 8, Leningrad University 1976.

[55] G. Springer, Introduction to Riemann surfaces, Addison-Wesley, New York 1957. Translation: Vvedenie v teoriyu riemanovykh poverkhnostei, Izdat. Inost. Lit., Moscow 1961.

[56] E. I. Zverovich, Boundary problems of the theory of analytic functions Uspekhi Mat. Nauk 26:1 (1971), 113-180.

$=$ Russian Math. Surveys 26:1 (1971), 117-192.

[57] V. V. Golubev, Lektsii po integrirovaniyu uravnenii dvizheniya tyazhelogo tverdogo tela s odnoi nepodvizhnoi tochkoi, Gostekhizdat, Moscow 1953. MR 15-904.

Translation: Lectures on the integration of the equations of motion of a heavy rigid body about a fixed point, Nat. Sci. Foundation, Israel Program Sci. Transl. MR 22 \# 7298.

[58] W. Goldberg, On the determination of a Hill's equation from its spectrum, Bull. Amer. Math. Soc. 80 (1974), 1111-1112.

[59] E. I. Dinaburg and Ya. G. Sinai, On the spectrum of the one-dimensional Schrödinger equation with quasi-periodic potential, Funktsional. Anal. i Prilozhen. 9 (1975), 8-21.

[60] V.P. Kotlyarov, The periodic problem for the non-linear Schrödinger equation, in Matematicheskaya fizika i funktsionalnyi analiz, (Mathematical physics and functional analysis), Trudy FTINT Akad. Nauk Ukrain. SSR 6 (1975).

[61] V. A. Kozel, On a class of solutions of the sine-Gordon equation, in Matematicheskaya fizika i funkstionalnyu analiz (Mathematical physics and functional analysis), Trudy FTINT Akad. Nauk Ukrain. SSR 6 (1975).

[62] I. M. Gel'fand and L. A. Dikii, Asymptotic behaviour of the resolvent of Sturm-Liouville equations and the algebra of the Korteweg-de Vries equations, Uspekhi Mat. Nauk 30:5 (1975), 67-100.

$=$ Russian Math. Surveys 30:5 (1975), 77-113.

[63] P. D. Lax, Periodic solutions of the K-dV equations, Comm. Pure Appl. Math. 28 (1975), $141-188$.

[64] O. I. Bogoyavlenskii and S. P. Novikov, On the connection between the Hamiltonian formalisms of stationary and non-stationary problems, Funktsional Anal. i Prilozhen. 10:1 (1976).

[65] O. I. Bogoyavlenskii, On integrals of higher order stationary equations and the eigenvalues of Hill operators, Funktsional. Anal. i Prilozhen. 10:2 (1976).

[66] V. E. Zakharov and S. V. Manakov, On the complete integrability of the non-linear Schrödinger equation, TMF 19 (1974).

Received by the Editors 2 June 1975

Translated by G. and R. Hudson 